\title{
Photometric survey, modelling, and scaling of long-period and low-amplitude asteroids ${ }^{\star}$
}

\author{
A. Marciniak ${ }^{1}$, P. Bartczak ${ }^{1}$, T. Müller ${ }^{2}$, J. J. Sanabria ${ }^{3}$, V. Alí-Lagoa ${ }^{2}$, P. Antonini ${ }^{4}$, R. Behrend ${ }^{5}$, L. Bernasconi ${ }^{6}$, \\ M. Bronikowska ${ }^{7}$, M. Butkiewicz-Bạk ${ }^{1}$, A. Cikota $^{8}$, R. Crippa $^{9}$, R. Ditteon ${ }^{10}$, G. Dudziński ${ }^{1}$, R. Duffard ${ }^{11}$, \\ K. Dziadura ${ }^{1}$, S. Fauvaud ${ }^{12}$, S. Geier ${ }^{3}, 13$, R. Hirsch ${ }^{1}$, J. Horbowicz ${ }^{1}$, M. Hren ${ }^{8}$, L. Jerosimic ${ }^{8}$, K. Kamiński ${ }^{1}$, \\ P. Kankiewicz ${ }^{14}$, I. Konstanciak ${ }^{1}$, P. Korlevic ${ }^{8}$, E. Kosturkiewicz ${ }^{1}$, V. Kudak ${ }^{15}, 16$, F. Manzini ${ }^{9}$, N. Morales ${ }^{11}$,
} M. Murawiecka ${ }^{17}$, W. Ogłoza ${ }^{18}$, D. Oszkiewicz ${ }^{1}$, F. Pilcher ${ }^{19}$, T. Polakis ${ }^{20}$, R. Poncy ${ }^{21}$, T. Santana-Ros ${ }^{1}$, M. Siwak ${ }^{18}$, B. Skiff ${ }^{22}$, K. Sobkowiak ${ }^{1}$, R. Stoss ${ }^{8}$, M. Żejmo ${ }^{23}$, and K. Żukowski ${ }^{1}$

1 Astronomical Observatory Institute, Faculty of Physics, A. Mickiewicz University, Słoneczna 36, 60-286 Poznań, Poland e-mail: am@amu.edu.pl

2 Max-Planck-Institut für Extraterrestrische Physik, Giessenbachstrasse 1, 85748 Garching, Germany

3 Instituto de Astrofísica de Canarias, C/vía Lactea s/n, 38205 La Laguna, Tenerife, Spain

${ }^{4}$ Observatoire des Hauts Patys, 84410 Bédoin, France

5 Geneva Observatory, 1290 Sauverny, Switzerland

${ }^{6}$ Les Engarouines Observatory, 84570 Mallemort-du-Comtat, France

7 Institute of Geology, A. Mickiewicz University, Krygowskiego 12, 61-606 Poznań, Poland

8 OAM - Mallorca, Camí de l'Observatori s/n, 07144 Costitx Mallorca, Illes Balears, Spain

9 Stazione Astronomica di Sozzago, 28060 Sozzago, Italy

10 Rose-Hulman Institute of Technology, CM 1715500 Wabash Ave., Terre Haute, IN 47803, USA

11 Departamento de Sistema Solar, Instituto de Astrofísica de Andalucía (CSIC), Glorieta de la Astronomía s/n, 18008 Granada, Spain

12 Observatoíre du Bois de Bardon, 16110 Taponnat, France

13 Gran Telescopio Canarias (GRANTECAN), Cuesta de San José s/n, 38712 Breña Baja, La Palma, Spain

14 Astrophysics Division, Institute of Physics, Jan Kochanowski University, Świętokrzyska 15, 25-406 Kielce, Poland

15 Institute of Physics, Faculty of Natural Sciences, University of P. J. Šafárik, Park Angelinum 9, 04001 Košice, Slovakia

16 Laboratory of Space Researches, Uzhhorod National University, Daleka st. 2a, 88000 Uzhhorod, Ukraine

17 NaXys, Department of Mathematics, University of Namur, 8 Rempart de la Vierge, 5000 Namur, Belgium

18 Mt. Suhora Observatory, Pedagogical University, Podchorążych 2, 30-084 Cracow, Poland

194438 Organ Mesa Loop, Las Cruces, NM 88011, USA

${ }^{20}$ Command Module Observatory, $121 \mathrm{~W}$. Alameda Dr., Tempe, AZ 85282, USA

${ }^{21}$ Rue des Écoles 2, 34920 Le Crès, France

22 Lowell Observatory, 1400 West Mars Hill Road, Flagstaff, 86001 Arizona, USA

23 Kepler Institute of Astronomy, University of Zielona Góra, Lubuska 2, 65-265 Zielona Góra, Poland

Received 30 June 2017 / Accepted 11 September 2017

\section{ABSTRACT}

Context. The available set of spin and shape modelled asteroids is strongly biased against slowly rotating targets and those with low lightcurve amplitudes. This is due to the observing selection effects. As a consequence, the current picture of asteroid spin axis distribution, rotation rates, radiometric properties, or aspects related to the object's internal structure might be affected too.

Aims. To counteract these selection effects, we are running a photometric campaign of a large sample of main belt asteroids omitted in most previous studies. Using least chi-squared fitting we determined synodic rotation periods and verified previous determinations. When a dataset for a given target was sufficiently large and varied, we performed spin and shape modelling with two different methods to compare their performance.

Methods. We used the convex inversion method and the non-convex SAGE algorithm, applied on the same datasets of dense lightcurves. Both methods search for the lowest deviations between observed and modelled lightcurves, though using different approaches. Unlike convex inversion, the SAGE method allows for the existence of valleys and indentations on the shapes based only on lightcurves.

Results. We obtain detailed spin and shape models for the first five targets of our sample: (159) Aemilia, (227) Philosophia, (329) Svea, (478) Tergeste, and (487) Venetia. When compared to stellar occultation chords, our models obtained an absolute size scale and major topographic features of the shape models were also confirmed. When applied to thermophysical modelling (TPM), they provided a very good fit to the infrared data and allowed their size, albedo, and thermal inertia to be determined.

Conclusions. Convex and non-convex shape models provide comparable fits to lightcurves. However, some non-convex models fit notably better to stellar occultation chords and to infrared data in sophisticated thermophysical modelling (TPM). In some cases TPM showed strong preference for one of the spin and shape solutions. Also, we confirmed that slowly rotating asteroids tend to have higher-than-average values of thermal inertia, which might be caused by properties of the surface layers underlying the skin depth.

Key words. techniques: photometric - minor planets, asteroids: general

\footnotetext{
* The photometric data is only available at the CDS via anonymous ftp to cdsarc.u-strasbg.fr (130.79.128.5) or via http://cdsarc.u-strasbg.fr/viz-bin/qcat?J/A+A/610/A7
} 


\section{Introduction}

Physical parameters of asteroids such as the period of rotation and orientation of the spin axis are related to various processes that these bodies undergo. The rotation of large asteroids probably reflects the primordial spin acquired during the accretion phase in the protoplanetary disc (Johansen \& Lacerda 2010), which for smaller objects was later modified by impacts, collisions, and thermal forces, which are strongest for small asteroids (Bottke et al. 2006). Asteroid rotations can reveal both their internal cohesion and the degree of fragmentation (Holsapple 2007). Numerical simulations by Takeda \& Ohtsuki (2009) suggest that bodies of a rubble-pile structure usually spin down as a result of impacting events. Also, the long-term evolution under the thermal reradiation force (YORP effect) can both spin up and spin down asteroids (Rubincam 2000). However, so far only the spin-up of the rotation period has been directly detected (e.g. Lowry et al. 2007, 2014; Kaasalainen et al. 2007; Durech et al. 2008).

The spatial distribution of asteroid spin axes suggests that the largest bodies generally preserved their primordial, prograde spin, while smaller ones, with diameters less than $30 \mathrm{~km}$, seem to be strongly affected by the YORP effect that pushes these axes towards extreme values of obliquities (Hanuš et al. 2013). The spins of prograde rotators under the YORP effect influence can be captured into spin-orbit resonances, sometimes even forming spin clusters (Slivan 2002; Kryszczyńska et al. 2012).

However, what is now known about these physical properties of asteroids is based on statistically non-representative samples. Most of the well-studied asteroids (those with the spin and shape model) are targets of relatively fast spin and substantial elongation of shape, possibly also coupled with extreme spin axis obliquity, which results in fast and large brightness variations (Fig. 1). The reason for this state are the observing selection effects discussed in our first paper on this subject (Marciniak et al. 2015, hereafter M2015), and summarised in the next section.

Asteroid shape models created by lightcurve inversion methods are naturally most detailed when created basing on rich datasets of dense lightcurves. High-quality lightcurves from at least five apparitions gained over a wide range of aspect and phase angles are a necessary prerequisite to obtain unique spin and shape solutions with main topographic features (usually coming in pairs of two indistinguishable mirror solutions for the pole). The obtained models can be convex representations of real shapes (in the convex inversion method by Kaasalainen \& Torppa 2001; Kaasalainen et al. 2001), but can also be non-convex, more closely reproducing real asteroid shapes when supported by auxiliary data (in KOALA and ADAM algorithms, Carry et al. 2012; Viikinkoski et al. 2015), but also based on lightcurves alone (in the SAGE algorithm, Bartczak et al. 2014; Bartczak \& Dudziński 2017).

Even after the Gaia Solar System catalogue is released, which is expected at the beginning of the next decade, the most reliable way to study spins (sidereal periods and spin axis positions) of a number of new bodies of low amplitudes and long periods is the traditional dense photometry performed on a network of small and medium-sized ground-based telescopes. The precise shape modelling technique is, and will most probably remain, the only tool allowing a substantial number of such challenging targets to be studied in detail because Gaia and most of the other sky surveys will deliver only a few tens of sparse datapoints for each observed asteroid, only providing ellipsoidal approximations of the real shapes. However, the number of targets with precise shape models cannot be as large as when modelling

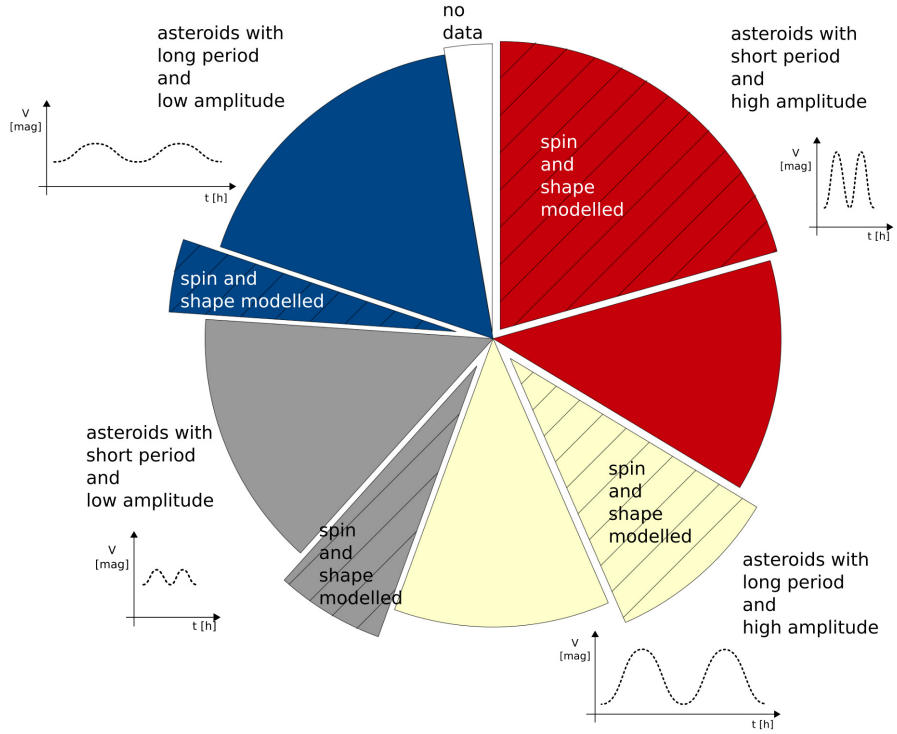

Fig. 1. Current distribution of known periods and maximum amplitudes among the $\sim 1200$ brightest main belt asteroids (based on LCDB, Warner et al. 2009, updated 2016 September 5 ). Division values are $P=12 \mathrm{~h}$ and $a_{\max }=0.25 \mathrm{mag}$. The amount of spin and shape modelled targets is marked within each group. Asteroids with specific features are over-represented, while others are largely omitted.

on sparse data because of the high demand of observing time, which reaches hundreds of hours for each long-period target (see Table A.1).

Detailed asteroid shape models with concavities are in high demand for precise density determinations (Carry 2012), modelling the thermal YORP and Yarkovsky effects (Vokrouhlický et al. 2015) - including self-heating - and accurate thermophysical modelling (Delbo et al. 2015) from which one can infer their sizes, albedos, surface roughness, and thermal inertia values, allowing further studies of their composition and surface and subsurface properties. Apart from studying asteroid parameters for themselves, such research has other very practical applications. Large asteroids are very good calibration standards for infrared observatories like Herschel, APEX, and ALMA, perfectly filling the gap in the flux levels of stellar and planetary calibration sources (Müller \& Lagerros 2002; Müller et al. 2014a). However, their flux changes have to be clearly predictable, and should not vary much over short timescales. Slowly rotating asteroids of low lightcurve amplitudes are best for such applications.

In this work we perform spin and shape modelling using two lightcure inversion methods: the convex inversion method (Kaasalainen \& Torppa 2001; Kaasalainen et al. 2001) and the non-convex SAGE algorithm (Bartczak et al. 2014; Bartczak \& Dudziński 2017). Later we validate and at the same time compare the resulting shapes by fitting them to data from other techniques: multi-chord stellar occultations, and all available thermal infrared data. This way our shape models also get absolute size scale, both radiometric and non-radiometric.

The next section discusses the selection effects in asteroid studies, and briefly describes our observing campaign to counteract them. Section 3 describes spin and shape modelling methods, and brings a description of thermophysical modelling and occultation fitting procedures used primarily to scale our models. Section 4 contains the observing campaign intermediate results, another set of targets with corrected period determinations. In Sect. 5 we present models for five targets of our sample that 


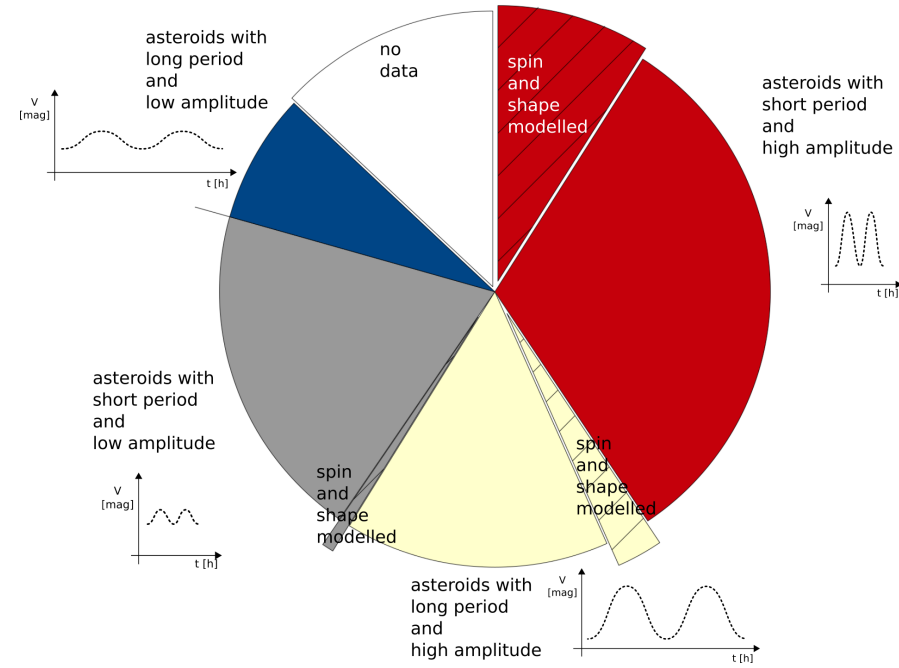

Fig. 2. Same as Fig. 1, but for the $\sim 2270$ fainter MB targets, with $H$ between 11 and $13 \mathrm{mag}$ (source: LCDB). There are 270 large-amplitude targets (from those on the right side of the chart) with available spin and shape model, while only a few low-amplitude targets with a model (left side). Judging from the sample of only those small asteroids that have available shape models, and not taking into consideration the distribution of the amplitudes of all asteroids, can create a false impression that almost all small asteroids are strongly elongated.

have enough data for full spin and shape modelling, scale them by thermophysical modelling, and where possible also by occultations. The last section describes the conclusions and planned future work. Appendix A contains observation details and new lightcurves.

\section{Selection effects and the observing campaign}

\subsection{Observing and modelling biases in asteroid studies}

Statistical considerations in this section are based on the Minor Planer Center Lightcurve Database (LCDB, Warner et al. 2009, updated 2016 September 5) using a sample of the $~ 1200$ brightest main belt Asteroids (those with absolute magnitudes $H \leq$ 11 mag, Fig. 1) ${ }^{1}$, which translates to diameters down to 12-37 km, depending on albedo (after MPC conversion table) $)^{2}$. The rationale behind such a choice is that in this sample $97 \%$ the main belt bodies have rotation period determined and available information on the lightcurve amplitude from at least one apparition. Among the fainter targets ( $H$ between 11 and 13 mag) there are many bodies with no information on the rotation parameters, so one cannot draw firm conclusions on the median period or amplitude. However, the selection effects discussed here are even more profound in the group of these fainter targets (equivalent diameters from 37 to $5 \mathrm{~km}$, Fig. 2).

Because asteroid modelling using lightcurve inversion requires data from a wide variety of observing geometries, it is far more observationally demanding to gather a sufficient number of dense lightcurves over multiple apparitions for long-period targets (here those with $P \geq 12 \mathrm{~h}$ ) than for those with quicker rotation. However, not including them in spin and shape studies means omitting around half of the whole asteroid population

\footnotetext{
1 The exact number of asteroids with certain $H$ magnitude varies over time, due to updates in magnitude and albedo determinations gathered in LCDB.

2 http://www . minorplanetcenter. net/iau/lists/Sizes. html
}

in question (see the upper left and lower right part of Fig. 1). Moreover, recent results from Kepler-K2 continuous observations spanning weeks show that there are substantially more slow-rotators among faint main belt asteroids and Jupiter Trojans than ground-based studies have shown (Szabó et al. 2016, 2017; Molnár et al. 2017). Observations from the ground are naturally burdened with selection bias, absent when observing for long time spans from space.

Another problematic group of asteroids are those with low amplitudes of their brightness variations (here those with $a_{\max } \leq$ $0.25 \mathrm{mag}$ ). They are almost as numerous as those with large amplitudes (greater than $0.25 \mathrm{mag}$ ); even so, they are spin and shape modelled very rarely (see the left part of Fig. 1) because their study requires photometric data of very good accuracy, while data most often used for modelling asteroids nowadays come as a byproduct of large astrometric surveys. As such, these data are characterised by very low photometric accuracy (0.1-0.2 mag on average, Hanuš et al. 2011), so the modelling is missing most of the low-amplitude population (Ďurech et al. 2016).

As a result there is a large "white spot" in the parameter space, where very little is known about large groups of asteroids (upper left part of Fig. 1). We do not know their spin axis distribution, their shapes, or internal structure. Some of them may be tumbling, can be tidally despun by a large companion, or slowed down by the YORP effect. Also, their thermal inertia might be different than those rotating faster, as it seems to increase with the rotation period (Harris \& Drube 2016) due to sampling of different depths that have different thermal properties. However, for now only $10 \%$ of the asteroids observed in the infrared by IRAS and WISE space observatories have thermal inertia determined. It has been stressed that efforts should be made to carry out sophisticated thermophysical modelling of slowly rotating asteroids. Thermophysical modelling (TPM) techniques work best for objects with reliable shape and spin information. The existing multi-epoch, multi-wavelength thermal measurements can then be used to determine radiometric properties (effective size, geometric albedo, thermal inertia, surface roughness, emissivity) and to study if a given shape and spin solution can explain all measurements simultaneously (see e.g. Müller et al. 2014b).

\subsection{Observing campaign}

In order to counteract the above-mentioned selection effects, we are conducting an extensive and long-term observing campaign targeting around a hundred bright $(H \leq 11 \mathrm{mag})$ main belt asteroids that display both a long period of rotation $(P \geq 12 \mathrm{~h})$ and a low lightcurve amplitude $\left(a_{\max } \leq 0.25 \mathrm{mag}\right)$, which are the objects that have been largely omitted in most of the previous spin and shape studies. We coordinate the multi-site campaign with about 20 observing stations placed around the world, from Europe through western US, to Korea and Japan. The detailed description of the campaign can be found in M2015. Table 1 gives the information on the observing sites participating in this project. It also includes chosen sites of the group led by R. Behrend as we use some of the archival data gathered by this group, so far published only on the Observatoire de Genève website ${ }^{3}$.

We perform unfiltered, or $R$-filter photometric observations of a given target until we get full rotation coverage and possibly also register notable phase angle effects. After that, the observations within one apparition are folded together in a composite lightcurve (Figs. A.1-A.24) for synodic period determination. When the period is found to be in disagreement with the value

\footnotetext{
http://obswww. unige.ch/ behrend/page_cou.html
} 
Table 1. Observing sites participating in this project.

\begin{tabular}{|c|c|c|c|c|}
\hline Site name & Abbreviation & IAU code & Location & Telescope \\
\hline Borowiec Observatory (Poland) & Bor. & 187 & $52 \mathrm{~N}, 17 \mathrm{E}$ & $0.4 \mathrm{~m}$ \\
\hline Montsec Observatory (Catalonia, Spain) & OAdM & C65 & $42 \mathrm{~N}, 01 \mathrm{E}$ & $0.8 \mathrm{~m}$ \\
\hline Organ Mesa Observatory (NM, USA) & Organ M. & G50 & $32 \mathrm{~N}, 107 \mathrm{~W}$ & $0.35 \mathrm{~m}$ \\
\hline Winer Observatory (AZ, USA) & Winer & 648 & $32 \mathrm{~N}, 111 \mathrm{~W}$ & $0.70 \mathrm{~m}$ \\
\hline Bisei Spaceguard Center (Okayama, Japan) & Bisei & 300 & $35 \mathrm{~N}, 134 \mathrm{E}$ & $0.5 \mathrm{~m}$ and $1 \mathrm{~m}$ \\
\hline Mt. Suhora Astronomical Observatory (Poland) & Suh. & & $50 \mathrm{~N}, 20 \mathrm{E}$ & $0.25 \mathrm{~m}$ and $0.60 \mathrm{~m}$ \\
\hline Le Bois de Bardon Observatory (France) & Bardon & & $45 \mathrm{~N}, 0 \mathrm{E}$ & $0.28 \mathrm{~m}$ \\
\hline Adiyaman Observatory (Turkey) & Adi. & & $38 \mathrm{~N}, 38 \mathrm{E}$ & $0.6 \mathrm{~m}$ \\
\hline Derenivka Observatory (Ukraine) & Der. & K99 & $48 \mathrm{~N}, 22 \mathrm{E}$ & $0.4 \mathrm{~m}$ \\
\hline JKU Astronomical Observatory, Kielce (Poland) & Kie. & B02 & $51 \mathrm{~N}, 21 \mathrm{E}$ & $0.35 \mathrm{~m}$ \\
\hline Pic du Midi Observatory (France) & Pic. & 586 & $43 \mathrm{~N}, 0 \mathrm{E}$ & $0.6 \mathrm{~m}$ \\
\hline Teide Observatory (Tenerife, Spain) & Teide & 954 & $28 \mathrm{~N}, 16 \mathrm{~W}$ & $0.8 \mathrm{~m}$ \\
\hline Roque de los Muchachos (La Palma, Spain) & ORM & 950 & $29 \mathrm{~N}, 18 \mathrm{~W}$ & $1 \mathrm{~m}$ and $1.2 \mathrm{~m}$ \\
\hline Kitt Peak National Observatory (AZ, USA) & KPNO & G82 & $32 \mathrm{~N}, 112 \mathrm{~W}$ & $1 \mathrm{~m}$ \\
\hline Lowell Observatory (AZ, USA) & Lowell & 688 & $35 \mathrm{~N}, 112 \mathrm{~W}$ & $0.78 \mathrm{~m}$ \\
\hline Command Module Observatory, Tempe (AZ, USA) & Tempe & V02 & $33 \mathrm{~N}, 112 \mathrm{~W}$ & $0.32 \mathrm{~m}$ \\
\hline Cerro Tololo Interamerican Observatory (Chile) & CTIO & 807 & $30 \mathrm{~S}, 71 \mathrm{~W}$ & $0.6 \mathrm{~m}$ \\
\hline La Sagra Observatory (Spain) & La Sagra & & $38 \mathrm{~N}, 3 \mathrm{~W}$ & $0.35 \mathrm{~m}$ \\
\hline Piszkesteto Mountain Station (Hungary) & Pisz. & 461 & $48 \mathrm{~N}, 20 \mathrm{E}$ & $1 \mathrm{~m}$ \\
\hline Sobaeksan Optical Astronomy Observatory (Korea) & Sobaek & 345 & $37 \mathrm{~N}, 128 \mathrm{E}$ & $0.61 \mathrm{~m}$ \\
\hline Flarestar Observatory (Malta) & Flare. & 171 & $36 \mathrm{~N}, 14 \mathrm{E}$ & $0.25 \mathrm{~m}$ \\
\hline Astronomy Observatory of Sertao de Itaparica (Brazil) & OASI & Y28 & $9 \mathrm{~S}, 39 \mathrm{~W}$ & $1 \mathrm{~m}$ \\
\hline Observatoire des Engarouines (France) & Engar. & A14 & $44 \mathrm{~N}, 5 \mathrm{E}$ & $0.21 \mathrm{~m}$ \\
\hline Le Crès (France) & Le Cres & 177 & $44 \mathrm{~N}, 4 \mathrm{E}$ & $0.4 \mathrm{~m}$ \\
\hline Observatoire des Hauts Patys, Bédoin (France) & Hauts Patys & 132 & $44 \mathrm{~N}, 5 \mathrm{E}$ & $0.30 \mathrm{~m}$ \\
\hline OAM - Mallorca (Spain) & OAM & 620 & $40 \mathrm{~N}, 3 \mathrm{E}$ & $0.3 \mathrm{~m}$ \\
\hline Stazione Astronomica di Sozzago (Italy) & Sozzago & A12 & $45 \mathrm{~N}, 9 \mathrm{E}$ & $0.40 \mathrm{~m}$ \\
\hline
\end{tabular}

in the MPC Lightcurve Database (LCDB), the observations concentrate on this target to confirm the new period value. The observations are repeated in each apparition until data of good quality and quantity from at least five well-spaced apparitions are gathered, including those already available in the literature. In the course of the campaign the maximum amplitudes of some targets appeared to be larger than $0.25 \mathrm{mag}$, while periods of some others were shorter than $12 \mathrm{~h}$, violating our initial selection criteria, nonetheless they remained on our target list.

Table A.1 summarises new observations for 11 targets studied in this paper ( 6 targets with corrected periods, and 5 with new models), presenting values important for spin and shape studies: mid-date of given lightcurve, sky ecliptic longitude of the target $(\lambda)$, phase angle $(\alpha)$, observing run duration, photometric error, and the observer's name with the observing site.

The best way to present the trustability of period determinations and the reliability of the obtained spin and shape models is to present the quality and quantity of supporting lightcurves and the model fit. Our data are presented in Appendix A in the form of composite lightcurves. Alongside lightcurves of modelled targets, we present the orientation on the zero phase of the best shape model, generated using the ISAM service ${ }^{4}$, described in Marciniak et al. (2012). In Figs. 13, 16, 18, 22, and 24 we also present model example fits to lightcurves.

\section{Spin and shape modelling; scaling the models}

\subsection{Lightcurve inversion methods}

The Shaping Asteroids with Genetic Evolution (SAGE) modelling algorithm was developed at the Astronomical Observatory Institute of AMU Poznań (Bartczak et al. 2014; Bartczak \& Dudziński 2017). Thus, we utilise the local cluster with the SAGE code for the spin and shape modelling in

\footnotetext{
4 http://isam.astro.amu.edu.pl
}

parallel with the now classical convex inversion method by Kaasalainen \& Torppa (2001), Kaasalainen et al. (2001).

SAGE is a genetic algorithm that mutates the shape models to find the specimens that are best suited to lightcurve data. Although main belt asteroids can only be observed at relatively small phase angles (up to $30^{\circ}$ at most), it has been shown that their lightcurves contain signatures of non-convex topographic features, so that these features can be successfully reproduced in the shape models (Bartczak \& Dudziński 2017). When modelling on lightcurves is a priori complemented by auxiliary data like adaptive optics or occultation contours in one multi-data inversion process, such non-concavities gain more support (as in models created using ADAM algorithm, Viikinkoski et al. 2015; Hanuš et al. 2017). However, when SAGE non-convex models based exclusively on lightcurves are a posteriori compared to multi-chord occultations, their topographic features are confirmed, as has been shown in the case of binary asteroid (90) Antiope (Bartczak et al. 2014), but also in simulations and real-case studies performed recently by Bartczak \& Dudziński (2017).

The modelling here was performed independently using the convex inversion and SAGE methods, on the same datasets, taking as a starting value only the synodic period estimates from a set of composite lightcurves. The solutions for the poles and the shapes were searched over the whole possible range. From each method a set of internally consistent spin and shape solutions was obtained, and the uncertainty on the spin parameters was evaluated from the scatter of the best solutions for the pole (taking all the solutions with the best root mean square deviation (RMSD) enlarged by up to $10 \%$ ). The lightcurves produced by models from both methods fit the data around the noise level without big differences in the overall quality of the fit (measured by RMSD) between the two methods, so it might seem that the models fit the lightcurves in the same way. However, the overall sum of deviations does not reflect the subtle differences of the 

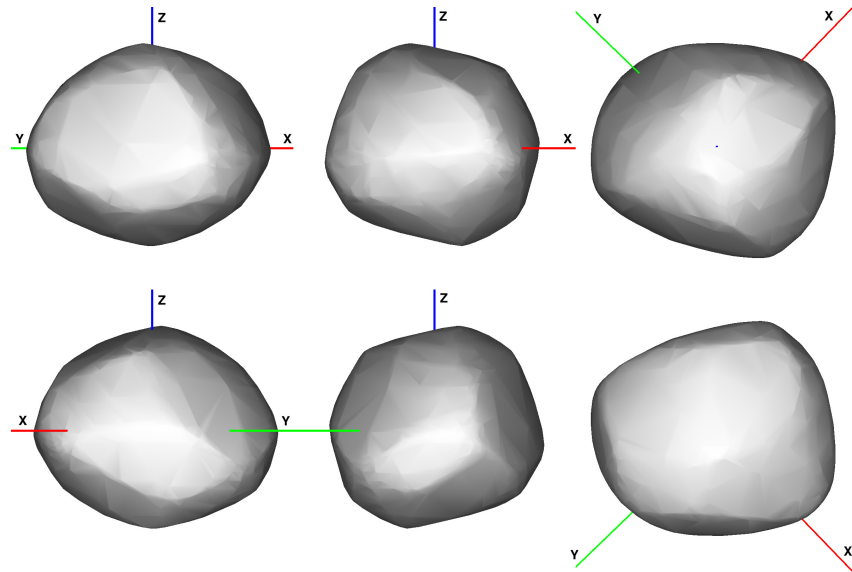

Fig. 3. Convex shape model of (159) Aemilia from the lightcurve inversion method shown in six projections. The $z$-axis is the axis of rotation Compare with Fig. 4.
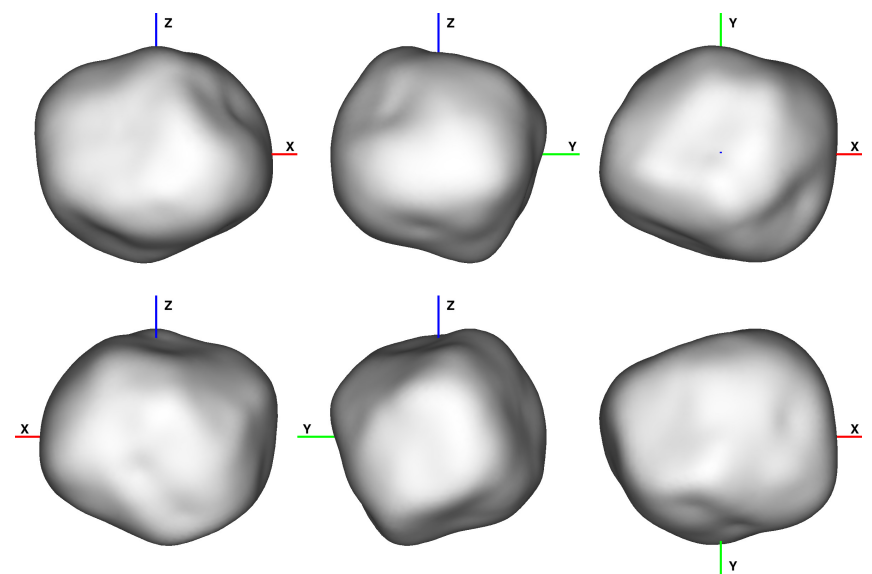

Fig. 4. Non-convex shape model of (159) Aemilia from the SAGE algorithm shown in six projections. The $z$-axis is the axis of rotation, while the $x$-axis is the longest axis of the shape model.

lightcurve fits between the two methods, like sometimes visible better fitting of the SAGE models to critical features (e.g. deep minima or abrupt dimmings), where non-convex features most clearly manifest themselves. Such features, due to their short duration, usually contain far fewer datapoints than other lightcurve fragments, so their influence on the RMSD value is very small. However during the SAGE optimisation process the biggest weight is given to the worst fitting lightcurves, so in further iterations these fragments have a bigger influence on the shape model and are fitted better. Still, the final (unweighted) RMSD value might be the same, when other lightcurves have a slightly worse fit, and the large number of points in them makes the small change more significant for RMSD. So, using only the RMSD of the fit, we have no means to tell which model best represents the real shape. Here we present one of possible solutions for the shape chosen from a family of very similar shape models; however, without a method to estimate shape uncertainties, it is hard to compare the performance of the two methods.

The shape models from the two methods were often similar to each other, clearly indicating that convex models are the convex hulls of more complex shapes, successfully reproduced by the SAGE algorithm. However, in some cases the shapes looked distinctively different, and only the pole-on projections were similar. The orientation of the two models in pairs of Figs. 3 and 4 is the same, so these shape projections can be directly compared. Different positions of the $x$ - and $y$-axes are caused by their different definitions: in SAGE models the rotation axis is the axis of biggest inertia, and the $x$-axis of the smallest inertia. In convex models, the $z$-axis should also correspond to the biggest inertia, but the $x$-axis is connected with the epoch of the first observation, so its orientation does not correspond to any specific feature of the shape model $^{5}$.

\subsection{Thermophysical modelling}

This radiometric technique consists in the exploitation of thermal data in the mid- to far-infrared and data in the visible. Thermophysical models allow the derivation of size, albedo, and thermal properties for small bodies (see Delbo et al. 2015, and references therein). There are different model implementations available, ranging from simple thermal models assuming spherical shapes at opposition without heat conduction into the surface to more sophisticated thermophysical model implementations which take complex shapes and rotational properties into account; at the same time heat conduction, shadowing effects, and self-heating effects are calculated for a given illumination and observing geometry. Here, we are interested in assigning reliable scales to the obtained spin-shape solutions, deriving high-quality geometric albedos, estimating the surface's thermal inertia, and finding indications for the levels of surface roughness. For our analysis, we therefore used a TPM code developed by Lagerros (1996, 1997, 1998) and extensively tested and validated (e.g. by Müller \& Lagerros 1998, 2002). The TPM allows the use of all kind of shape solutions (convex and non-convex). It considers the true observing and illumination geometry to calculate the surface temperature distribution for any given epoch. The $1 \mathrm{D}$ heat conduction into the surface, shadowing, and self-heating effects are calculated. Good examples for TPM applications to main belt asteroids can be found in Müller et al. (2014a) for Ceres, Pallas, Vesta, and Lutetia, or in Marsset et al. (2017) for Hebe.

We applied the following procedure:

- we use a given convex or non-convex shape-spin solution (see previous section);

- the small-scale surface roughness is approximated by hemispherical segment craters covering a smooth surface. We consider different levels of roughness ranging from 0.1 to 0.9 for the rms of the surface slopes;

- the thermal inertia is considered as a free parameter, with values between zero (i.e. no heat conductivity, surface is in instantaneous equilibrium with the insolation) and $2000 \mathrm{Jm}^{-2} \mathrm{~K}^{-1} \mathrm{~s}^{-1 / 2}$ (bare rock surface with very high heat conductivity);

- the characterisation of the reflected light is given by the H-G (or H-G1-G2) solutions;

- for each observed and calibrated infrared measurement we determine all possible size and albedo solutions for the full range of thermal inertias and roughness levels;

- we search for the lowest $\chi^{2}$ solution in size, albedo, and thermal inertia/roughness for all thermal IR measurements combined;

- we calculate the $3 \sigma$ solutions for the available set of thermal measurements: we consider $1 /(N-v)$, where $N$ is the number of (thermal) measurements and $v$ is the number of

5 There is a different sequence of rotations in the reference frame definitions of the convex and non-convex models, so if both models were to be placed in the plane of sky, the rotation of $-270^{\circ}$ around the $z$-axis would be necessary for the SAGE models to match the orientations of the convex models. 
free parameters, here $v=2$ because we fit for diameter and thermal inertia. We also fit for albedo, but here we make use of another measurement (the $H$ magnitude). We define the $n-\sigma$ confidence interval by accepting all solutions that have

$\chi^{2}<\chi_{\min }^{2}+n^{2}$

where $\chi^{2}$ is the actual

$$
\chi^{2}=\sum\left(\frac{\mathrm{obs}-\mathrm{mod}}{e r r}\right)^{2} .
$$

- solutions are only accepted if the reduced $\chi^{2}$ values are reasonably close to 1.0 . In this case the "unreduced" $\chi^{2}$ will have a minimum equal to $N-2$, and the $3 \sigma$ limit for $N$ observations is at $N-2+3^{2}=N-7$;

- the minima for the reduced $\chi^{2}$ for each shape and spin solution are given in Table 6.

The results of this procedure are the following:

- we find the best radiometric size which corresponds to the size of an equal-volume sphere and can be used to scale the given shape-spin solution;

- we determine the geometric albedo (closely connected to the given $H$ magnitude);

- we estimate the possible range of thermal inertias (higher or lower values would introduce problems when comparing pre- and post-opposition IR data);

- assuming low roughness gives lower values for the thermal inertia, higher levels of roughness lead to slightly higher thermal inertias. Our IR data are usually not good enough to break the degeneracy between thermal inertia and roughness, but we consider this aspect in the solutions in Table 7;

- in some cases the minimum $\chi^{2}$ values for the different shapespin solutions for a given target are very different: in these cases we favour the solution with the best $\chi^{2}$ fit.

The radiometric technique is not very sensitive to the exact shape, and provides sizes and albedos with around 5\% accuracy in the most favourable cases. It is the most productive way of determining sizes and albedos for large samples of asteroid IR measurements (as coming from IRAS, AKARI, WISE surveys), but it also allows spin properties to be constrained and wide ranges of shape-spin solutions to be discarded. The radiometric analysis uses thermal data from different epochs, phase angles, wavelengths, and rotational phases. The resulting radiometric size is therefore closely related to the full 3D body, while occultations are only representative of the 2D cross section of the body.

\subsection{Stellar occultation fitting}

Stellar occultations by main belt asteroids are being observed by a few active groups (like Noth American ${ }^{6}$, European ${ }^{7}$, or East Asian observers $\left.{ }^{8}\right)$, and published in the Planetary Data System ${ }^{9}$ (PDS; see Dunham et al. 2016), providing great complementary data for asteroid physical studies. Occultation timing measurements of such events enable scaling of the otherwise scale-free shape models, and also confirm their major and intermediate-size

\footnotetext{
6 http://WwW . asteroidoccultation.com/observations/ Results/

7 http://www . euraster. net/results/index.html

8 http://sendaiuchukan.jp/data/occult-e/occult-e.html

9 http://sbn.psi.edu/pds/resource/occ.html
}

topographic features. Very often they can also break the mirrorpole symmetry intrinsic to the lightcurve inversion models.

When the occultation observation is successful and at least three well-spaced chords are obtained with good accuracy, it is possible to overlay the occultation shadow chords and the photometric asteroid model (as in e.g. Timerson et al. 2009; Durech et al. 2011) with relatively small uncertainty regarding the exact position of the model contour.

Of the five targets modelled here, these multichord events were available for two of them and it allowed us to independently scale, compare, and verify their spin and shape models. The translation of the timings from PDS to chords on the Earth fundamental plane $(\xi, \eta)$ has been done using the method described in Durech et al. (2011). Both convex and non-convex 3D shape models obtained here have been translated into scalable 2D contours, according to sky-plane shape orientation for a given moment, and then overlaid on the timing chords so as to minimise the overall rms deviations between the contour and the chords, taking into account the timing uncertainties. As a result, the models were scaled in kilometres with good accuracy; the maximum size of a given shape model was later translated into the diameter of the equivalent volume sphere. Results are described and plotted in Sect. 4. The list of all the observers of asteroid occultations that were utilised in this work can be found in Appendix B (Dunham et al. 2016).

\section{Corrected period determinations}

The first and rather unexpected result of our observing campaign was that as much as $25 \%$ of the numerous bright main belt asteroids with both long period and small amplitude had a previously incorrectly determined synodic period of rotation (M2015). Their period quality codes in LCDB were 3, 2+, and 2. Although periods with code 2 and lower should be considered unreliable; usually, all period values with codes higher than $1+$ are taken into account in the majority of spin state studies of asteroids. The wrong period determination in the cases that we studied was due to previous incomplete or noisy lightcurve coverage, which often led the alias period to be incorrectly identified as the true rotation period.

As an example, in Figs. A.1-A.5 we present a few more cases where we found rotation periods substantially different from the values accepted in LCDB (Warner et al. 2009). Below, we briefly review previous works on these targets and describe our findings. Their previous and new period values are presented in Table 2. Together with targets for which we already had corrected period values (M2015, and Marciniak et al. 2016), their overall number (16) compared to the number of our targets for which we found secure period determinations (65) confirms our previous findings that around a quarter of bright long-period asteroids with low amplitudes had incorrectly determined rotation periods. More precisely, out of 16 targets with incorrect periods, four targets had period quality code 3 , two had code $2+$, and ten had code 2 . So if only the reliable periods (code 3 and $2+$ ) were considered, the percentage of incorrect values in the group of bright long-period, low-amplitude targets would be around $10 \%$.

\section{1. (551) Ortrud}

The first report on lightcurve and period of (551) Ortrud was made by Robinson (2002), who determined a $13.05 \mathrm{~h}$ period based on an asymmetric, bimodal lightcurve from the year 2001. Although three consecutive works on this target, Behrend et al. (2014) in 2003 and 2006, and Buchheim (2007) in 2006 reported 
A. Marciniak et al.: Long-period and low-amplitude asteroids

Table 2. Synodic periods and amplitude values found within this project compared to literature data gathered previously in LCDB.

\begin{tabular}{lcccc}
\hline \hline Asteroid name & $\begin{array}{c}\text { Amplitude (LCDB } \\
\text { and this work) } \\
{[\mathrm{mag}]}\end{array}$ & $\begin{array}{c}\text { Period (LCDB) } \\
{[\mathrm{h}]}\end{array}$ & $\begin{array}{c}\text { Period } \\
\text { quality } \\
\text { code }\end{array}$ & $\begin{array}{c}\text { Period (this work) } \\
{[\mathrm{h}]}\end{array}$ \\
\hline Targets with new periods: & $0.14-0.19$ & 13.05 & 2 & $\mathbf{1 7 . 4 1 6} \pm 0.001$ \\
(551) Ortrud & $0.07-0.20$ & 16.54 & 2 & $\mathbf{2 4 . 9 8 7} \pm 0.007$ \\
(581) Tauntonia & $0.15-0.42$ & 39.0 & 2 & $\mathbf{1 6 9 . 5 2} \pm 0.06$ \\
(830) Petropolitana & $0.16-0.28$ & 19.746 & 2 & $\mathbf{2 9 . 7 1} \pm 0.04$ \\
(923) Herluga & $0.20-0.24$ & 39.1 & $2+$ & $\mathbf{7 8 . 4 4} \pm 0.01$ \\
(932) Hooveria & $0.06-0.20$ & 14.612 & $2+$ & $\mathbf{1 1 . 1 9 8} \pm 0.002$ \\
(995) Sternberga & & & & \\
\hline Targets with models: & $0.17-0.26$ & 24.476 & 3 & $24.486 \pm 0.002$ \\
(159) Aemilia & $0.06-0.20$ & 52.98 & $2 \mathrm{~A}$ & $\mathbf{2 6 . 4 6 8} \pm 0.003$ \\
(227) Philosophia & $0.09-0.24$ & 22.778 & $2+$ & $22.777 \pm 0.005$ \\
(329) Svea & $0.15-0.30$ & 16.104 & $2+$ & $16.105 \pm 0.002$ \\
(478) Tergeste & $0.03-0.30$ & 13.34 & 3 & $13.342 \pm 0.002$ \\
(487) Venetia & & & & \\
\hline
\end{tabular}

Notes. Boldface indicates period determinations substantially differing from previously accepted values.

a different period $(17.59,17.401$, and $17.416 \mathrm{~h}$, respectively), the adopted value in LCDB remained unchanged due to the low quality code assigned to these determinations.

During our observations, we found that only the period of $17.420 \pm 0.001 \mathrm{~h}$ can fit the data we gathered in 2016 (Fig. A.1), confirming the findings from the three latter works. So it turned out that the correct period has already been identified, but our data put it on firmer ground. The amplitude was at the level of $0.19 \pm 0.01 \mathrm{mag}$. The lightcurve, as in each observed apparition, is characterised by narrow minima and wide complex maxima.

\section{2. (581) Tauntonia}

Previously observed by group led by R. Behrend in 2005 and 2006, Tauntonia displayed very low amplitude lightcurves that seemed to fit a period of around 16.5-16.2 h (Behrend et al. 2014). Stephens (2010) found instead that the period was $24.90 \mathrm{~h}$, based on an asymmetric $0.20 \mathrm{mag}$ amplitude lightcurve from the year 2010 .

Our data from 2016 can be best folded with period $24.987 \pm$ $0.007 \mathrm{~h}$, creating an unusual though consistent composite lightcurve (Fig. A.2), and $0.18 \pm 0.02$ mag amplitude, confirming the determination by Stephens (2010).

\section{3. (830) Petropolitana}

The only lightcurve observations of Petropolitana were reported by Behrend et al. (2014), with a period estimated to $39.0 \mathrm{~h}$, based only on three separate fragments. In Hanuš et al. (2016), there is a model of this target based exclusively on sparse data from astrometric sky surveys, where the sidereal period is $37.347 \mathrm{~h}$, found by scanning a standard period span of up to $100 \mathrm{~h}$.

Our observations suggest a much longer period: $169.52 \pm$ $0.06 \mathrm{~h}$, based on calibrated data with nightly zero point adjustments (Fig. A.3). The lightcurve behaviour is bimodal with a large amplitude $(0.42 \pm 0.02 \mathrm{mag})$. So this is a very long-period target, but not low-amplitude.

\section{4. (923) Herluga}

The only previous work on the lightcurve of (923) Herluga was published by Brinsfield (2009). The period determined at that time, $19.746 \mathrm{~h}$, was based on an imperfect composite lightcurve with some clearly misfitting fragments.

Our observations of this target did not allow us to find a satisfactory fit to any period until 2016, when we gathered 11 long lightcurve fragments. The only period that fits the new data (and data from all the previous observations) is $29.71 \pm 0.04 \mathrm{~h}$, which applied to the data from the year 2016 reveals a complex, trimodal lightcurve where one of the minima is deeper than the others (Fig. A.4). The amplitude was unusually large for this target: $0.28 \pm 0.02 \mathrm{mag}$.

\section{5. (932) Hooveria}

The first period determinations for Hooveria, 29.947 or $30.370 \mathrm{~h}$, were made by Sada (2004) from a bimodal folded lightcurve behaviour. Another set of data was obtained by Warner et al. (2010) and a period of $39.15 \mathrm{~h}$ was found, producing a monomodal lightcurve of rather large amplitude for this type (0.22 mag). In the same work, Warner et al. (2010) reanalysed the data obtained by Sada (2004) and was also able to fit them with a $39.15 \mathrm{~h}$ period, now making it monomodal.

Our extensive observations of Hooveria in late 2016 and careful nightly zero point adjustments using CMC15, APASS, and Gaia catalogue stars have shown that the rotation period of Hooveria must be twice as long, being $78.44 \pm 0.01 \mathrm{~h}$ and producing a bimodal lightcurve with clearly asymmetric extrema and $0.24 \pm 0.01$ mag amplitude (Fig. A.5). Fitting these data with a $39 \mathrm{~h}$ period would require large shifts in reduced magnitudes of steps bigger than $0.05 \mathrm{mag}$, much larger than the absolutisation errors.

\section{6. (995) Sternberga}

All of the previous reports on the period of (995) Sternberga claimed different values: Barucci et al. (1992) give $16.406 \mathrm{~h}$; Behrend et al. (2014) estimated $P>12 \mathrm{~h}$; Stephens (2005) found $15.26 \mathrm{~h}$, later corrected to $14.612 \mathrm{~h}$ in Stephens (2013) based on new data of larger amplitude.

Our analysis of this target since the beginning suggests that none of the previous values can be confirmed, and instead the period is either 22.404 or $11.202 \mathrm{~h}$ (Marciniak et al. 2014). Finally, data from the apparition in 2016 confirmed the lower 
Table 3. Parameters of the spin models of the five targets studied here, and the uncertainty values.

\begin{tabular}{|c|c|c|c|c|c|c|c|c|c|}
\hline \multirow{2}{*}{$\begin{array}{r}\text { Sidereal } \\
\text { period [hours] }\end{array}$} & \multicolumn{2}{|c|}{ Pole 1} & \multicolumn{2}{|c|}{ Pole 2} & \multirow{2}{*}{$\begin{array}{c}\text { RMSD } \\
\text { [mag] }\end{array}$} & \multirow{2}{*}{$\begin{array}{l}\text { Observing span } \\
\text { (years) }\end{array}$} & \multirow[t]{2}{*}{$N_{\text {app }}$} & \multirow[t]{2}{*}{$N_{\mathrm{lc}}$} & \multirow[t]{2}{*}{ Method } \\
\hline & $\lambda_{p}$ & $\beta_{p}$ & $\lambda_{p}$ & $\beta_{p}$ & & & & & \\
\hline \multicolumn{10}{|l|}{ (159) Aemilia } \\
\hline 24.4787 & $139^{\circ}$ & $+68^{\circ}$ & $348^{\circ}$ & $+59^{\circ}$ & 0.014 & $1981-2015$ & 6 & 45 & convex LI \\
\hline \pm 0.0001 & $\pm 18^{\circ}$ & $\pm 8^{\circ}$ & $\pm 18^{\circ}$ & $\pm 6^{\circ}$ & & & & & \\
\hline 24.4787 & $139^{\circ}$ & $+66^{\circ}$ & $349^{\circ}$ & $+63^{\circ}$ & 0.014 & " & " & $\prime \prime$ & SAGE \\
\hline \pm 0.0001 & $\pm 7^{\circ}$ & $\pm 5^{\circ}$ & $\pm 7^{\circ}$ & $\pm 6^{\circ}$ & & & & & \\
\hline \multicolumn{10}{|l|}{ (227) Philosophia } \\
\hline 26.4614 & $95^{\circ}$ & $+19^{\circ}$ & $272^{\circ}$ & $-1^{\circ}$ & 0.011 & 2006-2016 & 5 & 97 & convex LI \\
\hline \pm 0.0001 & $\pm 5^{\circ}$ & $\pm 4^{\circ}$ & $\pm 6^{\circ}$ & $\pm 2^{\circ}$ & & & & & \\
\hline 26.4612 & $97^{\circ}$ & $+16^{\circ}$ & $271^{\circ}$ & $0^{\circ}$ & 0.009 & " & " & $\prime \prime$ & SAGE \\
\hline \pm 0.0003 & $\pm 5^{\circ}$ & $\pm 5^{\circ}$ & $\pm 5^{\circ}$ & $\pm 5^{\circ}$ & & & & & \\
\hline \multicolumn{10}{|l|}{ (329) Svea } \\
\hline 22.7670 & $33^{\circ}$ & $+51^{\circ}$ & - & - & 0.010 & 1986-2016 & 6 & 60 & convex LI \\
\hline \pm 0.0001 & $\pm 15^{\circ}$ & $\pm 10^{\circ}$ & - & - & & & & & \\
\hline 22.7671 & $21^{\circ}$ & $+47^{\circ}$ & - & - & 0.011 & " & " & $\prime \prime$ & SAGE \\
\hline \pm 0.0002 & $\pm 7^{\circ}$ & $\pm 5^{\circ}$ & - & - & & & & & \\
\hline \multicolumn{10}{|l|}{ (478) Tergeste } \\
\hline 16.10308 & $2^{\circ}$ & $-42^{\circ}$ & $216^{\circ}$ & $-56^{\circ}$ & 0.011 & 1980-2016 & 6 & 48 & convex LI \\
\hline \pm 0.00003 & $\pm 2^{\circ}$ & $\pm 3^{\circ}$ & $\pm 6^{\circ}$ & $\pm 4^{\circ}$ & & & & & \\
\hline 16.10312 & $4^{\circ}$ & $-43^{\circ}$ & $218^{\circ}$ & $-56^{\circ}$ & 0.011 & " & " & $\prime \prime$ & SAGE \\
\hline \pm 0.00003 & $\pm 6^{\circ}$ & $\pm 5^{\circ}$ & $\pm 9^{\circ}$ & $\pm 7^{\circ}$ & & & & & \\
\hline \multicolumn{10}{|l|}{ (487) Venetia } \\
\hline 13.34133 & $78^{\circ}$ & $+3^{\circ}$ & $252^{\circ}$ & $+3^{\circ}$ & 0.012 & 1984-2015 & 8 & 34 & convex LI \\
\hline \pm 0.00001 & $\pm 7^{\circ}$ & $\pm 10^{\circ}$ & $\pm 8^{\circ}$ & $\pm 12^{\circ}$ & & & & & \\
\hline 13.34133 & $70^{\circ}$ & $+8^{\circ}$ & $255^{\circ}$ & $+8^{\circ}$ & 0.011 & " & " & $\prime \prime$ & SAGE \\
\hline \pm 0.00002 & $\pm 6^{\circ}$ & $\pm 11^{\circ}$ & $\pm 5^{\circ}$ & $\pm 10^{\circ}$ & & & & & \\
\hline
\end{tabular}

Notes. Column 1 gives the sidereal period of rotation; Cols. 2-5 give two sets of pole J2000.0 longitude and latitude; Col. 6 gives the rms deviations of the model lightcurves from the data; Cols. 7-9 give the photometric dataset parameters (observing span, number of apparitions, and individual lightcurve fragments). The last column contains the name of the lightcurve inversion (LI) method. The preferred pole solutions are shown in bold. The second pole solution of (329) Svea, though possible in the lightcurve inversion, was clearly rejected by occultation fitting.

value providing a good fit to $11.198 \pm 0.002 \mathrm{~h}$; this period was unambiguously found in spite of a very small amplitude of $0.06 \pm 0.01 \mathrm{mag}$ (Fig. A.6). Also, it fits all the previously obtained data.

In summary, the substantial number of periods that needed a revision was found among the brightest main belt targets $(H \leq 11)$ available to most small telescopes. Among the fainter targets these effects can be expected to an even greater extent, due to more noise in the photometric data. So one has to be careful when interpreting, for example a frequency-diameter plot, especially in the regions where fainter targets reside (diameters less than $\sim 30 \mathrm{~km}$ ). Many such targets might have incorrect period values, but a huge number of them are simply not present in the plot because their periods are unknown. Those that are present in the small diameter range of the frequency-diameter plot are strongly influenced by observing biases, favouring large amplitudes and short periods.

From our campaign, since the beginning of the project in 2013, we have gathered around $8000 \mathrm{~h}$ of photometric data, resulting in a few tens of full composite lightcurves of our long-period, low-amplitude targets each year. This dataset enables spin and shape modelling of the first representatives of our sample.

\section{Individual models}

In the following we provide the description of previous works on given target and the new data obtained within this work, presented as composite lightcurves in Figs. A.7-A.24. Next we describe the modelling process and the results of the spin and shape solutions presented in Table 3 and pairs of figures (see Figs. 3 and 4). Table 3 gives the spin solutions from both methods with uncertainty and RMSD (root mean square deviation) values. The first column gives the sidereal period value, the next four columns give two pairs of solutions for the north pole of the spin axis (J2000 ecliptic coordinates), all with uncertainty values. In the fifth column there is the observing span in years, number of apparitions $\left(N_{\text {app }}\right)$, and individual lightcurves $\left(N_{\text {lc }}\right)$ used to create the models. The last column provides the code of the modelling method. Tables 4 and 5 give the values for the diameters from the occultation fitting, and Table 7 the diameters from thermophysical modelling, both techniques described in the following sections. Additionally, Table 7 gives the best fitting albedo and thermal inertia values. For reference, the effective diameters from IRAS (Tedesco et al. 2004), AKARI (oney W.), and WISE (Mainzer et al. 2011; Masiero et al. 2011) surveys are given. 
Table 4. Equivalent volume sphere diameters of (159) Aemilia models fitted to the occultation from 2 May 2009.

\begin{tabular}{ccc}
\hline \hline & Pole 1 & Pole 2 \\
\hline CONVEX & $130 \pm 7 \mathrm{~km}$ & $130 \pm 8 \mathrm{~km}$ \\
SAGE & $135 \pm 7 \mathrm{~km}$ & $138 \pm 7 \mathrm{~km}$ \\
\hline
\end{tabular}

Notes. Compare with radiometric diameter from TPM in Table 7.

Table 5. Equivalent volume sphere diameters of the (329) Svea models pole 1, fitted to two occultations: from 28 December 2011 and 7 March 2013.

\begin{tabular}{ccc}
\hline \hline & 2011 & 2013 \\
\hline CONVEX & $72 \pm 4 \mathrm{~km}$ & $74 \pm 5 \mathrm{~km}$ \\
SAGE & $70 \pm 4 \mathrm{~km}$ & $72 \pm 3 \mathrm{~km}$ \\
\hline
\end{tabular}

Notes. Compare with radiometric diameter from TPM in Table 7.

A model example fit to the lightcurves is presented in Figs. 13, 16, and others. Additionally, to visualise what combination of aspect and shape can produce the given lightcurves, next to the composite lightcurves in Appendix A we present shape models oriented at zero epoch using the ISAM service ${ }^{10}$. On the web page these plots can be set in motion, together with the rotating shape model.

\section{1. (159) Aemilia}

Lightcurves of (159) Aemilia have been previously obtained by Harris \& Young (1989), Behrend et al. (2014), Ditteon \& Hawkins (2007), and Pilcher (2013). Initially there was controversy over whether the rotation period is close to 16 or $24 \mathrm{~h}$; this issue was resolved by Pilcher (2013) based on multiple coverage from the year 2012 folded with a period of $24.476 \mathrm{~h}$. The lightcurve amplitudes varied from 0.17 to $0.26 \mathrm{mag}$.

We observed Aemilia in two other apparitions, in 2014 and 2015. Additionally, we present here unpublished lightcurves from 2005 obtained by the group led by Raoul Behrend and based on incomplete coverage. The morphology of the new lightcurves was similar to previously observed ones; there were characteristic "shelves" after the maxima, one of which had a tendency to evolve to a third maximum when observed at a larger phase angle (Figs. A.7-A.9). The synodic periods of the composite lightcurves were around $24.49 \mathrm{~h}$, with amplitudes from $0.24 \mathrm{mag}$ to $0.18 \mathrm{mag}$.

The dataset for the lightcurve inversions consisted of 45 individual lightcurve fragments from six apparitions (1981, 2005, 2006, 2012-2013, 2014, and 2015), well spread over the asteroid orbit and a range of phase angles (see Table A.1). We did not use the short and noisy fragment from 2008; all the other available data were used in the modelling process. The dataset consisted of around $200 \mathrm{~h}$ of dense lightcurve observations.

In the convex inversion, the spherical harmonics expansion and convexity regularisation weight had to be increased in order to produce realistic physical shape models (Fig. 3). The sidereal period value and both solutions for the spin axis (Table 3) clearly stood out in the parameter space in terms of lowest RMSD (0.014 mag). The example fit to the lightcurves is shown in Fig. 13. The last lightcurve from the apparition in 2014, and the first one from 2015, both obtained at large phase angles, had the worst fit to the model lightcurves. All the resulting shape

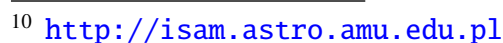

Table 6. Reduced $\chi^{2}$ minimum values of various models fit to infrared data in thermophysical modelling.

\begin{tabular}{|c|c|c|}
\hline Shape model $(\lambda, \beta)$ & Low roughness & High roughness \\
\hline \multicolumn{3}{|c|}{ (159) Aemilia } \\
\hline sphere $\left(139^{\circ},+68^{\circ}\right)$ & 1.16 & 1.21 \\
\hline sphere $\left(348^{\circ},+59^{\circ}\right)$ & 1.15 & 1.21 \\
\hline convex $\left(139^{\circ},+68^{\circ}\right)$ & 0.61 & 0.53 \\
\hline convex $\left(348^{\circ},+59^{\circ}\right)$ & 0.44 & 0.56 \\
\hline $\operatorname{SAGE}\left(139^{\circ},+66^{\circ}\right)$ & 0.44 & 0.47 \\
\hline $\operatorname{SAGE}\left(349^{\circ},+63^{\circ}\right)$ & 0.53 & 0.52 \\
\hline \multicolumn{3}{|c|}{ (227) Philosophia } \\
\hline sphere $\left(95^{\circ},+19^{\circ}\right)$ & 2.67 & 1.34 \\
\hline sphere $\left(272^{\circ},-1^{\circ}\right)$ & 2.67 & 1.45 \\
\hline convex $\left(95^{\circ},+19^{\circ}\right)$ & 2.37 & 1.22 \\
\hline convex $\left(272^{\circ},-1^{\circ}\right)$ & 2.49 & 1.34 \\
\hline $\operatorname{SAGE}\left(97^{\circ},+16^{\circ}\right)$ & 1.93 & 1.28 \\
\hline $\operatorname{SAGE}\left(271^{\circ}, 0^{\circ}\right)$ & 1.93 & 1.40 \\
\hline \multicolumn{3}{|c|}{ (329) Svea } \\
\hline sphere $\left(33^{\circ},+51^{\circ}\right)$ & 1.63 & 1.61 \\
\hline sphere $\left(157^{\circ},+47^{\circ}\right)$ & 1.67 & 1.60 \\
\hline convex $\left(33^{\circ},+51^{\circ}\right)$ & 0.98 & 0.97 \\
\hline convex $\left(157^{\circ},+47^{\circ}\right)$ & 1.38 & 1.17 \\
\hline $\operatorname{SAGE}\left(21^{\circ},+47^{\circ}\right)$ & 1.21 & 1.09 \\
\hline $\operatorname{SAGE}\left(166^{\circ},+39^{\circ}\right)$ & 1.39 & 1.55 \\
\hline \multicolumn{3}{|c|}{ (478) Tergeste } \\
\hline sphere $\left(2^{\circ},-42^{\circ}\right)$ & 2.86 & 2.24 \\
\hline sphere $\left(216^{\circ},-56^{\circ}\right)$ & 2.41 & 1.98 \\
\hline convex $\left(2^{\circ},-42^{\circ}\right)$ & 2.18 & 2.59 \\
\hline convex $\left(216^{\circ},-56^{\circ}\right)$ & 1.53 & 1.81 \\
\hline $\operatorname{SAGE}\left(4^{\circ},-43^{\circ}\right)$ & 1.44 & 1.68 \\
\hline $\operatorname{SAGE}\left(218^{\circ},-56^{\circ}\right)$ & 1.03 & 1.08 \\
\hline \multicolumn{3}{|c|}{ (487) Venetia } \\
\hline sphere $\left(78^{\circ},+3^{\circ}\right)$ & 2.39 & 1.62 \\
\hline sphere $\left(252^{\circ},+3^{\circ}\right)$ & 1.38 & 1.09 \\
\hline convex $\left(78^{\circ},+3^{\circ}\right)$ & 2.01 & 2.69 \\
\hline convex $\left(252^{\circ},+3^{\circ}\right)$ & 1.82 & 2.88 \\
\hline $\operatorname{SAGE}\left(70^{\circ},+8^{\circ}\right)$ & 1.30 & 1.79 \\
\hline $\operatorname{SAGE}\left(255^{\circ},+8^{\circ}\right)$ & 1.04 & 1.23 \\
\hline
\end{tabular}

Notes. The first column gives the shape model type and spin axis position.

solutions were roughly similar to each other. Some shapes resembled a deltoid, while others were more ellipsoidal; there were small differences in the vertical dimensions. Here we present only one of the possible shapes for pole 1, which has been the standard practice in presenting lightcurve inversion solutions.

The non-convex model obtained with the SAGE algorithm fits the lightcurves similarly well (RMSD $=0.014$ mag, Fig. 13) and similar spin solutions were found (Table 3), but the shape is more compact, with slight indentations and some large bulges (Fig. 4). The genetic evolution runs all led to the final shapes that were very similar to each other, and the only differences were in the depth of the largest "basins", which were still present on each final shape. The final solution had spin axis parameters close to the average of all the obtained solutions and had the lowest RMSD.

The fitting to all four solutions (two mirror poles from the convex inversion and two from the SAGE algorithm) to the fourchord occultation from 2 May 2009 (Dunham et al. 2016) does not provide a preferred solution for the pole or shape, but allows us to scale the model (see Fig. 14). The size of both convex 
Table 7. Asteroid diameters from AKARI, IRAS, and WISE compared to values obtained here on combined data for the preferred pole solution (Col. 5) using TPM.

\begin{tabular}{ccccccc}
\hline \hline & & & \multicolumn{3}{c}{ Radiometric solution for combined data } \\
Target & $\begin{array}{c}D_{\text {AKARI }} \\
{[\mathrm{km}]}\end{array}$ & $\begin{array}{c}D_{\text {IRAS }} \\
{[\mathrm{km}]}\end{array}$ & $\begin{array}{c}D_{\text {WISE }} \\
{[\mathrm{km}]}\end{array}$ & $\begin{array}{c}\text { Diameter } \\
{[\mathrm{km}]}\end{array}$ & Albedo & $\begin{array}{c}\text { Thermal inertia } \\
{\left[\mathrm{Jm}^{-2} \mathrm{~s}^{-0.5} \mathrm{~K}^{-1}\right]}\end{array}$ \\
\hline 159 Aemilia & 130.0 & 125.0 & 127.4 & $137 \pm 8$ & $0.054 \pm 0.015$ & $50 \pm 50$ \\
227 Philosophia & 95.6 & 87.3 & 105.3 & $101 \pm 5$ & $0.041 \pm 0.005$ & $125 \pm 90$ \\
329 Svea & 70.4 & 77.8 & 69.2 & $78 \pm 4$ & $0.055 \pm 0.015$ & $75 \pm 50$ \\
478 Tergeste & 85.6 & 79.5 & 77.2 & $87 \pm 6$ & $0.15 \pm 0.02$ & $75 \pm 45$ \\
487 Venetia & 66.1 & 63.1 & 65.6 & $70 \pm 4$ & $0.21 \pm 0.02$ & $100 \pm 75$ \\
\hline
\end{tabular}

Notes. The last two columns contain the derived albedo and thermal inertia values. Errors are full $3 \sigma$ range.

and non-convex models fitted to this occultation yields equivalent volume sphere diameters from 130 to $138 \mathrm{~km}$; the SAGE solutions are a few kilometres larger than the convex models (see Table 4). In Table 7, we present the radiometric size for the model solution that best fits in thermophysical modelling, i.e. $137 \mathrm{~km}$, in very good agreement with the size from occultations.

The application of inversion models of (159) Aemilia in thermophysical modelling is a rare example of a remarkably good fit with no trend in the $\mathrm{O}-\mathrm{C}$ plots (see Figs. 15). These O-C plots show nicely if a given model solution (size, shape, thermal properties) can explain all the thermal measurements simultaneously. Ratios close to 1.0 (solid line) indicate an excellent match between observation and the corresponding model prediction; ratios in the range 0.9 and 1.1 (dashed lines) reflect typical calibration uncertainties of thermal measurements. As a rule of thumb, a $10 \%$ flux error roughly translates into a $5 \%$ error in the object's radiometric size solution. Finding many data points outside the $+/-10 \%$ lines usually indicates that the shape/spin solution has some problems. Therefore, systematic offsets in the $\mathrm{O}-\mathrm{C}$ plots indicate a problem with the radiometric size solution. Strong trends in the Obs/TPM ratio with wavelength point towards problems with the thermal surface properties (thermal inertia and roughness), an asymmetry in the preand post-opposition ratios are connected to an incorrect thermal inertia, while outliers in the rotational-phase plot point to shaperelated issues. We used $H=8.100 \mathrm{mag}$ and $G=0.09$, after Pravec et al. (2012), and infrared data from IRAS $(6 \times 4$ band detections), AKARI (5 datapoints), and WISE W3/W4 bands (20 datapoints). Both convex and non-convex models with both pole solutions fit the data similarly well, and substantially better than a spherical model (see Table 6).

The first model solution from the SAGE method $\left(\lambda=139^{\circ}\right.$, $\beta=66^{\circ}$ ) seems to be the overall best solution (the reduced $\chi^{2}$ of 0.44 ) and intermediate level of surface roughness, optimum thermal inertia around 50 SI units (higher for higher roughness, lower for lower roughness), effective size of around $137.0 \mathrm{~km}$ (around $10 \mathrm{~km}$ larger than in previous determinations), and geometric $V$-band albedo of 0.054 . Uncertainty values can be found in Table 7. The radiometric size is in agreement with lower values for the size from occultation fitting, but is still slightly higher than in all previous determinations that used a spherical model for the shape, also partly due to lower albedo than in previous works (see Table 7).

\section{2. (227) Philosophia}

(227) Philosophia has been observed by many authors, e.g. Bembrick et al. (2006), Ditteon \& Hawkins (2007), Behrend et al. (2014), Alkema (2013), Pilcher \& Alkema (2014a,b), but the controversy regarding its rotation period remains (see our discussion on this target in M2015). In our previous work we considered a period of $26.46 \mathrm{~h}$ as the most probable, based on our monomodal lightcurve from the apparition on the verge of 2013 and 2014. Still, the currently accepted value in LCDB is twice as long, $52.98 \mathrm{~h}$; however, it is annotated as not fully certain and ambiguous (code 2, and label A). The reported amplitudes ranged from 0.06 to $0.20 \mathrm{mag}$, but these values can be influenced by incorrect periods used for folding the lightcurves.

During the observing campaign within this work, we obtained extensive datasets from two more apparitions of Philosophia, in 2015 and 2016, in addition to the one from 2013-2014. In both of them a clearly bimodal behaviour over the shorter period timescale has been recorded, which resolves the problem of uncertain period, confirming our value of $26.46 \mathrm{~h}$ (see Figs. A.11 and A.12). This period fits all the available data from previous apparitions. In additional, we present here the data from apparition in 2006 from Behrend et al. (2014) and Ditteon \& Hawkins (2007) folded together (Fig. A.12). Overall, the behaviour of the lightcurve variations changes from monomodal to bimodal with minima of unequal depth, and other irregularities. Curiously, monomodal lightcurves of this target do not display smaller amplitudes than bimodal ones, contrary to what is usually the case; instead, the amplitude remains on a stable level of around $0.15 \mathrm{mag}$ in all apparitions.

Unfortunately, the data from the years 2004 and 2005 were not available. For the modelling, we used all the other data from five distinct apparitions (2006, 2012-2013, 2013-2014, 2015, and 2016); there are as many as 97 separate lightcurve fragments, covering a total of around $500 \mathrm{~h}$. The modelling with the convex inversion method clearly pointed to two strong solutions for the spin axis, which appeared to have low inclination to the ecliptic (Table 3), as was expected from the lightcurve morphology changes. The shape model is quite atypical, with a triangular appearance when viewed from the equator (Fig. 5). This shape actually caused the most problems in the convex inversion as almost all the resulting shapes had an axis of greatest inertia tensor not coincident with the spin axis, regardless of the starting parameters. We present here two solutions where the difference between the rotation axis and the axis of greatest inertia were smallest. The fit to the lightcurves is satisfactory (RMSD $=0.011 \mathrm{mag}$, Fig. 16) with the exception of the first two lightcurves from the year 2015 .

The SAGE algorithm also had problems with modelling this target. Some evolutionary paths were stuck in a blind track and finding a unique solution took much more CPU time than usual (one week compared to two days on the cluster consisting of ten 6-core $3 \mathrm{GHz}$ AMD processors and $2 \mathrm{~GB}$ RAM). Finally, two sets of solutions for the pole and shape were found (Table 3, 

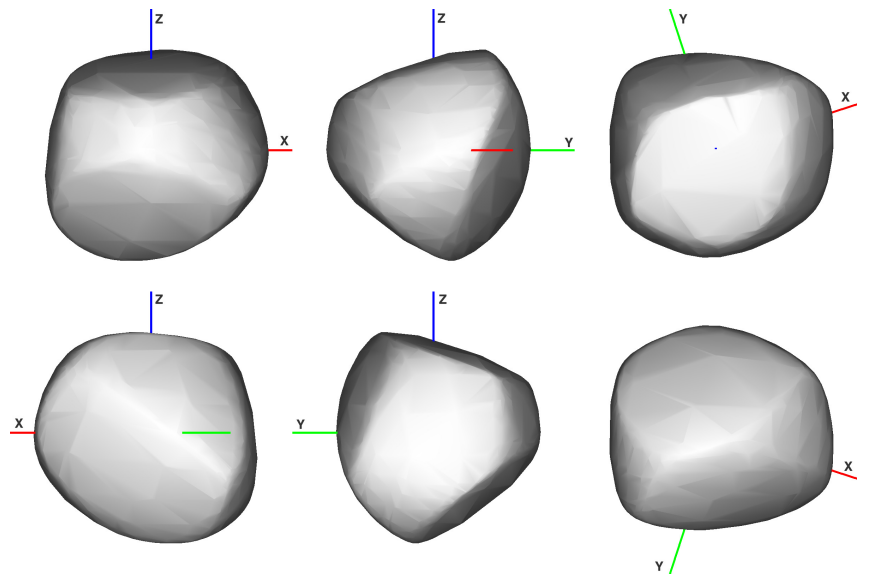

Fig. 5. Convex shape model of (227) Philosophia from the lightcurve inversion method shown in six projections.
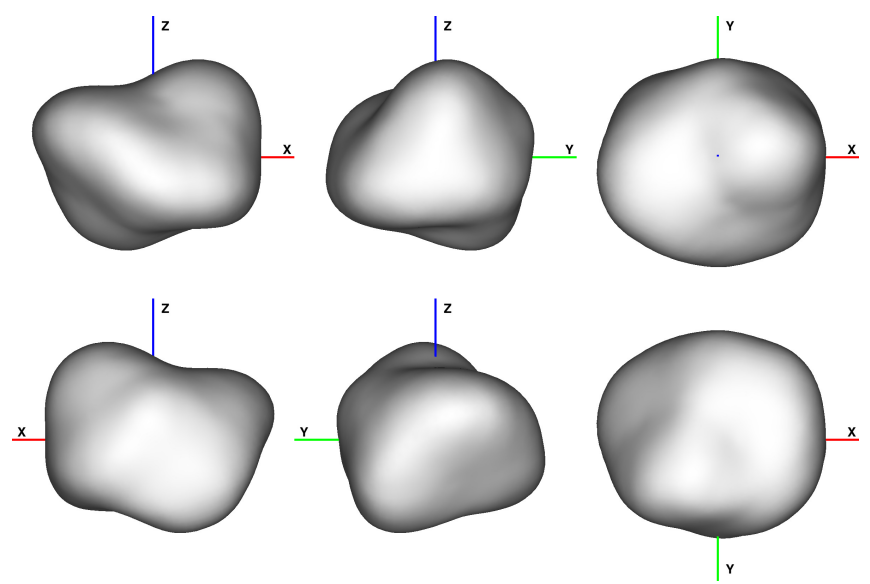

Fig. 6. Non-convex shape model of (227) Philosophia from the SAGE algorithm shown in six projections.

Fig. 6); however, when starting the evolution around the expected mirror solution, the process often ended up near the other pole. Most probably, the mirror solution had the incorrect inertia tensor, thus was often rejected by the algorithm. Still, as the results from the convex inversion suggest, both pole solutions can fit the data on a similar level, so we consider the mirror pole solution equally possible. Here, the two above-mentioned lightcurves also fit worse than all the other fragments, and the overall RMSD value is 0.009 mag. The non-convex shape model of Philosophia is even more specific: one lobe is substantially larger than the other, and there are many strongly non-convex features. However, its pole-on outline largely coincides with the corresponding solution from the convex inversion.

In thermophysical modelling, Philosophia turned out to be the worst constrained case of the five targets studied here. Actually, the convex and SAGE models fit to thermal data was only slightly better than the corresponding spherical shape solution with the same spin parameters, indicating that inversion shape solutions are not yet perfect. We used an $H$ value equal to $9.1 \mathrm{mag}$ and a $G$ value equal to $0.15^{11}$, and thermal data from IRAS (16 measurements), AKARI (6), and WISE W3/W4 (17). It seems that high-roughness solutions are favoured (Table 6).

The overall best fit in TPM is found for the first convex solution $\left(\lambda=95^{\circ}, \beta=+19^{\circ}\right)$ with a $\chi^{2}$ of 1.2 . The model fits best for a high level of surface roughness, optimum thermal inertia around 100-150 SI units, effective size in the range of

\footnotetext{
${ }^{11}$ After: https://mp3c.oca.eu
}
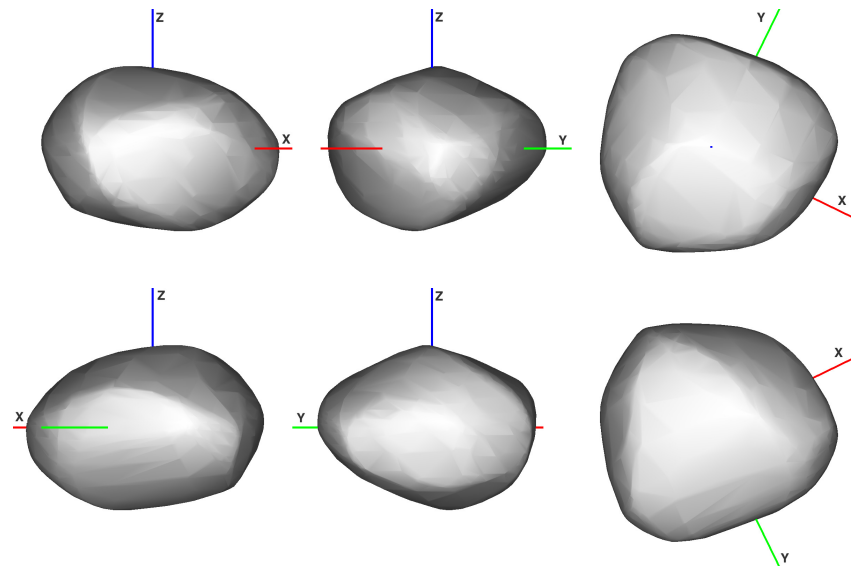

Fig. 7. Convex shape model of (329) Svea from the lightcurve inversion method shown in six projections.
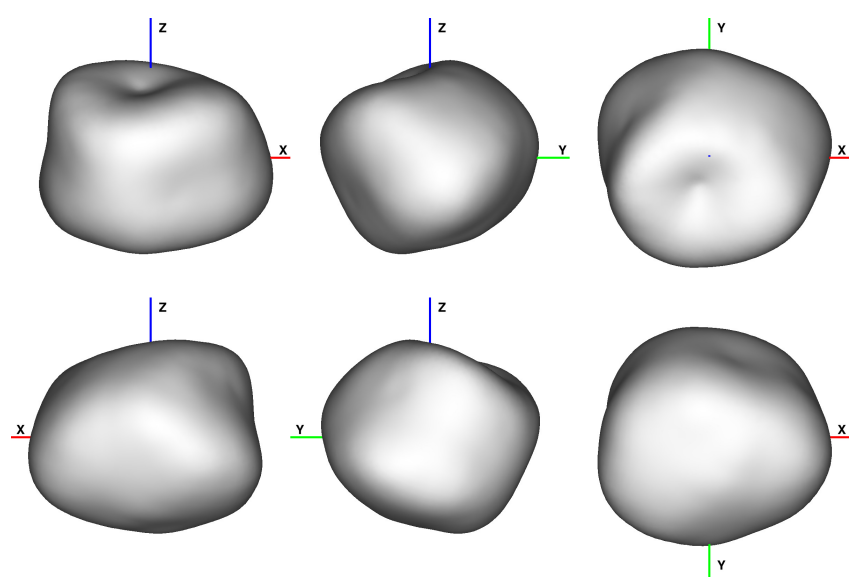

Fig. 8. Non-convex shape model of (329) Svea from the SAGE algorithm shown in six projections.

91-105 km (in agreement with previous determinations), and geometric $V$-band albedo of $0.038-0.044$ (Table 7).

One explanation for this behaviour of the models is that the data are not well balanced with respect to phase angles: there is only one data point at a negative phase angle (i.e. before the opposition). There is no clear trend with wavelength or with rotational phase (Fig. 17), but the data quality is not optimal. Also, the low pole of Philosophia might be the source of the problems; in pole-on geometries for many months one of the hemispheres is heated constantly and that heat can penetrate to much deeper layers which have different thermal properties from the surface regolith. For a change, in geometries closer to equator-on, there are normal diurnal variations in the heat wave. Unfortunately, there is no multi-chord stellar occultation by Philosophia for comparison with the radiometric parameters or topographic features of the models obtained here.

\section{3. (329) Svea}

Svea is one of the first targets from our survey for which we found substantially different period than that accepted in LCDB (see M2015). Observed previously by Weidenschilling et al. (1990), Pray (2006), Menke et al. (2008), and Behrend et al. (2014), Svea displayed the ambiguous periods $15.201 \mathrm{~h}$ or $22.778 \mathrm{~h}$. In M2015, we confirmed a $22.78 \mathrm{~h}$ period based on data from the year 2013, and since that time we have gathered data from two more apparitions, in 2014, and 2016. The lightcurve morphology of Svea is interesting and strongly variable; from clearly trimodal, through almost flat, to the more 

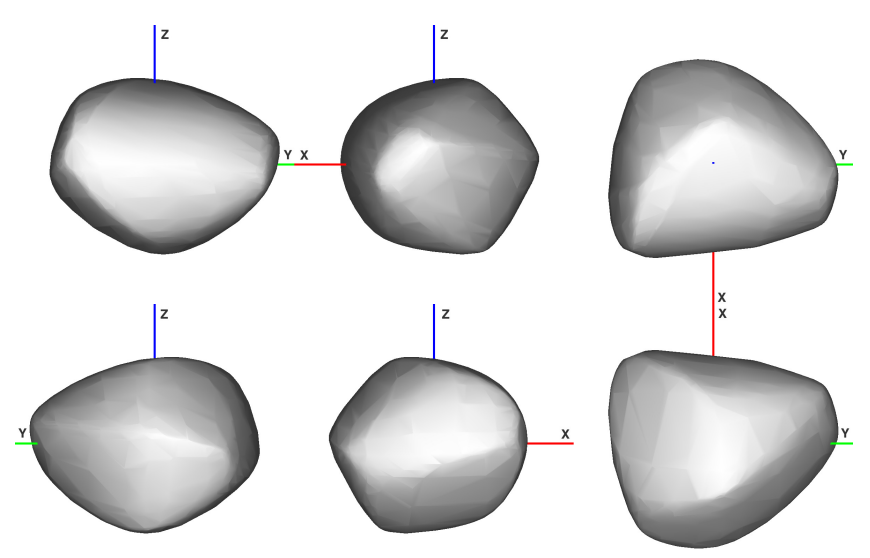

Fig. 9. Convex shape model of (478) Tergeste from the lightcurve inversion method shown in six projections.
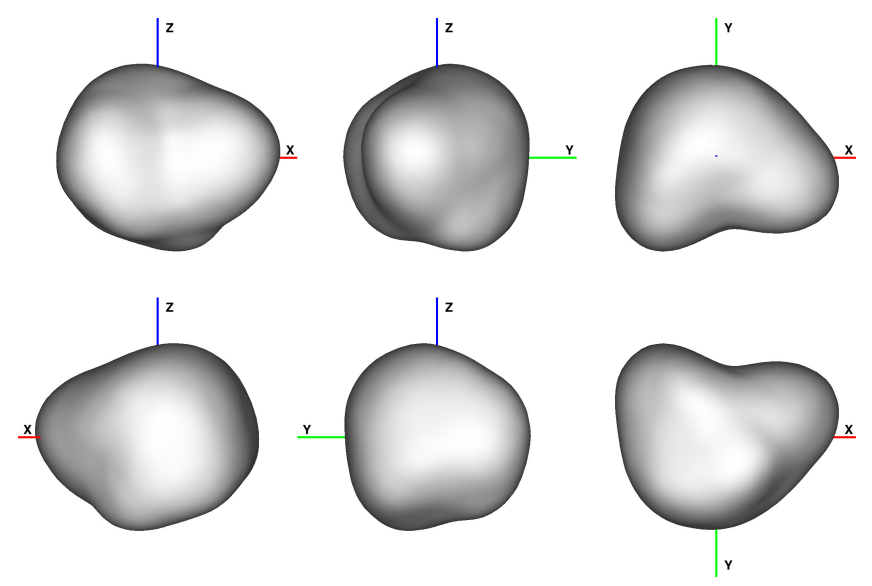

Fig. 10. Non-convex shape model of (478) Tergeste from the SAGE algorithm shown in six projections.

usual bimodal lightcurve of larger amplitude (see Figs. A.14 and A.15). Available data from all apparitions fit the $22.78 \mathrm{~h}$ period, and display amplitudes from 0.09 to $0.24 \mathrm{mag}$.

For the modelling, we were able to use our data from three apparitions coupled with data from 2005 provided by Menke et al. (2008), from 2006 by Behrend et al. (2014), and only one of the four lightcurves from 1986 saved as a composit by Weidenschillig (1990). In total, there are 60 lightcurve fragments from six apparitions.

In the modelling by the convex lightcurve inversion method, two resulting pole solutions were closer together than in the usual miror-pole symmetry, differing by only $124^{\circ}$ in ecliptic longitude, with a similar values for pole latitude (Table 3 ). The shape model vertical dimensions were not well constrained, but the other features were stable (Fig. 7), providing a good fit to lightcurves at $0.010 \mathrm{mag}$ level for both pole solutions (Fig. 18).

The SAGE spin solutions were $145^{\circ}$ apart (Table 3 ) and the corresponding shape models showed some large indentations near the equator and one of the poles (Fig. 8). The fit to the lightcurves shows $0.011 \mathrm{RMSD}$ and is very similar to the fit by the convex models (Fig. 18).

In the case of Svea, there are two very good multi-chord occultations available (Dunham et al. 2016) observed from Japan in 2011 (7 chords), and from Florida, USA, in 2013 (6 chords), giving a rare opportunity to test the shape models down to the medium-scale details. Additionally, in these events, a few negative results were recorded, allowing for better size constraints. Appendix B lists occultation observers and site names.

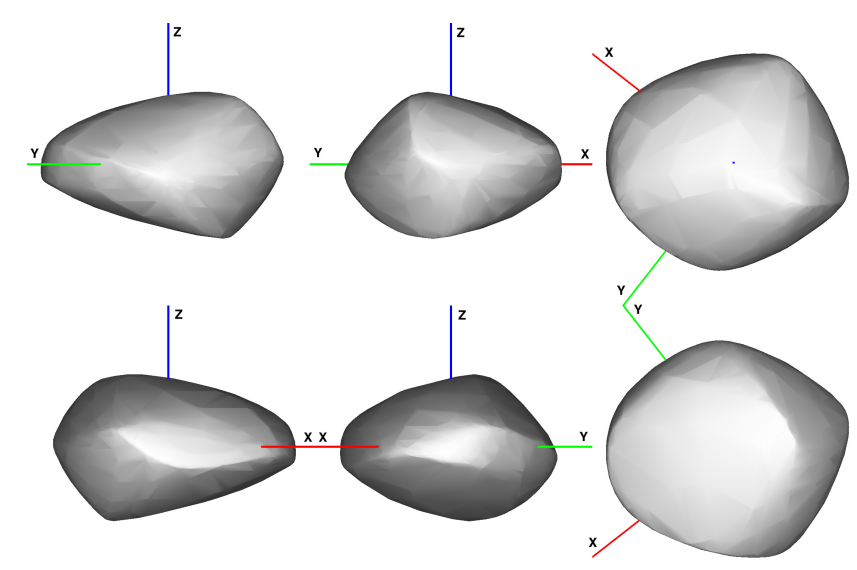

Fig. 11. Convex shape model of (487) Venetia from the lightcurve inversion method shown in six projections.
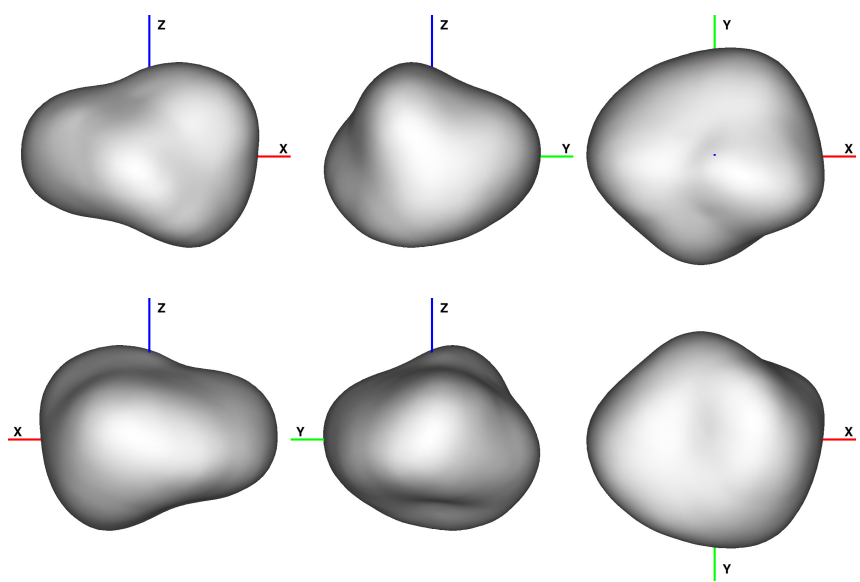

Fig. 12. Non-convex shape model of (487) Venetia from the SAGE algorithm shown in six projections.

Fitting our models of Svea to these occultations gave remarkably good results, clearly allowing us to reject one of the mirror pole solutions (pole 2, shown in Fig. 20), and confirming the first pole solution with indentations and other shape features of the SAGE model (Fig. 19). The convex model for pole 1 also fits both occultations well, but the non-convex model fits markedly better. This way the model gets unique validation and it shows that major topographic features present in the non-convex models made with SAGE are confirmed when auxiliary data are available. The fitting to two occultation events was done independently, but the results are internally consistent. Obtained size estimates range from 70 to $74 \mathrm{~km}$ for the effective diameter (see Table 5), which agrees with the radiometric size $(77.5 \mathrm{~km}$ in Table 7) within the error bars.

Curiously, in thermophysical modelling it is the convex model (but also pole 1) that is slightly preferred. However, all the inversion solutions clearly fit better to the thermal data than does the corresponding spherical shape solution with the same spin properties. In TPM the preference of pole 1 over pole 2 is stronger than the preference of the best fitting convex model over the non-convex solution; however, all the fits are at an acceptable level (see Table 6). Overall, the thermal data seem to point towards a spin axis close to $\lambda=33^{\circ}$ and $\beta=+51^{\circ}$. The convex inversion solution for this pole provides an excellent fit to all thermal data (reduced $\chi^{2}$ below 1.0) with an intermediate level of surface roughness, optimum thermal inertia around 75 SI units, 
A. Marciniak et al.: Long-period and low-amplitude asteroids
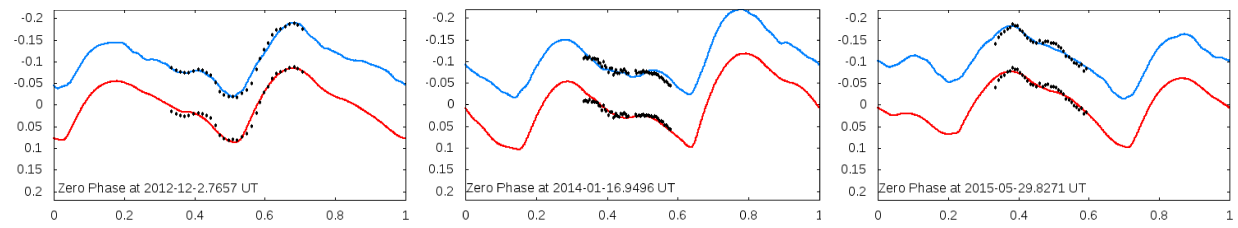

Fig. 13. Convex (upper curve) and non-convex (lower curve) model lightcurves of (159) Aemilia fitted to data from various apparitions (black points).

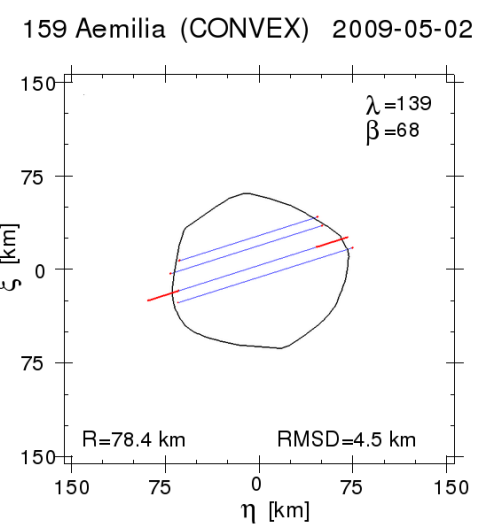

159 Aemilia (SAGE) 2009-05-02

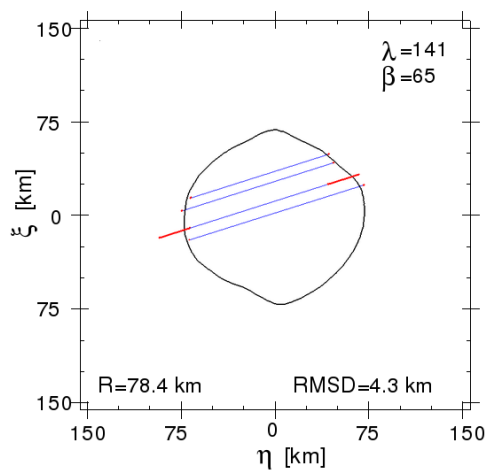

159 Aemilia (CONVEX) 2009-05-02

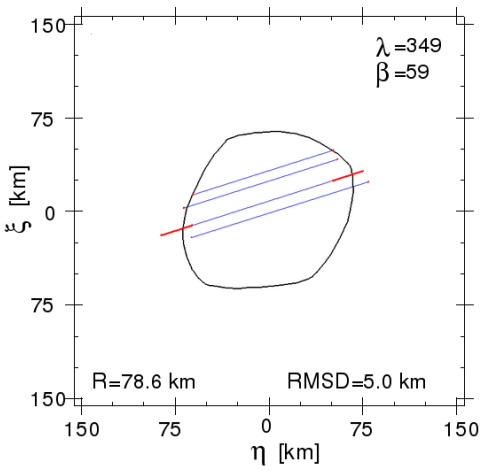

159 Aemilia (SAGE) 2009-05-02

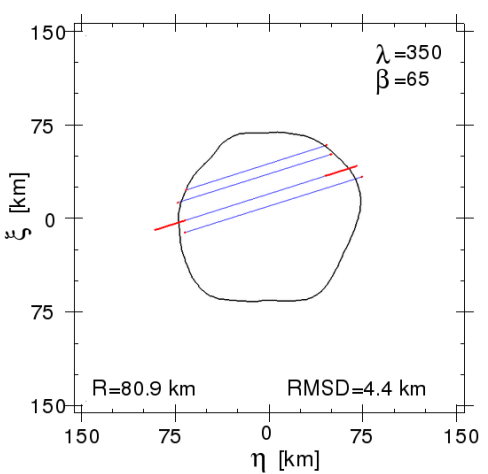

Fig. 14. Stellar occultation fits of convex (top) and non-convex (bottom) models of (159) Aemilia. At the end of each chord a timing uncertainty is marked. $R$ is the radius of the largest model dimension. For equivalent volume sphere diameters see Table 4. effective size of around $77.5 \mathrm{~km}$ (confirming the value from occultations), and geometric $V$-band albedo of 0.055 . The $\mathrm{O}-\mathrm{C}$ plots for the best solution are shown in Fig. 21, and the uncertainties on the derived values are given in Table 7 . The infrared data that were used came from IRAS (20 measurements), AKARI (9), Wise W3/W4 (28), and MSX (8), and the adopted absolute magnitude and slope were 9.34 and 0.04 , respectively.

\section{4. (478) Tergeste}

Asteroid (478) Tergeste was observed previously for lightcurves in only two apparitions, by Harris \& Young (1989) and Behrend et al. (2014). In the latter, it displayed a $0.22 \mathrm{mag}$ amplitude lightcurve of $16.104 \mathrm{~h}$ period. We observed it in our project since 2013 through four consecutive apparitions, confirming the period around $16.104 \mathrm{~h}$ and registering lightcurves of 0.15 up to 0.30 mag amplitudes. Those with larger amplitudes showed sharp minima and wide asymmetric maxima, while others were smoother and more regular (see Figs. A.16 to A.20).

For the modelling, we used a dataset consisting of 48 lightcurves from six apparitions (in 1980, 2005, 2013, 2014, 2015, and 2016). In the convex inversion, a convexity regularisation weight had to be slightly increased in order to make some shape models physical (rotating around the axis of greatest inertia tensor). There are two narrow solutions for the pole in the parameter space (Table 3), and the shape models are trapezoidal (Fig. 9). The fit to the lightcurves is on a $0.011 \mathrm{mag}$ level (see Fig. 22).

The non-convex SAGE models confirm these pole solutions within the small error bars (Table 3), but here the shapes are more complex, e.g. with a large valley visible from the pole-on view (Fig. 10) in a place where the convex models showed a straight, planar area. Both spin solution models provide a similar fit to lightcurves (0.011 mag) Fig. 22. However, the Tergeste model fit (see Sect. 3.1) shows the tendency of non-convex models to better fit deep and sharp lightcurve minima (see middle plot of Fig. 22). These local features, with only a few datapoints, cannot notably influence the overall RMSD value, but they clearly need some shadowing to be correctly reproduced (see the shape model projection in Fig. A.17).

There is no multi-chord stellar occultation to discriminate between two equally possible pole solutions from lightcurve inversion for Tergeste, but surprisingly the thermophysical modelling shows a strong preference for one of the spin and shape solutions (Table 6). non-convex model 2 (at $\lambda=218^{\circ}, \beta=$ $\left.-56^{\circ}\right)$ provides a very good fit to the thermal data $\left(\chi^{2}\right.$ around 1.0), while the other inversion spin and shape solutions give fits that are at least 1.5 times worse (at the edge of being acceptable), and the spherical model gives a fit that is 2.5 times worse. The preferred solution provides a very good fit to 

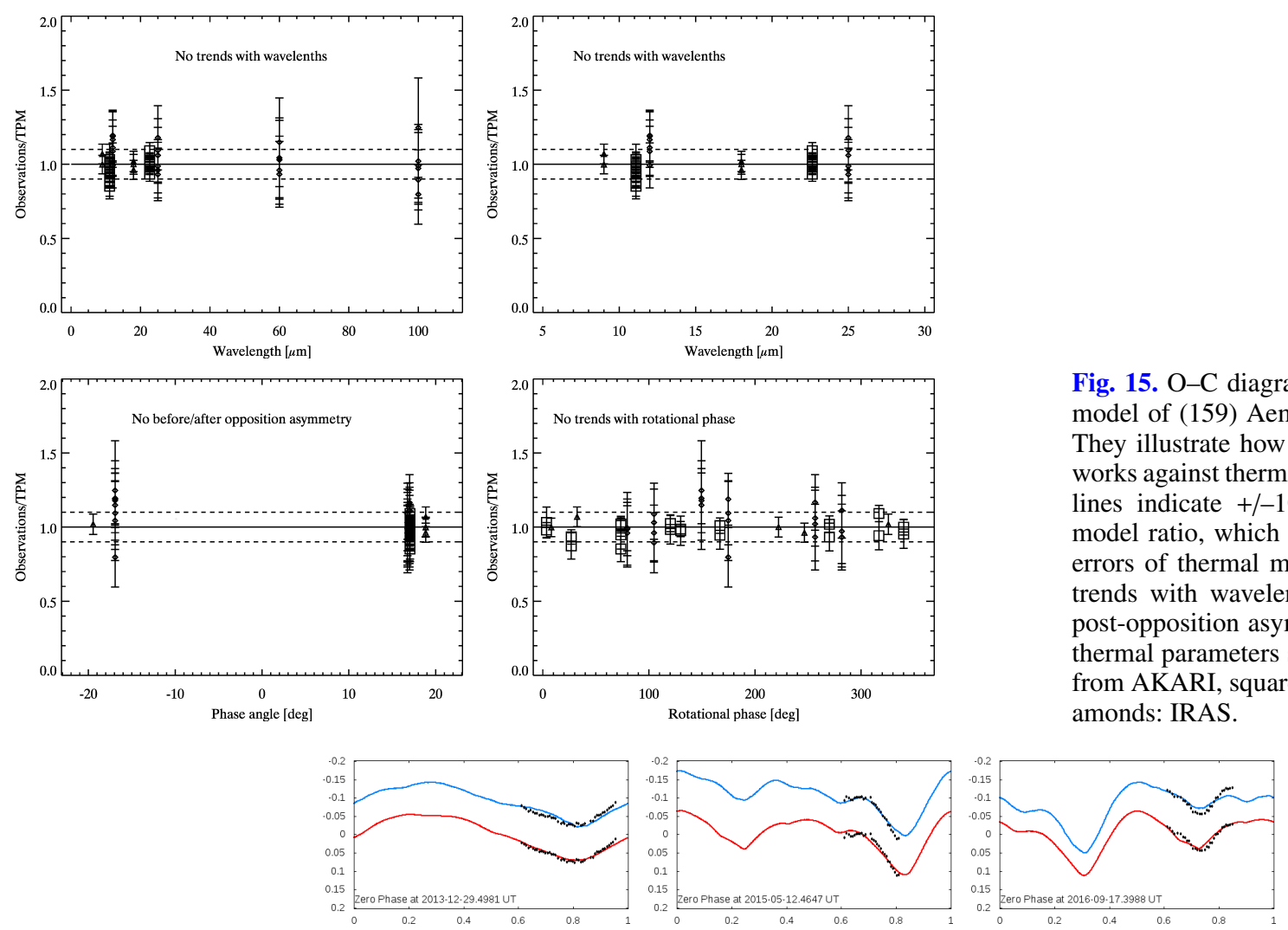

Fig. 16. Convex (upper curve) and non-convex (lower curve) model lightcurves of (227) Philosophia fitted to data from various apparitions (black points). and 18 from WISE $W 3 / W 4$ bands, adopting $H=7.96$ and $G=0.15$; Fig. 23) with an intermediate level of surface roughness, optimum thermal inertia around 75 SI units, effective size around $87.3 \mathrm{~km}$, and geometric $V$-band albedo of 0.15 (the last two values are closest to AKARI determinations, see Table 7).

\section{5. (487) Venetia}

Observed previously in as many as six apparitions, (487) Venetia displayed lightcurves of varying shape and amplitude. However, some of the observations only partially covered its $13.34 \mathrm{~h}$ lightcurve (Weidenschilling et al. 1990; Shevchenko et al. 1992; Neely 1992; Schober et al. 1994; Ferrero 2014; Behrend et al. 2014).

Erikson et al. (2000) and Tungalag et al. (2002) published spin and shape solution for Venetia with similar spin axis coordinates, but a notable difference in sidereal period:

Erikson et al. (2000) $\lambda_{p}=268^{\circ}, \beta_{p}=-24^{\circ}, P=13.34153 \mathrm{~h}$ Tungalag et al. (2002) $\lambda_{p}=259^{\circ}, \beta_{p}=-30^{\circ}, P=13.33170 \mathrm{~h}$.

We observed Venetia over three consecutive apparitions, registering full lightcurves that were often almost featureless, while in other apparitions it showed a substantial amplitude of 0.23 mag (Figs. A.22-A.24). This behaviour is a strong indication of an elongated object with low inclination of the spin axis.

The lightcurve inversion indeed resulted in very small pole latitudes (see Table 3 ). The convex inversion model displays a somewhat angular flattened shape (Fig. 11), while the SAGE model has a smoother and more complex appearance (Fig. 12). Both model types failed to reproduce tiny but complex the thermal data (28 datapoints from IRAS, 8 from AKARI,
Fig. 15. O-C diagrams for the thermophysical model of (159) Aemilia using SAGE model 1. They illustrate how well the spin/shape model works against thermal infrared data. The dashed lines indicate $+/-10 \%$ in the observation-tomodel ratio, which corresponds to typical flux errors of thermal measurements. There are no trends with wavelength, rotation, or pre- and post-opposition asymmetry. For the best fitting thermal parameters see Table 7. Triangles: data from AKARI, squares: WISE W3/W4, small diamonds: IRAS. brightness variations from pole-on geometries (Fig. 24) at the level of a few $0.01 \mathrm{mag}$, revealing the limits of lightcurve inversion. However, generally the fit was very good at the level of 0.011 mag RMSD in both methods. Our models are close in sidereal period to the value determined by Erikson et al. (2000), and in pole longitude to both pole solutions published by Erikson et al. (2000) and Tungalag et al. (2002); however, they disagree in pole latitude. Our slightly positive values are far from both of the previous determinations (Table 3 ).

Here too there are no available stellar occultations to verify or confirm one of the spin and shape solutions. However, thermophysical modelling shows a similarly strong preference for one of the spin and shape solutions, as in the previous case of (478) Tergeste. Best $\chi^{2}=1.04$ is as much as two times better for the (487) Venetia non-convex model at $\lambda=255^{\circ}, \beta=+8^{\circ}$ than for any of its convex models, and $25 \%$ better than its mirror nonconvex counterpart (see Table 6). The thermal data came from IRAS (32 measurements), AKARI (7), and WISE W3/W4 (46), with adopted $H$ and $G$ values of 8.14 , and 0.15 , respectively. The best fitting thermophysical parameters are an intermediate level of surface roughness, optimum thermal inertia of around 100 SI units, effective size $\sim 69.5 \mathrm{~km}$, and geometric $V$-band albedo of 0.21 (see Table 7). The thermal data used here were well-balanced with pre- and post-opposition geometries, also in the WISE data. One small issue is the small sinusoidal trend with rotational phase visible in the WISE data (squares in Fig. 25). It might indicate some imperfections in the shape model or alternatively the increased infrared flux contribution of surface layers underneath the skin depth from pole-on geometries. Some discrepancy can also be found for one WISE dataset compared to the model of (478) Tergeste (Fig. 23), also a possible indicator of some missing shape features. Unfortunately, the WISE $W 1$ data 

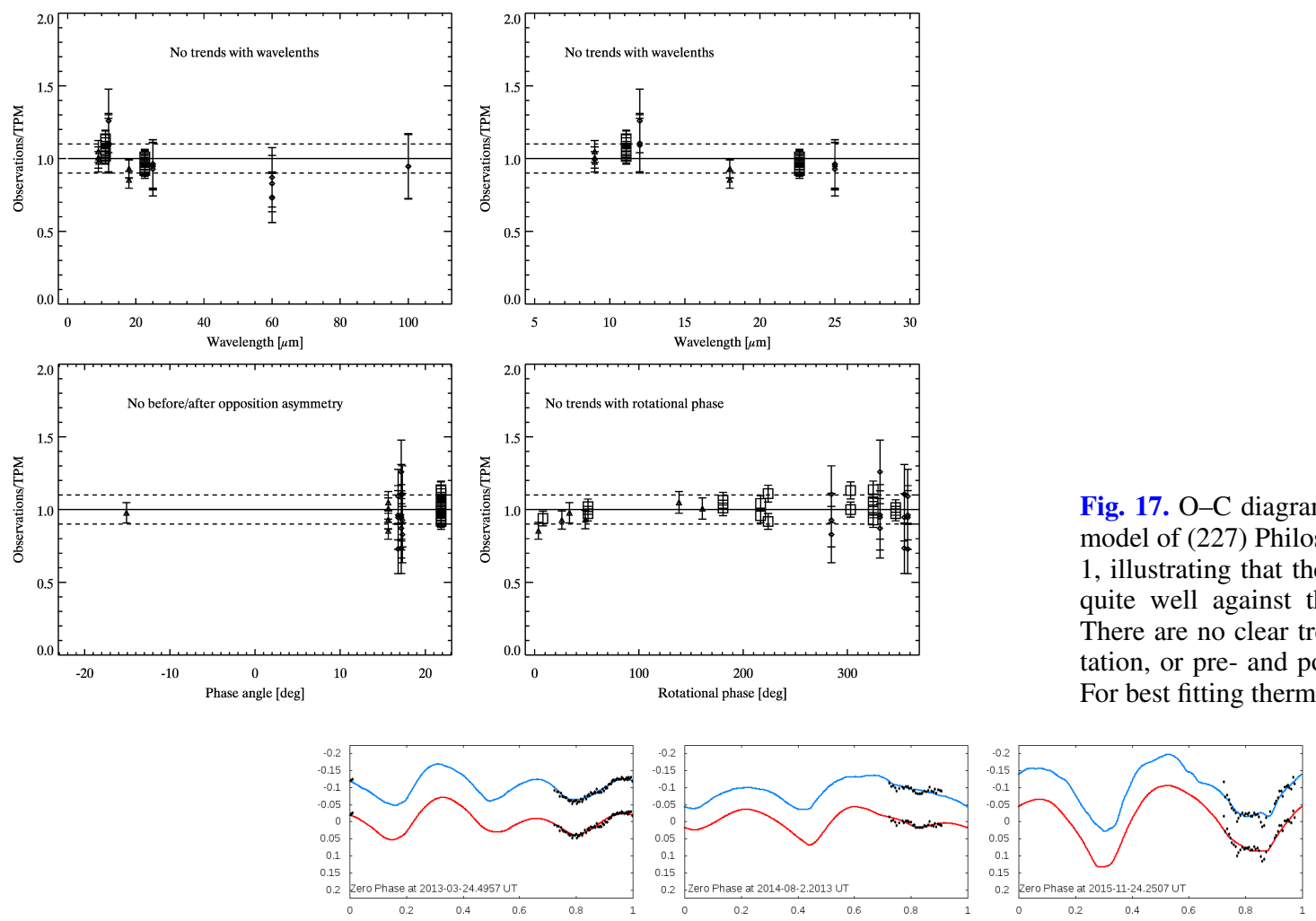

Fig. 18. Convex (upper curve) and non-convex (lower curve) model lightcurves of (329) Svea fitted to data from various apparitions (black points).

are too few and sparse to change the shape models when used as purely reflected light in parallel with all the lightcurve data.

\section{Summary and future work}

This work is a first step towards actual debiasing the available set of spin and shape models for asteroids to include real targets of abundant group with long rotation periods and low amplitude lightcurves. We determined here spin and scaled shape solutions with albedo and thermal inertia values for the first five asteroids from our sample. The diameters are in most cases in good agreement with previous determinations from the IRAS, AKARI, and WISE surveys, though our values are usually a few kilometres larger. The reason for this small discrepancy might be that the cited sizes are usually based on single-epoch measurements, i.e. corresponding more to the apparent cross-section, and on a simple thermal model. The radiometric results obtained here are based on multiple wavelength, epoch, phase-angle, and rotational-phase data, and refer to the scaling size for a given $3 \mathrm{D}$ shape solution.

Spin and shape models, and thermal inertia values for these targets are determined here for the first time (except for (487) Venetia). When most of our sample is modelled and applied this way, the existing bias in these parameters will be largely diminished, at least for bright targets (i.e. for most of large and medium-sized main belt asteroids). We predict that we will complete the task over the course of the next three years.

Our results based on five test cases have shown that asteroid models obtained with both convex and non-convex lightcurve inversion are largely comparable. In some applications (ocultation fitting and thermophysical modelling); however, non-convex models often do somewhat better, sometimes even allowing a choice between two mirror pole solutions. Thanks to the large amount and the high quality of the data used, both model types are smooth and fit the data close to noise level. The differences between the shape models do not manifest themselves in the RMSD value, but they do in the subtle details of the lightcurve fit.

On the contrary, models based on sparse data are usually characterised by low-resolution angular shapes that tend to be problematic in further applications like the above. Nonetheless, sparse data models are good for general statistical studies of spin properties, provided that the data are properly debiased, which is not a trivial task (see e.g. Cibulková et al. 2016). As the Gaia mission is expected to provide absolute photometric data of much better accuracy than previously used sky surveys, some of the biases described in this work are expected to decrease, like those against long-period targets with large amplitudes. Still, to a large extent, low-amplitude targets are going to be problematic for the Gaia mission algorithm for asteroid modelling, as has been shown by Santana-Ros et al. (2015). A substantial amount of low-amplitude asteroids (even up to $80 \%$ of targets with equivalent ellipsoid dimensions $a / b \leq 1.25$, especially those with poles of low inclination to the ecliptic) will be either rejected or wrongly inverted by this algorithm. Thus, it is essential to focus ground-based photometric studies on these more demanding targets to make the well-studied population as complete and varied as possible, and also to start to alleviate biases expected in the future.

Some of our targets that should soon be modellable coincide with asteroids for which the Gaia mission is expected to provide reliable mass estimates, so after scaling them, e.g. by thermophysical modelling, it will be possible to calculate their densities. Practically all of our targets are characterised by complex lightcurves, i.e. a certain signature of asymmetric, complex shapes. Approximating these shapes with simple ellipsoids (as in the Gaia algorithm for asteroids, Cellino et al. 2009) can 


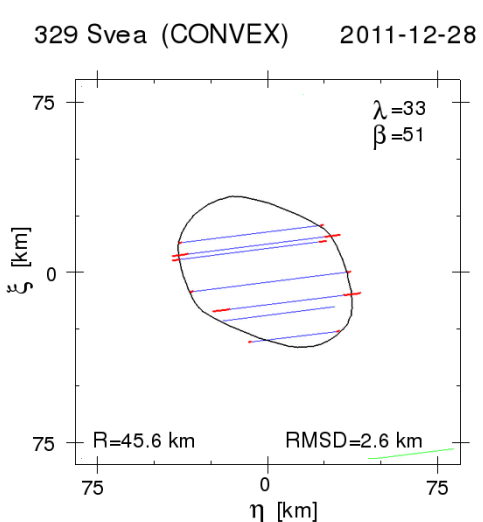

329 Svea (SAGE) 2011-12-28

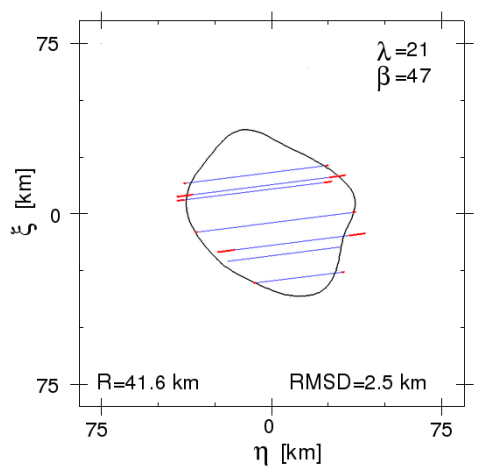

329 Svea (CONVEX) 2011-12-28

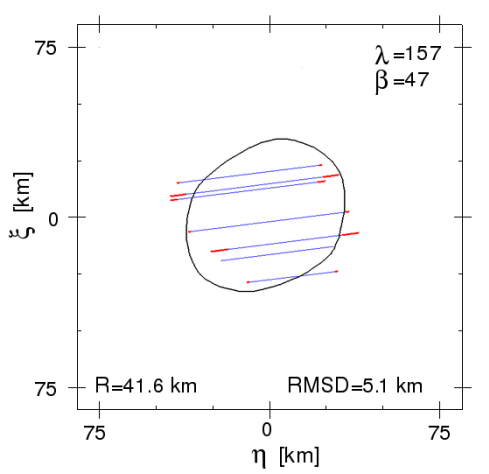

329 Svea (SAGE) 2011-12-28

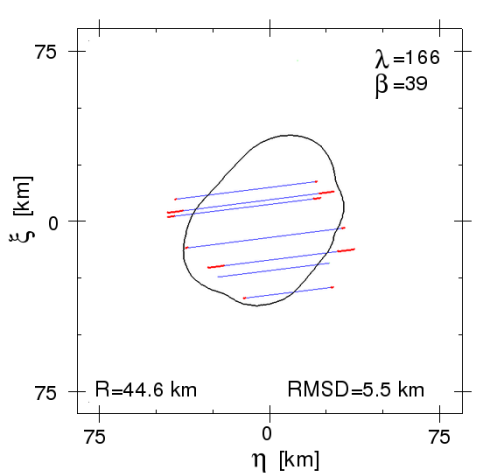

329 Svea (CONVEX) 2013-03-07

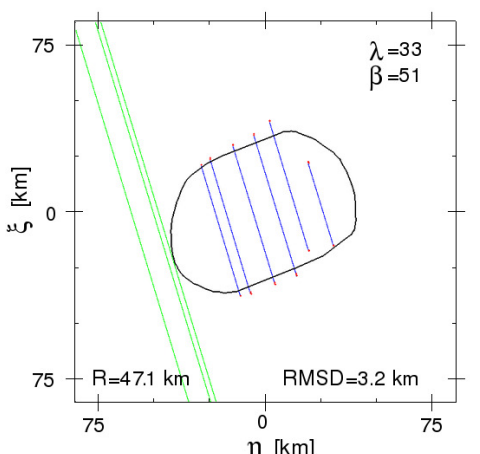

329 Svea (SAGE) 2013-03-07

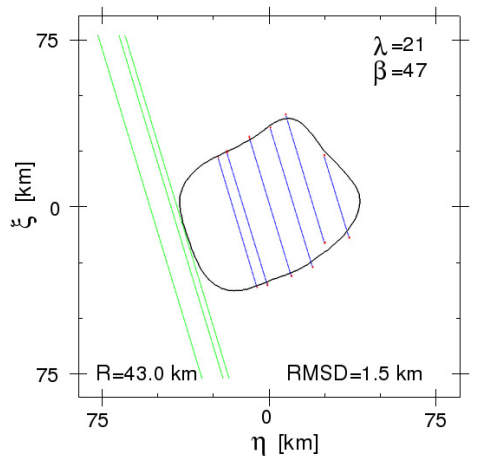

329 Svea (CONVEX) 2013-03-07

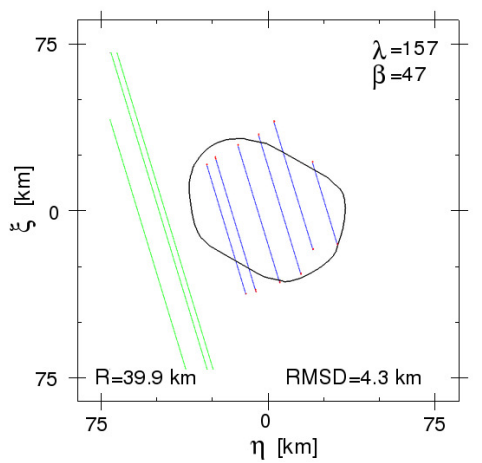

329 Svea (SAGE) 2013-03-07

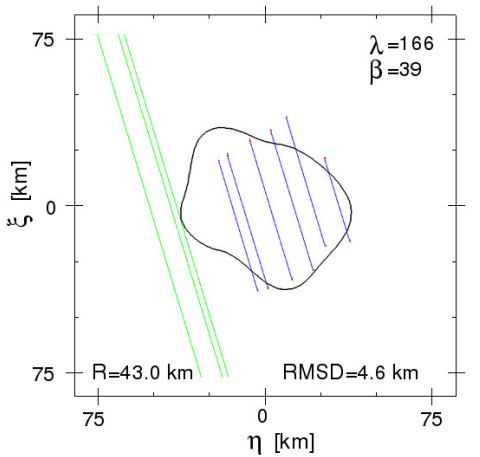

Fig. 19. Two stellar occultation fits of convex (top) and non-convex (bottom) models of (329) Svea, pole 1. At the end of each chord a timing uncertainty is marked. $R$ is the radius of the largest model dimension.
Fig. 20. Svea occultation fits for mirror pole solution (pole 2 from Table 3). The clear misfit of this pole solution allows it to be safely rejected in favour of the pole 1 solution (compare Fig. 19). lead to large errors in derived volumes, which would consequently propagate to large errors in densities (e.g. Carry 2012). Our modelling is going to provide precise shape models that can be further validated and scaled using stellar occultations, adaptive optics imaging, or thermophysical modelling. This way the derived volumes and densities should be possibly closest to real values.
Since most of our targets are bright, both in the visible and the infrared range, many of them have thermal data of good quality, and some even have continuous thermal lightcurves, which - coupled with reliable shape models - are a good input for thermophysical modelling and further studies on their physical parameters (e.g. thermal inertias, albedoes, and sizes) and also on the development of the TPM method itself. 
A. Marciniak et al.: Long-period and low-amplitude asteroids
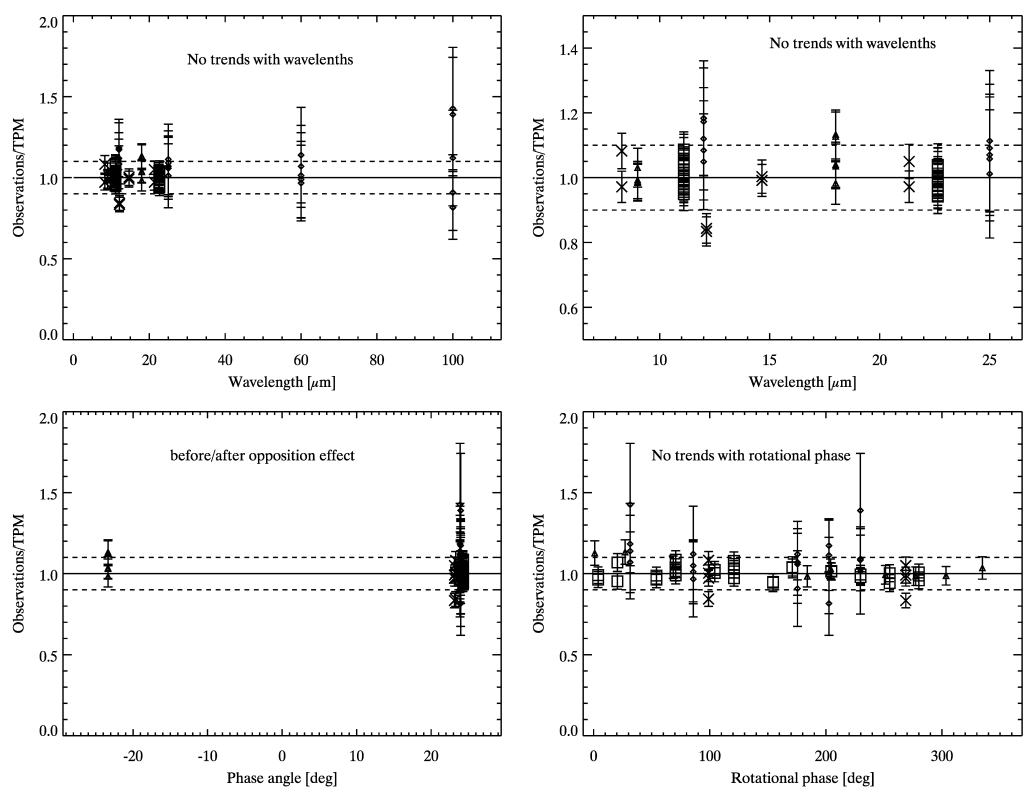

Fig. 21. O-C diagrams for the thermophysical model of (329) Svea using convex model 1. There are no trends with wavelength, rotation, or pre- and post-opposition asymmetry. For the best fitting thermal parameters see Table 7. Triangles: data from AKARI, squares: WISE W3/W4, small diamonds: IRAS, X-symbols: MSX.
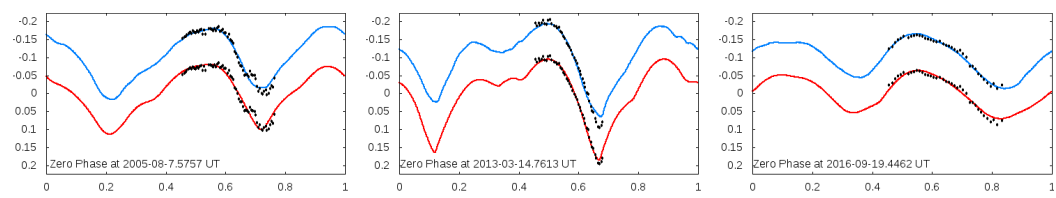

Fig. 22. Convex (upper curve) and non-convex (lower curve) model lightcurves of (478) Tergeste fitted to the data from various apparitions (black points).
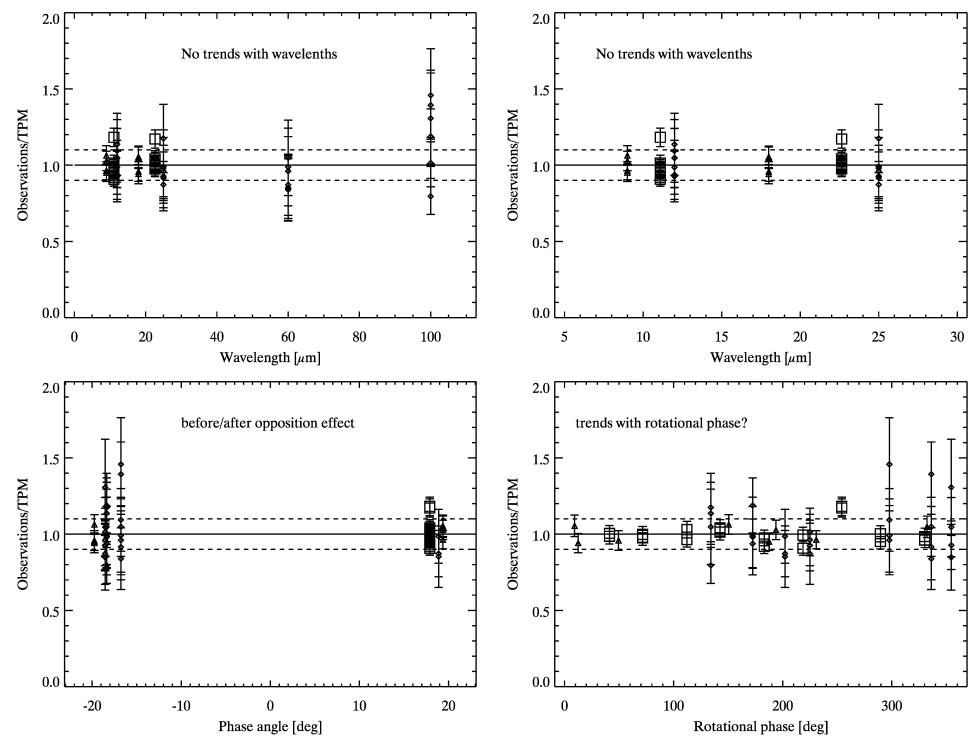

Fig. 23. O-C diagrams for thermophysical model of (478) Tergeste, using SAGE model 2. There are no trends with wavelength or preand post-opposition asymmetry. The two outliers at rotational phase 250 deg might be an indication for a small-scale shape problem, but could also be connected to a wrong flux (single WISE W3/W4 epoch where a bright background source might have influenced the photometry). For best fitting thermal parameters see Table 7 .

Some of them may prove to be good candidates for secondary calibrators for infrared observatories like ALMA, APEX, or IRAM (Müller \& Lagerros 2002) as their infrared flux is only weakly and slowly variable (although in a predictable way), which are desirable features of calibrator asteroids.

Cases like 227, 478, and 487 add support to the suggestion of Harris \& Drube (2016) that slowly rotating asteroids have higher thermal inertia values, but a larger sample is still needed. Our modelled targets applied in careful thermophysical modelling show best fitting values from 50 to 125 SI units, which seems to fit the trend to higher values of thermal inertia for rotation periods longer than $10 \mathrm{~h}$ (see Fig. 5 in Harris \& Drube 2016). With slower rotation, the heat penetrates deeper to more compact subregolith layers with substantially higher density and thermal conductivity, which both seem to rapidly grow with depth. Thermal inertia appears to grow by a factor of 10 (main belt asteroids) and 20 (near-Earth objects) with a depth of just $10 \mathrm{~cm}$ (Harris \& Drube 2016). Alternatively, the growth observed here might also be related to the objects' sizes: a low thermal inertia of 15 has been found for large (fine-grained regolith covered) asteroids with sizes much larger than $100 \mathrm{~km}$, but we are looking here at objects below or close to $100 \mathrm{~km}$. They might have less low-conductivity material on the surface, due to reduced gravity. Our future works are going to provide thermal inertia values for a larger sample of slow-rotators, a highly needed input for further studies of subsurface layers of asteroids. 

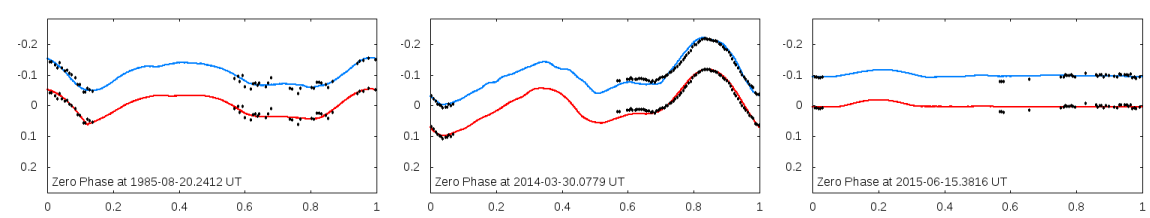

Fig. 24. Convex (upper curve) and non-convex (lower curve) model lightcurves of (487) Venetia fitted to the data from various apparitions (black points).
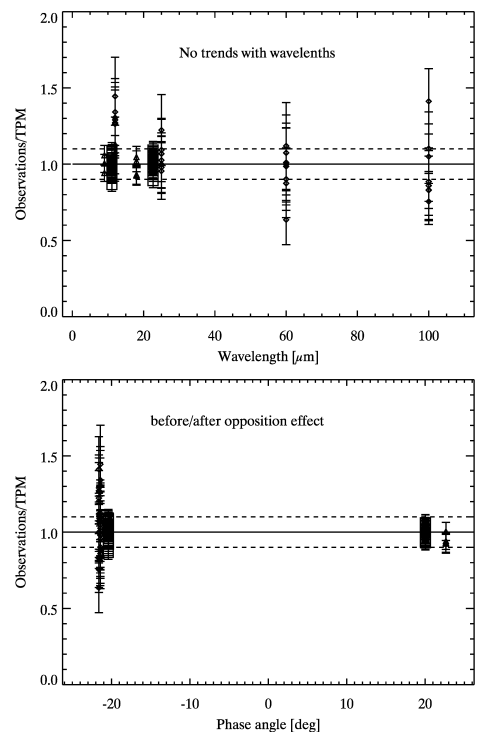
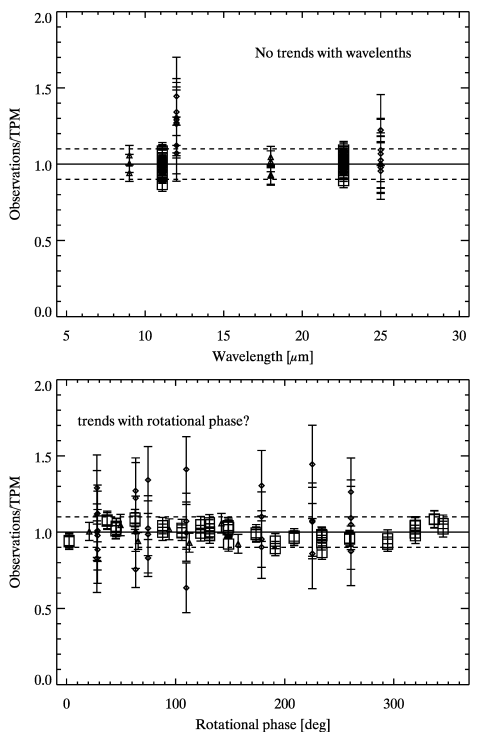

Fig. 25. O-C diagrams for the thermophysical model of (487) Venetia using SAGE model 2. There are no trends with wavelength or pre- and post-opposition asymmetry, but some trends with rotation can be noticed in the WISE data (box symbol). These data cover the object's full rotation during two separate epochs in January and July 2010, and residual trends can only be explained by shape effects. For the best fitting thermal parameters see Table 7.
Acknowledgements. This work was supported by grant No. 2014/13/D/ST9/ 01818 from the National Science Centre, Poland. The research leading to these results has received funding from the European Union's Horizon 2020 Research and Innovation Programme, under Grant Agreement No. 687378. V.K. was supported by the grant from the Slovak Research and Development Agency with number APVV-15-0458. The Joan Oró Telescope (TJO) of the Montsec Astronomical Observatory (OAdM) is owned by the Catalan Government and is operated by the Institute for Space Studies of Catalonia (IEEC). The $0.82 \mathrm{~m}$ IAC80 Telescope is operated on the island of Tenerife by the Instituto de Astrofisica de Canarias in the Spanish Observatorio del Teide. Based on observations obtained with the SARA Observatory $1.0 \mathrm{~m}$ Jacobus Kapteyn Telescope at ORM, and $0.6 \mathrm{~m}$ telescope at CTIO, which are owned and operated by the Southeastern Association for Research in Astronomy (http://saraobservatory.org).

\section{References}

Alkema, M. S. 2013, Minor Planet Bulletin, 40, 133

Bartczak, P., \& Dudziński, G. 2017, MNRAS, accepted

Bartczak, P., Michałowski, T., Santana-Ros, T., \& Dudziński, G. 2014, MNRAS, 443, 1802

Barucci, M. A., di Martino, M., \& Fulchignoni, M. 1992, AJ, 103, 1679

Behrend, R., et al. 2014 http://obswww.unige.ch/ behrend/page_cou . html

Bembrick, C., Allen, B., \& Richards, T. 2006, Minor Planet Bulletin, 33, 42

Bottke, Jr., W. F., Vokrouhlický, D., Rubincam, D. P., \& Nesvorný, D. 2006, Ann. Rev. Earth Planet. Sci., 34, 157

Brinsfield, J. W. 2009, Minor Planet Bulletin, 36, 64

Buchheim, R. K. 2007, Minor Planet Bulletin, 34, 68

Carry, B. 2012, Planet. Space Sci., 73, 98

Carry, B., Kaasalainen, M., Merline, W. J., et al. 2012, Planet. Space Sci., 66, 200

Cellino, A., Hestroffer, D., Tanga, P., Mottola, S., \& Dell'Oro, A. 2009, A\&A, 506, 935

Cibulková, H., Ďurech, J., Vokrouhlický, D., Kaasalainen, M., \& Oszkiewicz, D. A. 2016, A\&A, 596, A57

Delbo, M., Mueller, M., Emery, J. P., Rozitis, B., \& Capria, M. T. 2015, Asteroids IV, eds. P. Michel, F. E. DeMeo, \& W. F. Bottke, 107

Ditteon, R., \& Hawkins, S. 2007, Minor Planet Bulletin, 34, 59

Dunham, D. W., Herald, D., Frappa, E., et al. 2016, NASA Planetary Data System, 243
Durech, J., Vokrouhlický, D., Kaasalainen, M., et al. 2008, A\&A, 489, L25 Durech, J., Kaasalainen, M., Herald, D., et al. 2011, Icarus, 214, 652 Durech, J., Hanuš, J., Oszkiewicz, D., \& Vančo, R. 2016, A\&A, 587, A48 Erikson, A., Mottola, S., Lagerros, J. S. V., et al. 2000, Icarus, 147, 487 Ferrero, A. 2014, Minor Planet Bulletin, 41, 184

Hanuš, J., Ďurech, J., Brož, M., et al. 2011, A\&A, 530, A134

Hanuš, J., Ďurech, J., Brož, M., et al. 2013, A\&A, 551, A67

Hanuš, J., Ďurech, J., Oszkiewicz, D. A., et al. 2016, A\&A, 586, A108

Hanuš, J., Viikinkoski, M., Marchis, F., et al. 2017, A\&A, 601, A114

Harris, A. W., \& Drube, L. 2016, ApJ, 832, 127

Harris, A. W., \& Young, J. W. 1989, Icarus, 81, 314

Holsapple, K. A. 2007, Icarus, 187, 500

Johansen, A., \& Lacerda, P. 2010, MNRAS, 404, 475

Kaasalainen, M., \& Torppa, J. 2001, Icarus, 153, 24

Kaasalainen, M., Torppa, J., \& Muinonen, K. 2001, Icarus, 153, 37

Kaasalainen, M., Ďurech, J., Warner, B. D., Krugly, Y. N., \& Gaftonyuk, N. M. 2007, Nature, 446, 420

Kryszczyńska, A., Colas, F., Polińska, M., et al. 2012, A\&A, 546, A72

Lagerros, J. S. V. 1996, A\&A, 310, 1011

Lagerros, J. S. V. 1997, A\&A, 325, 1226

Lagerros, J. S. V. 1998, A\&A, 332, 1123

Lowry, S. C., Fitzsimmons, A., Pravec, P., et al. 2007, Science, 316, 272

Lowry, S. C., Weissman, P. R., Duddy, S. R., et al. 2014, A\&A, 562, A48

Mainzer, A., Grav, T., Masiero, J., et al. 2011, ApJ, 741, 90

Marciniak, A., Bartczak, P., Santana-Ros, T., et al. 2012, A\&A, 545, A131

Marciniak, A., Pilcher, F., Santana-Ros, T., Oszkiewicz, D., \& Kankiewicz, P. 2014, in Asteroids, Comets, Meteors 2014, eds. K. Muinonen, A. Penttilä, M. Granvik, et al., 333

Marciniak, A., Pilcher, F., Oszkiewicz, D., et al. 2015, Planet. Space Sci., 118, 256

Marciniak, A., Pilcher, F., Oszkiewicz, D., et al. 2016, in 37th Meeting of the Polish Astronomical Society, eds. A. Różańska, \& M. Bejger, 3, 84

Marsset, M., Carry, B., Dumas, C., et al. 2017, A\&A, 604, A64

Masiero, J. R., Mainzer, A. K., Grav, T., et al. 2011, ApJ, 741, 68

Menke, J., Cooney, W., Gross, J., Terrell, D., \& Higgins, D. 2008, Minor Planet Bulletin, 35, 155

Molnár, L., Pál, A., Sárneczky, K., et al. 2017, ApJS, submitted [arXiv: 1706.06056]

Müller, T. G., \& Lagerros, J. S. V. 1998, A\&A, 338, 340

Müller, T. G., \& Lagerros, J. S. V. 2002, A\&A, 381, 324

Müller, T., Balog, Z., Nielbock, M., et al. 2014a, Exp. Astron., 37, 253

Müller, T. G., Hasegawa, S., \& Usui, F. 2014b, PASJ, 66, 52 
A. Marciniak et al.: Long-period and low-amplitude asteroids

Neely, A. W. 1992, Minor Planet Bulletin, 19, 31

Pilcher, F. 2013, Minor Planet Bulletin, 40, 85

Pilcher, F., \& Alkema, M. S. 2014a, Minor Planet Bulletin, 41, 188

Pilcher, F., \& Alkema, M. S. 2014b, Minor Planet Bulletin, 41, 233

Pravec, P., Harris, A. W., Kušnirák, P., Galád, A., \& Hornoch, K. 2012, Icarus 221,365

Pray, D. P. 2006, Minor Planet Bulletin, 33, 4

Robinson, L. E. 2002, Minor Planet Bulletin, 29, 37

Rubincam, D. P. 2000, Icarus, 148, 2

Sada, P. V. 2004, Minor Planet Bulletin, 31, 22

Santana-Ros, T., Bartczak, P., Michałowski, T., Tanga, P., \& Cellino, A. 2015, MNRAS, 450, 333

Schober, H. J., Erikson, A., Hahn, G., et al. 1994, A\&AS, 105

Shevchenko, V. G., Chernyi, V. G., Kruglyi, I. N., et al. 1992, Icarus, 100, 295

Slivan, S. M. 2002, Nature, 419, 49

Stephens, R. D. 2005, Minor Planet Bulletin, 32, 27

Stephens, R. D. 2010, Minor Planet Bulletin, 37, 122

Stephens, R. D. 2013, Minor Planet Bulletin, 40, 34
Szabó, R., Pál, A., Sárneczky, K., et al. 2016, A\&A, 596, A40

Szabó, G. M., Pál, A., Kiss, C., et al. 2017, A\&A, 599, A44

Takeda, T., \& Ohtsuki, K. 2009, Icarus, 202, 514

Tedesco, E. F., Noah, P. V., Noah, M., \& Price, S. D. 2004, NASA Planetary Data System, 12

Timerson, B., Durech, J., Aguirre, S., et al. 2009, Minor Planet Bulletin, 36, 98 Tungalag, N., Shevchenko, V. G., \& Lupishko, D. F. 2002, Kinematika i Fizika Nebesnykh Tel, 18, 508

Usui, F., Kuroda, D., Müller, T. G., et al. 2011, PASJ, 63, 1117

Viikinkoski, M., Kaasalainen, M., \& Durech, J. 2015, A\&A, 576, A8

Vokrouhlický, D., Bottke, W. F., Chesley, S. R., Scheeres, D. J., \& Statler, T. S. 2015, Asteroids IV, eds. P. Michel, F. E. DeMeo, \& W. F. Bottke (Tucson: University of Arizona Press), 509

Warner, B. D., Harris, A. W., \& Pravec, P. 2009, Icarus, 202, 134

Warner, B. D., Sada, P. V., Pollock, J., et al. 2010, Minor Planet Bulletin, 37, 139

Weidenschilling, S. J., Chapman, C. R., Davis, D. R., Greenberg, R., \& Levy, D. H. 1990, Icarus, 86,402 


\section{Appendix A: Additional tables and figures}

Observing runs details (Table A.1) and composite lightcurves of asteroids with new period determinations (Figs. A.1-A.5) and asteroids with spin and shape models presented here (Figs. A.7-A.24).

Table A.1. Observation details: mid-time observing date, ecliptic longitude of the target, sun-target-observer phase angle, duration of the observing run, brightness scatter, observer, and site name.

\begin{tabular}{|c|c|c|c|c|c|c|}
\hline Date & $\begin{array}{l}\lambda \\
{[\mathrm{deg}]}\end{array}$ & $\begin{array}{l}\text { Phase angle } \\
\text { [deg] }\end{array}$ & $\begin{array}{l}\text { Duration } \\
\text { [hours] }\end{array}$ & $\begin{array}{l}\sigma \\
{[\mathrm{mag}]}\end{array}$ & Observer & Site \\
\hline \multicolumn{7}{|l|}{ (551) Ortrud } \\
\hline 2016 Aug. 31.0 & 32.9 & 17.5 & 4.7 & 0.005 & K. Żukowski & Borowiec \\
\hline 2016 Sep. 02.0 & 33.0 & 17.1 & 4.8 & 0.008 & A. Marciniak & Borowiec \\
\hline 2016 Sep. 03.0 & 33.0 & 16.8 & 3.7 & 0.008 & R. Hirsch & Borowiec \\
\hline 2016 Sep. 05.4 & 32.9 & 16.3 & 3.6 & 0.011 & F. Pilcher & Organ Mesa Obs. \\
\hline 2016 Sep. 09.0 & 32.8 & 15.4 & 4.8 & 0.014 & M. Butkiewicz Bạk & Borowiec \\
\hline 2016 Sep. 13.4 & 32.6 & 14.1 & 5.3 & 0.006 & F. Pilcher & Organ Mesa Obs. \\
\hline 2016 Sep. 14.4 & 32.5 & 13.8 & 7.2 & 0.007 & F. Pilcher & Organ Mesa Obs. \\
\hline 2016 Sep. 15.3 & 32.4 & 13.6 & 2.8 & 0.013 & F. Pilcher & Organ Mesa Obs. \\
\hline 2016 Oct. 01.3 & 30.4 & 7.8 & 8.4 & 0.005 & F. Pilcher & Organ Mesa Obs. \\
\hline 2016 Nov. 18.2 & 21.4 & 12.2 & 5.7 & 0.016 & T. Polakis & Tempe \\
\hline 2016 Nov. 19.2 & 21.3 & 12.5 & 5.7 & 0.016 & T. Polakis & Tempe \\
\hline 2016 Nov. 22.2 & 21.0 & 13.5 & 5.9 & 0.017 & T. Polakis & Tempe \\
\hline 2016 Nov. 23.2 & 20.9 & 13.8 & 5.2 & 0.016 & T. Polakis & Tempe \\
\hline 2016 Nov. 24.2 & 20.9 & 14.1 & 6.0 & 0.018 & T. Polakis & Tempe \\
\hline 2016 Nov. 25.2 & 20.8 & 14.4 & $\begin{array}{l}4.1 \\
77.9 \text { total }\end{array}$ & 0.016 & T. Polakis & Tempe \\
\hline \multicolumn{7}{|l|}{ (581) Tauntonia } \\
\hline 2016 Jan. 28.3 & 120.2 & 4.2 & 1.7 & 0.002 & K. Kamiński & Winer Obs. \\
\hline 2016 Jan. 29.4 & 119.9 & 4.5 & 7.2 & 0.006 & K. Kamiński & Winer Obs. \\
\hline 2016 Feb. 22.4 & 115.6 & 11.6 & 6.0 & 0.011 & K. Kamiński & Winer Obs. \\
\hline 2016 Feb. 24.2 & 115.4 & 12.1 & 5.7 & 0.012 & K. Kamiński & Winer Obs. \\
\hline 2016 Feb. 26.3 & 115.2 & 12.6 & 7.6 & 0.006 & K. Kamiński & Winer Obs. \\
\hline 2016 Mar. 23.9 & 114.5 & 17.5 & 5.8 & 0.007 & - & Montsec Obs. \\
\hline 2016 Mar. 24.9 & 114.5 & 17.6 & 5.8 & 0.007 & - & Montsec Obs. \\
\hline 2016 Mar. 27.9 & 114.7 & 17.9 & 5.8 & 0.005 & - & Montsec Obs. \\
\hline \multirow[t]{2}{*}{ 2016 Mar. 31.9} & 115.1 & 18.2 & 3.4 & 0.006 & - & Montsec Obs. \\
\hline & & & 49.0 total & & & \\
\hline \multicolumn{7}{|c|}{ (830) Petropolitana } \\
\hline 2017 Mar. 01.4 & 178.0 & 5.1 & 7.6 & 0.012 & T. Polakis & Tempe \\
\hline 2017 Mar. 02.4 & 177.8 & 4.8 & 7.5 & 0.013 & T. Polakis & Tempe \\
\hline 2017 Mar. 04.4 & 177.5 & 4.1 & 7.6 & 0.011 & T. Polakis & Tempe \\
\hline 2017 Mar. 06.4 & 177.1 & 3.3 & 7.5 & 0.010 & T. Polakis & Tempe \\
\hline 2017 Mar. 07.4 & 176.9 & 3.0 & 7.5 & 0.009 & T. Polakis & Tempe \\
\hline 2017 Mar. 08.4 & 176.7 & 2.6 & 7.2 & 0.010 & T. Polakis & Tempe \\
\hline 2017 Mar. 09.4 & 176.5 & 2.3 & 6.9 & 0.014 & T. Polakis & Tempe \\
\hline 2017 Mar. 10.4 & 176.3 & 1.9 & 6.9 & 0.011 & T. Polakis & Tempe \\
\hline 2017 Apr. 14.2 & 170.2 & 9.9 & 6.8 & 0.013 & T. Polakis & Tempe \\
\hline 2017 Apr. 15.2 & 170.1 & 10.2 & 6.6 & 0.011 & T. Polakis & Tempe \\
\hline 2017 Apr. 18.3 & 169.8 & 11.0 & 3.0 & 0.017 & T. Polakis & Tempe \\
\hline 2017 Apr. 19.2 & 169.7 & 11.2 & 6.1 & 0.017 & T. Polakis & Tempe \\
\hline 2017 Apr. 20.2 & 169.6 & 11.5 & 6.1 & 0.019 & T. Polakis & Tempe \\
\hline 2017 Apr. 21.2 & 169.5 & 11.7 & 5.7 & 0.019 & T. Polakis & Tempe \\
\hline 2017 Apr. 22.2 & 169.4 & 12.0 & 5.9 & 0.016 & T. Polakis & Tempe \\
\hline 2017 Apr. 23.3 & 169.3 & 12.2 & 4.1 & 0.017 & T. Polakis & Tempe \\
\hline \multirow[t]{2}{*}{2017 Apr. 24.2} & 169.2 & 12.5 & 5.2 & 0.023 & T. Polakis & Tempe \\
\hline & & & 108.2 total & & & \\
\hline
\end{tabular}

Notes. See Table 1 for telescope and site details. 
Table A.1. continued.

\begin{tabular}{|c|c|c|c|c|c|c|}
\hline Date & $\begin{array}{l}\lambda \\
{[\mathrm{deg}]}\end{array}$ & $\begin{array}{l}\text { Phase angle } \\
\text { [deg] }\end{array}$ & $\begin{array}{l}\text { Duration } \\
\text { [hours] }\end{array}$ & $\begin{array}{l}\sigma \\
{[\mathrm{mag}]}\end{array}$ & Observer & Site \\
\hline \multicolumn{7}{|l|}{ (923) Herluga } \\
\hline 2016 Jul. 26.0 & 330.2 & 13.1 & 4.2 & 0.007 & - & Montsec Obs. \\
\hline 2016 Jul. 27.0 & 330.0 & 12.8 & 3.2 & 0.006 & - & Montsec Obs. \\
\hline 2016 Jul. 28.0 & 329.8 & 12.6 & 3.2 & 0.005 & - & Montsec Obs. \\
\hline 2016 Aug. 03.0 & 328.7 & 10.8 & 3.8 & 0.003 & - & Montsec Obs. \\
\hline 2016 Aug. 04.0 & 328.5 & 10.6 & 3.6 & 0.019 & - & Montsec Obs. \\
\hline 2016 Aug. 08.0 & 327.6 & 9.6 & 4.4 & 0.003 & - & Montsec Obs. \\
\hline 2016 Aug. 11.0 & 326.9 & 9.0 & 7.3 & 0.006 & - & Montsec Obs. \\
\hline 2016 Aug. 15.1 & 326.0 & 8.4 & 7.6 & 0.002 & S. Geier & JKT, ORM \\
\hline 2016 Aug. 19.9 & 324.8 & 8.3 & 6.7 & 0.022 & R. Hirsch & Borowiec \\
\hline 2016 Aug. 22.9 & 324.0 & 8.5 & 7.0 & 0.014 & K. Żukowski & Borowiec \\
\hline 2016 Sep. 06.9 & 320.7 & 12.2 & 5.7 & 0.008 & A. Marciniak & Borowiec \\
\hline 2016 Sep. 10.9 & 320.0 & 13.6 & $\begin{array}{l}5.3 \\
62.0 \text { total }\end{array}$ & 0.013 & A. Marciniak & Borowiec \\
\hline \multicolumn{7}{|l|}{ (932) Hooveria } \\
\hline 2016 Nov. 18.3 & 58.8 & 4.6 & 10.2 & 0.007 & T. Polakis & Tempe \\
\hline 2016 Nov. 19.3 & 58.6 & 4.5 & 10.2 & 0.006 & T. Polakis & Tempe \\
\hline 2016 Nov. 22.3 & 57.8 & 4.7 & 10.2 & 0.005 & T. Polakis & Tempe \\
\hline 2016 Nov. 23.3 & 57.5 & 4.9 & 11.9 & 0.007 & T. Polakis & Tempe \\
\hline 2016 Nov. 24.2 & 57.3 & 5.1 & 7.5 & 0.007 & T. Polakis & Tempe \\
\hline 2016 Nov. 25.3 & 57.0 & 5.4 & 10.1 & 0.006 & T. Polakis & Tempe \\
\hline 2016 Nov. 26.4 & 56.8 & 5.7 & 2.1 & 0.010 & T. Polakis & Tempe \\
\hline 2016 Nov. 30.2 & 55.8 & 7.2 & 8.7 & 0.007 & T. Polakis & Tempe \\
\hline 2016 Dec. 01.3 & 55.6 & 7.7 & 6.9 & 0.007 & T. Polakis & Tempe \\
\hline 2016 Dec. 03.3 & 55.1 & 8.5 & 6.3 & 0.008 & T. Polakis & Tempe \\
\hline 2016 Dec. 04.3 & 54.9 & 9.0 & 9.2 & 0.007 & T. Polakis & Tempe \\
\hline 2016 Dec. 05.3 & 54.7 & 9.4 & 9.2 & 0.007 & T. Polakis & Tempe \\
\hline 2016 Dec. 09.2 & 53.9 & 11.2 & 9.0 & 0.008 & T. Polakis & Tempe \\
\hline 2016 Dec. 18.2 & 52.5 & 15.0 & 7.4 & 0.009 & T. Polakis & Tempe \\
\hline 2016 Dec. 19.2 & 52.4 & 15.4 & 7.4 & 0.009 & T. Polakis & Tempe \\
\hline \multirow[t]{2}{*}{2016 Dec. 20.1} & 52.3 & 15.8 & 3.3 & 0.015 & T. Polakis & Tempe \\
\hline & & & 129.6 total & & & \\
\hline \multicolumn{7}{|l|}{ (995) Sternberga } \\
\hline 2016 May 04.4 & 265.0 & 15.1 & 3.9 & 0.006 & B. Skiff & Lowell Obs. \\
\hline 2016 May 11.2 & 264.3 & 13.0 & 2.0 & 0.003 & S. Geier & Teide \\
\hline 2016 May 24.0 & 262.4 & 8.7 & 2.1 & 0.028 & V. Kudak & Derenivka \\
\hline 2016 Jun. 02.2 & 260.4 & 6.0 & 0.8 & 0.006 & B. Skiff & Lowell Obs. \\
\hline 2016 Jun. 03.3 & 260.1 & 5.8 & 7.0 & 0.004 & B. Skiff & Lowell Obs. \\
\hline 2016 Jun. 04.3 & 259.9 & 5.6 & 7.1 & 0.004 & B. Skiff & Lowell Obs. \\
\hline 2016 Jun. 05.3 & 259.7 & 5.5 & 7.0 & 0.005 & B. Skiff & Lowell Obs. \\
\hline 2016 Jun. 06.3 & 259.4 & 5.4 & 7.0 & 0.006 & B. Skiff & Lowell Obs. \\
\hline 2016 Jun. 07.3 & 259.2 & 5.3 & 4.0 & 0.007 & T. Polakis & Tempe \\
\hline 2016 Jun. 08.1 & 259.0 & 5.3 & 4.1 & 0.020 & R. Duffard & La Sagra \\
\hline 2016 Jun. 08.4 & 259.0 & 5.3 & 4.2 & 0.008 & T. Polakis & Tempe \\
\hline 2016 Jun. 09.1 & 258.8 & 5.3 & 3.4 & 0.016 & R. Duffard & La Sagra \\
\hline 2016 Jun. 10.1 & 258.5 & 5.4 & 3.7 & 0.014 & R. Duffard & La Sagra \\
\hline 2016 Jun. 12.1 & 258.0 & 5.6 & 2.8 & 0.018 & R. Duffard & La Sagra \\
\hline 2016 Jun. 13.1 & 258.0 & 5.8 & 3.2 & 0.021 & R. Duffard & La Sagra \\
\hline 2016 Jun. 13.3 & 257.7 & 5.8 & 6.7 & 0.005 & B. Skiff & Lowell Obs. \\
\hline 2016 Jun. 30.9 & 253.8 & 11.6 & 3.3 & 0.004 & - & Montsec Obs. \\
\hline 2016 Jul. 06.0 & 253.0 & 13.5 & 3.0 & 0.008 & - & Montsec Obs. \\
\hline 2016 Jul. 06.9 & 252.8 & 13.8 & 2.4 & 0.004 & S. Fauvaud & Bardon Obs. \\
\hline 2016 Jul. 07.0 & 252.8 & 13.8 & 3.0 & 0.008 & - & Montsec Obs. \\
\hline 2016 Jul. 08.0 & 252.6 & 14.2 & 1.9 & 0.005 & S. Fauvaud & Bardon Obs. \\
\hline 2016 Jul. 08.9 & 252.5 & 14.5 & 2.8 & 0.005 & S. Fauvaud & Bardon Obs. \\
\hline 2016 Jul. 09.0 & 252.5 & 14.5 & 3.2 & 0.006 & - & Montsec Obs. \\
\hline \multirow[t]{2}{*}{2016 Jul. 10.0} & 252.4 & 14.9 & 3.9 & 0.005 & S. Fauvaud & Bardon Obs. \\
\hline & & & 92.5 total & & & \\
\hline
\end{tabular}


Table A.1. continued.

\begin{tabular}{|c|c|c|c|c|c|c|}
\hline Date & $\begin{array}{l}\lambda \\
{[\mathrm{deg}]}\end{array}$ & $\begin{array}{l}\text { Phase angle } \\
\text { [deg] }\end{array}$ & $\begin{array}{l}\text { Duration } \\
\text { [hours] }\end{array}$ & $\begin{array}{l}\sigma \\
{[\mathrm{mag}]}\end{array}$ & Observer & Site \\
\hline \multicolumn{7}{|l|}{ (159) Aemilia } \\
\hline 2005 Jul. 03.1 & 317.6 & 10.2 & 2.6 & 0.013 & L. Bernasconi & Obs. des Engarouines \\
\hline 2005 Jul. 09.0 & 316.9 & 8.5 & 3.5 & 0.011 & L. Bernasconi & Obs. des Engarouines \\
\hline 2005 Jul. 11.0 & 316.6 & 8.0 & 4.3 & 0.018 & L. Bernasconi & Obs. des Engarouines \\
\hline 2005 Aug. 07.0 & 311.8 & 0.8 & 5.6 & 0.014 & L. Bernasconi & Obs. des Engarouines \\
\hline 2005 Aug. 09.0 & 311.4 & 1.5 & 5.9 & 0.015 & L. Bernasconi & Obs. des Engarouines \\
\hline 2013 Dec. 28.2 & 180.3 & 19.9 & 3.5 & 0.004 & R. Hirsch & Borowiec \\
\hline 2014 Jan. 17.4 & 182.6 & 18.0 & 6.0 & 0.006 & F. Pilcher & Organ Mesa Obs. \\
\hline 2014 Jan. 25.1 & 182.9 & 16.7 & 5.5 & 0.006 & K. Sobkowiak & Borowiec \\
\hline 2014 Jan. 28.4 & 182.9 & 16.1 & 6.9 & 0.003 & F. Pilcher & Organ Mesa Obs. \\
\hline 2014 Feb. 04.1 & 182.7 & 14.5 & 6.5 & 0.005 & A. Marciniak & Borowiec \\
\hline 2014 Feb. 09.4 & 182.4 & 13.1 & 7.5 & 0.006 & F. Pilcher & Organ Mesa Obs. \\
\hline 2014 Feb. 13.1 & 182.0 & 12.0 & 7.5 & 0.005 & A. Marciniak & Borowiec \\
\hline 2014 Feb. 21.1 & 181.0 & 9.5 & 6.2 & 0.005 & I. Konstanciak & Borowiec \\
\hline 2014 Mar. 29.9 & 173.9 & 5.5 & 7.0 & 0.004 & A. Marciniak & Borowiec \\
\hline 2015 Apr. 29.4 & 259.0 & 11.4 & 5.0 & 0.007 & K. Kamiński & Winer Obs. \\
\hline 2015 May 19.9 & 256.1 & 5.6 & 2.9 & 0.005 & M. Żejmo & Adiyaman Obs. \\
\hline 2015 May 30.3 & 254.1 & 2.8 & 7.6 & 0.010 & K. Kamiński & Winer Obs. \\
\hline 2015 May 30.4 & 254.1 & 2.8 & 6.4 & 0.008 & F. Pilcher & Organ Mesa Obs. \\
\hline 2015 Jun. 12.9 & 251.5 & 3.8 & 4.2 & 0.007 & M. Żejmo & Adiyaman Obs. \\
\hline 2015 Jun. 18.0 & 250.6 & 5.2 & 4.9 & 0.005 & - & Montsec Obs. \\
\hline 2015 Jun. 20.3 & 250.2 & 5.9 & 4.3 & 0.004 & F. Pilcher & Organ Mesa Obs. \\
\hline 2015 Jun. 22.2 & 249.8 & 6.4 & 5.7 & 0.005 & F. Pilcher & Organ Mesa Obs. \\
\hline 2015 Jun. 25.0 & 249.4 & 7.2 & 4.4 & 0.003 & - & Montsec Obs. \\
\hline 2015 Jul. 02.2 & 248.3 & 9.3 & 5.1 & 0.010 & F. Pilcher & Organ Mesa Obs. \\
\hline 2015 Jul. 07.2 & 247.8 & 10.6 & $\begin{array}{l}4.7 \\
133.7 \text { total }\end{array}$ & 0.006 & F. Pilcher & Organ Mesa Obs. \\
\hline \multicolumn{7}{|l|}{ (227) Philosophia } \\
\hline 2006 Nov. 09.1 & 43.6 & 3.2 & 4.2 & 0.030 & R. Ditteon & Oakley Obs. \\
\hline 2006 Nov. 09.3 & 43.6 & 3.2 & 4.3 & 0.035 & R. Ditteon & Oakley Obs. \\
\hline 2006 Nov. 10.1 & 43.4 & 3.3 & 5.1 & 0.028 & R. Ditteon & Oakley Obs. \\
\hline 2006 Nov. 10.3 & 43.4 & 3.3 & 3.3 & 0.038 & R. Ditteon & Oakley Obs. \\
\hline 2006 Nov. 15.0 & 42.5 & 4.0 & 9.6 & 0.011 & P. Antonini & Obs. Hauts Patys \\
\hline 2006 Nov. 29.9 & 39.9 & 7.5 & 7.2 & 0.020 & P. Antonini & Obs. Hauts Patys \\
\hline 2006 Dec. 27.9 & 37.3 & 12.9 & 8.4 & 0.026 & P. Antonini & Obs. Hauts Patys \\
\hline 2015 Apr. 15.4 & 223.0 & 8.5 & 6.0 & 0.004 & K. Kamiński & Winer Obs. \\
\hline 2015 Apr. 17.4 & 222.7 & 7.9 & 6.0 & 0.003 & K. Kamiński & Winer Obs. \\
\hline 2015 Apr. 19.4 & 222.3 & 7.4 & 6.0 & 0.003 & K. Kamiński & Winer Obs. \\
\hline 2015 Apr. 30.3 & 220.2 & 5.6 & 5.0 & 0.004 & K. Kamiński & Winer Obs. \\
\hline 2015 May 06.3 & 219.0 & 6.1 & 5.3 & 0.009 & K. Kamiński & Winer Obs. \\
\hline 2015 May 10.3 & 218.2 & 7.0 & 5.8 & 0.003 & K. Kamiński & Winer Obs. \\
\hline 2015 May 12.3 & 217.8 & 7.6 & 3.3 & 0.008 & K. Kamiński & Winer Obs. \\
\hline 2015 May 13.3 & 217.6 & 7.9 & 5.3 & 0.004 & K. Kamiński & Winer Obs. \\
\hline 2015 May 14.3 & 217.5 & 8.2 & 5.0 & 0.007 & K. Kamiński & Winer Obs. \\
\hline 2015 May 28.2 & 215.4 & 12.8 & 4.1 & 0.003 & K. Kamiński & Winer Obs. \\
\hline 2015 Jun. 29.9 & 215.1 & 20.8 & 2.5 & 0.005 & A. Marciniak & Teide Obs. \\
\hline 2016 Jul. 07.4 & 337.6 & 15.3 & 3.1 & 0.003 & D. Oszkiewicz, B. Skiff & Lowell Obs. \\
\hline 2016 Jul. 14.4 & 337.2 & 13.6 & 4.7 & 0.004 & D. Oszkiewicz, B. Skiff & Lowell Obs. \\
\hline 2016 Jul. 17.4 & 337.0 & 12.8 & 5.1 & 0.007 & D. Oszkiewicz, B. Skiff & Lowell Obs. \\
\hline 2016 Jul. 21.3 & 336.6 & 11.7 & 4.7 & 0.015 & D. Oszkiewicz & Cerro Tololo \\
\hline 2016 Jul. 25.1 & 336.1 & 10.5 & 5.7 & 0.004 & A. Marciniak & Teide Obs. \\
\hline 2016 Jul. 28.4 & 335.6 & 9.5 & 4.7 & 0.005 & D. Oszkiewicz, B. Skiff & Lowell Obs. \\
\hline
\end{tabular}


Table A.1. continued.

\begin{tabular}{|c|c|c|c|c|c|c|}
\hline Date & $\begin{array}{l}\lambda \\
{[\mathrm{deg}]}\end{array}$ & $\begin{array}{l}\text { Phase angle } \\
\text { [deg] }\end{array}$ & $\begin{array}{l}\text { Duration } \\
\text { [hours] }\end{array}$ & $\begin{array}{l}\sigma \\
{[\mathrm{mag}]}\end{array}$ & Observer & Site \\
\hline 2016 Aug. 12.3 & 333.0 & 4.2 & 7.2 & 0.004 & B. Skiff & Lowell Obs. \\
\hline 2016 Aug. 14.3 & 332.6 & 3.5 & 7.7 & 0.006 & B. Skiff & Lowell Obs. \\
\hline 2016 Aug. 15.3 & 332.4 & 3.1 & 7.7 & 0.008 & F. Pilcher & Organ Mesa Obs. \\
\hline 2016 Aug. 15.3 & 332.4 & 3.1 & 7.0 & 0.005 & B. Skiff & Lowell Obs. \\
\hline 2016 Aug. 16.3 & 332.2 & 2.7 & 7.3 & 0.008 & F. Pilcher & Organ Mesa Obs. \\
\hline 2016 Aug. 21.0 & 331.3 & 1.0 & 4.3 & 0.009 & - & Montsec Obs. \\
\hline 2016 Aug. 23.3 & 330.8 & 0.3 & 6.4 & 0.011 & F. Pilcher & Organ Mesa Obs. \\
\hline 2016 Aug. 26.9 & 330.1 & 1.3 & 4.2 & 0.007 & R. Hirsch & Borowiec \\
\hline 2016 Aug. 29.3 & 329.6 & 2.2 & 5.8 & 0.004 & B. Skiff & Lowell Obs. \\
\hline 2016 Sep. 04.4 & 328.4 & 4.4 & 7.4 & 0.004 & B. Skiff & Lowell Obs. \\
\hline 2016 Sep. 08.0 & 327.8 & 5.7 & 5.2 & 0.006 & - & Montsec Obs. \\
\hline 2016 Sep. 09.2 & 327.6 & 6.1 & 7.0 & 0.005 & F. Pilcher & Organ Mesa Obs. \\
\hline 2016 Sep. 09.2 & 327.6 & 6.1 & 7.3 & 0.004 & B. Skiff & Lowell Obs. \\
\hline 2016 Sep. 10.2 & 327.4 & 6.4 & 6.9 & 0.006 & F. Pilcher & Organ Mesa Obs. \\
\hline 2016 Sep. 11.2 & 327.2 & 6.8 & 6.9 & 0.006 & F. Pilcher & Organ Mesa Obs. \\
\hline 2016 Sep. 11.2 & 327.2 & 6.8 & 7.4 & 0.005 & B. Skiff & Lowell Obs. \\
\hline 2016 Sep. 17.2 & 326.3 & 8.7 & 6.6 & 0.008 & B. Skiff & Lowell Obs. \\
\hline 2016 Sep. 17.2 & 326.3 & 8.7 & 5.0 & 0.013 & F. Pilcher & Organ Mesa Obs. \\
\hline 2016 Sep. 18.2 & 326.2 & 9.0 & 6.5 & 0.009 & F. Pilcher & Organ Mesa Obs. \\
\hline 2016 Sep. 18.2 & 326.2 & 9.0 & 6.5 & 0.008 & B. Skiff & Lowell Obs. \\
\hline 2016 Sep. 19.2 & 326.1 & 9.3 & 6.5 & 0.009 & F. Pilcher & Organ Mesa Obs. \\
\hline 2016 Sep. 19.2 & 326.1 & 9.3 & 6.5 & 0.008 & B. Skiff & Lowell Obs. \\
\hline 2016 Sep. 24.3 & 325.5 & 10.7 & 2.8 & 0.007 & B. Skiff & Lowell Obs. \\
\hline 2016 Sep. 25.2 & 325.4 & 11.0 & 5.6 & 0.006 & B. Skiff & Lowell Obs. \\
\hline 2016 Sep. 26.1 & 325.3 & 11.2 & 2.0 & 0.005 & B. Skiff & Lowell Obs. \\
\hline 2016 Oct. 02.1 & 324.8 & 12.7 & \multicolumn{3}{|l|}{284.3 total } & Lowell Obs. \\
\hline (329) Svea & & & & & & \\
\hline 2006 Jul. 24.0 & 325.6 & 11.9 & 4.3 & 0.022 & L. Bernasconi & Obs. des Engarouines \\
\hline 2006 Jul. 26.0 & 325.2 & 11.2 & 6.2 & 0.028 & L. Bernasconi & Obs. des Engarouines \\
\hline 2006 Jul. 29.0 & 324.5 & 10.2 & 5.1 & 0.022 & L. Bernasconi & Obs. des Engarouines \\
\hline 2006 Jul. 30.0 & 324.3 & 9.9 & 5.8 & 0.023 & L. Bernasconi & Obs. des Engarouines \\
\hline 2006 Aug. 21.0 & 318.9 & 7.3 & 6.4 & 0.009 & R. Poncy & Le Crès \\
\hline 2006 Aug. 22.0 & 318.7 & 7.5 & 6.2 & 0.010 & R. Poncy & Le Crès \\
\hline 2006 Aug. 27.9 & 317.3 & 9.0 & 6.2 & 0.012 & R. Poncy & Le Crès \\
\hline 2014 Jul. 30.0 & 352.9 & 17.3 & 3.2 & 0.004 & A. Marciniak & Borowiec \\
\hline 2014 Aug. 03.0 & 352.6 & 16.1 & 4.5 & 0.007 & A. Marciniak & Borowiec \\
\hline 2014 Aug. 09.0 & 351.9 & 14.0 & 5.0 & 0.010 & A. Marciniak & Borowiec \\
\hline 2014 Aug. 28.0 & 348.2 & 6.2 & 6.5 & 0.007 & A. Marciniak & Borowiec \\
\hline 2014 Sep. 04.0 & 346.5 & 3.4 & 6.5 & 0.009 & A. Marciniak & Borowiec \\
\hline 2014 Sep. 19.0 & 342.7 & 5.6 & 5.0 & 0.011 & A. Marciniak & Borowiec \\
\hline 2014 Sep. 28.8 & 340.6 & 9.8 & 3.0 & 0.017 & A. Marciniak & Borowiec \\
\hline 2014 Oct. 03.8 & 339.7 & 11.8 & 5.7 & 0.025 & K. Sobkowiak & Borowiec \\
\hline 2014 Oct. 09.8 & 338.9 & 14.0 & 2.4 & 0.006 & J. Horbowicz & Borowiec \\
\hline 2014 Oct. 10.2 & 338.9 & 14.2 & 4.7 & 0.007 & F. Pilcher & Organ Mesa Obs. \\
\hline 2014 Nov. 26.2 & 341.3 & 22.9 & 3.7 & 0.007 & K. Kamiński & Winer Obs. \\
\hline 2015 Nov. 25.1 & 110.0 & 17.8 & 6.2 & 0.009 & R. Hirsch & Borowiec \\
\hline 2015 Dec. 14.0 & 107.5 & 12.9 & 5.9 & 0.002 & - & Montsec Obs. \\
\hline 2015 Dec. 16.0 & 107.1 & 12.4 & 7.4 & 0.010 & - & Montsec Obs. \\
\hline 2015 Dec. 17.0 & 107.0 & 12.2 & 8.3 & 0.008 & - & Montsec Obs. \\
\hline 2015 Dec. 18.0 & 106.7 & 11.9 & 7.4 & 0.005 & - & Montsec Obs. \\
\hline 2015 Dec. 19.0 & 106.5 & 11.7 & 7.5 & 0.009 & - & Montsec Obs. \\
\hline 2015 Dec. 22.0 & 105.8 & 11.0 & 7.6 & 0.004 & - & Montsec Obs. \\
\hline 2015 Dec. 23.0 & 105.5 & 10.8 & 7.5 & 0.006 & - & Montsec Obs. \\
\hline 2015 Dec. 28.0 & 104.3 & 9.9 & 7.4 & 0.025 & - & Montsec Obs. \\
\hline 2015 Dec. 29.9 & 103.8 & 9.7 & 3.8 & 0.004 & - & Montsec Obs. \\
\hline
\end{tabular}


Table A.1. continued.

\begin{tabular}{|c|c|c|c|c|c|c|}
\hline Date & $\begin{array}{l}\lambda \\
{[\mathrm{deg}]}\end{array}$ & $\begin{array}{l}\text { Phase angle } \\
\text { [deg] }\end{array}$ & $\begin{array}{l}\text { Duration } \\
\text { [hours] }\end{array}$ & $\begin{array}{l}\sigma \\
{[\mathrm{mag}]}\end{array}$ & Observer & Site \\
\hline 2015 Dec. 30.9 & 103.5 & 9.6 & 4.3 & 0.002 & - & Montsec Obs. \\
\hline 2016 Jan. 24.9 & 97.4 & 13.0 & 7.4 & 0.006 & - & Montsec Obs. \\
\hline 2016 Feb. 01.9 & 96.0 & 15.2 & 7.6 & 0.005 & - & Montsec Obs. \\
\hline 2016 Mar. 02.2 & 95.4 & 21.5 & $\begin{array}{l}3.0 \\
181.7 \text { total }\end{array}$ & 0.004 & K. Kamiński & Winer Obs. \\
\hline (478) Tergeste & & & & & & \\
\hline 2005 Jul. 16.0 & 315.2 & 8.3 & 4.7 & 0.006 & L. Bernasconi & Obs. des Engarouines \\
\hline 2005 Jul. 17.0 & 315.0 & 8.1 & 5.2 & 0.021 & L. Bernasconi & Obs. des Engarouines \\
\hline 2005 Aug. 06.0 & 311.1 & 5.7 & 5.0 & 0.007 & L. Bernasconi & Obs. des Engarouines \\
\hline 2005 Aug. 08.0 & 310.7 & 5.6 & 5.8 & 0.010 & L. Bernasconi & Obs. des Engarouines \\
\hline 2005 Aug. 08.0 & 310.7 & 5.8 & 4.9 & 0.010 & R. Crippa, F. Manzini & Stazione Astro. di Sozzago \\
\hline 2005 Aug. 09.9 & 310.3 & 6.0 & 2.4 & 0.010 & R. Crippa, F. Manzini & Stazione Astro. di Sozzago \\
\hline 2005 Aug. 12.0 & 309.8 & 6.3 & 5.0 & 0.010 & L. Bernasconi & Obs. des Engarouines \\
\hline 2005 Aug. 12.9 & 309.7 & 6.5 & 4.2 & 0.006 & $\begin{array}{l}\text { R. Stoss, P. Korlevic, } \\
\text { M. Hren, } \\
\text { A. Cikota, L. Jerosimic }\end{array}$ & OAM-Mallorca \\
\hline 2005 Aug. 13.0 & 309.6 & 6.5 & 4.2 & 0.006 & R. Crippa, F. Manzini & Stazione Astro. di Sozzago \\
\hline 2005 Aug. 13.0 & 309.6 & 6.5 & 5.8 & 0.013 & L. Bernasconi & Obs. des Engarouines \\
\hline 2005 Aug. 15.0 & 309.2 & 6.8 & 3.2 & 0.007 & R. Crippa, F. Manzini & Stazione Astro. di Sozzago \\
\hline 2005 Aug. 16.0 & 309.0 & 7.0 & 3.2 & 0.007 & R. Crippa, F. Manzini & Stazione Astro. di Sozzago \\
\hline 2012 Nov. 26.2 & 108.5 & 14.7 & 1.5 & 0.006 & M. Murawiecka & Borowiec \\
\hline 2013 Feb. 15.1 & 95.7 & 16.5 & 2.8 & 0.003 & F. Pilcher & Organ Mesa Obs. \\
\hline 2013 Feb. 16.1 & 95.6 & 16.7 & 4.1 & 0.003 & F. Pilcher & Organ Mesa Obs. \\
\hline 2013 Feb. 22.1 & 95.6 & 17.9 & 4.3 & 0.008 & F. Pilcher & Organ Mesa Obs. \\
\hline 2013 Feb. 24.1 & 95.7 & 18.3 & 4.2 & 0.005 & F. Pilcher & Organ Mesa Obs. \\
\hline 2013 Mar. 12.1 & 97.0 & 20.3 & 2.7 & 0.003 & F. Pilcher & Organ Mesa Obs. \\
\hline 2013 Mar. 15.1 & 97.4 & 20.6 & 3.7 & 0.004 & F. Pilcher & Organ Mesa Obs. \\
\hline 2013 Mar. 17.2 & 97.7 & 20.7 & 3.9 & 0.005 & F. Pilcher & Organ Mesa Obs. \\
\hline 2013 Mar. 26.2 & 99.3 & 21.1 & 3.8 & 0.006 & F. Pilcher & Organ Mesa Obs. \\
\hline 2013 Mar. 26.8 & 99.5 & 21.1 & 2.5 & 0.009 & R. Hirsch & Borowiec \\
\hline 2014 Apr. 18.0 & 201.3 & 4.0 & 4.5 & 0.008 & - & Montsec Obs. \\
\hline 2014 Apr. 19.0 & 201.1 & 4.2 & 4.6 & 0.011 & - & Montsec Obs. \\
\hline 2014 Apr. 24.0 & 200.1 & 5.5 & 4.2 & 0.012 & - & Montsec Obs. \\
\hline 2014 May 15.2 & 196.8 & 12.0 & 4.5 & 0.005 & F. Pilcher & Organ Mesa Obs. \\
\hline 2014 May 16.2 & 196.6 & 12.3 & 4.6 & 0.005 & F. Pilcher & Organ Mesa Obs. \\
\hline 2014 May 23.9 & 196.0 & 14.2 & 2.0 & 0.005 & - & Montsec Obs. \\
\hline 2014 May 27.0 & 195.9 & 14.9 & 2.0 & 0.007 & - & Montsec Obs. \\
\hline 2015 Jun. 18.0 & 279.8 & 5.6 & 4.7 & 0.005 & - & Montsec Obs. \\
\hline 2015 Jun. 19.0 & 279.6 & 5.4 & 5.5 & 0.006 & - & Montsec Obs. \\
\hline 2015 Jun. 21.0 & 279.3 & 5.0 & 5.2 & 0.011 & - & Montsec Obs. \\
\hline 2015 Jun. 27.1 & 278.0 & 4.2 & 6.8 & 0.003 & A. Marciniak & Obs. del Teide \\
\hline 2015 Jun. 28.9 & 277.6 & 4.1 & 1.5 & 0.003 & A. Marciniak & Obs. del Teide \\
\hline 2015 Jul. 18.0 & 273.9 & 7.6 & 4.5 & 0.010 & - & Montsec Obs. \\
\hline 2015 Jul. 26.9 & 272.5 & 9.9 & 1.5 & 0.006 & A. Marciniak & Borowiec \\
\hline 2015 Aug. 03.7 & 271.6 & 11.9 & 4.2 & 0.003 & M. Żejmo & Adiyaman Obs. \\
\hline 2015 Aug. 08.8 & 271.6 & 13.0 & 2.2 & 0.004 & M. Żejmo & Adiyaman Obs. \\
\hline 2016 Aug. 02.0 & 357.6 & 14.1 & 3.4 & 0.008 & K. Żukowski & Borowiec \\
\hline 2016 Aug. 07.9 & 357.1 & 12.9 & 3.0 & 0.008 & A. Marciniak & Borowiec \\
\hline 2016 Aug. 08.9 & 357.0 & 12.6 & 3.0 & 0.005 & K. Żukowski & Borowiec \\
\hline 2016 Aug. 25.0 & 354.8 & 8.7 & 4.9 & 0.006 & K. Żukowski & Borowiec \\
\hline 2016 Aug. 26.0 & 354.6 & 8.5 & 7.2 & 0.003 & A. Marciniak & Borowiec \\
\hline 2016 Aug. 28.8 & 354.1 & 7.8 & 2.5 & 0.002 & R. Hirsch & Borowiec \\
\hline 2016 Sep. 19.9 & 349.6 & 5.9 & $\begin{array}{l}6.5 \\
180.1 \text { total }\end{array}$ & 0.008 & R. Hirsch & Borowiec \\
\hline
\end{tabular}


Table A.1. continued.

\begin{tabular}{|c|c|c|c|c|c|c|}
\hline Date & $\begin{array}{l}\lambda \\
{[\mathrm{deg}]}\end{array}$ & $\begin{array}{l}\text { Phase angle } \\
\text { [deg] }\end{array}$ & $\begin{array}{l}\text { Duration } \\
\text { [hours] }\end{array}$ & $\begin{array}{l}\sigma \\
{[\mathrm{mag}]}\end{array}$ & Observer & Site \\
\hline (487) Venetia & & & & & & \\
\hline 2006 Apr. 29.1 & 236.4 & 7.6 & 2.5 & 0.013 & L. Bernasconi & Obs. des Engarouines \\
\hline 2006 May 10.0 & 234.0 & 5.1 & 5.9 & 0.015 & L. Bernasconi & Obs. des Engarouines \\
\hline 2006 May 11.0 & 233.8 & 5.0 & 5.8 & 0.009 & L. Bernasconi & Obs. des Engarouines \\
\hline 2012 Oct. 29.0 & 62.3 & 11.7 & 7.5 & 0.012 & M. Bronikowska & Borowiec \\
\hline 2012 Nov. 10.2 & 59.8 & 7.5 & 1.0 & 0.009 & $\begin{array}{l}\text { W. Ogłoza, } \\
\text { E. Kosturkiewicz }\end{array}$ & Suhora \\
\hline 2012 Nov. 11.1 & 59.6 & 7.2 & 4.5 & 0.007 & $\begin{array}{l}\text { W. Ogłoza, } \\
\text { E. Kosturkiewicz }\end{array}$ & Suhora \\
\hline 2012 Dec. 28.8 & 51.2 & 17.1 & 7.5 & 0.008 & K. Sobkowiak & Borowiec \\
\hline 2013 Mar. 02.8 & 61.7 & 22.9 & 3.2 & 0.006 & R. Hirsch & Borowiec \\
\hline 2013 Mar. 03.8 & 62.0 & 22.9 & 2.7 & 0.005 & M. Bronikowska & Borowiec \\
\hline 2014 Feb. 05.1 & 174.6 & 13.0 & 7.8 & 0.006 & R. Hirsch & Borowiec \\
\hline 2014 Feb. 06.1 & 174.5 & 12.7 & 2.7 & 0.006 & A. Marciniak & Borowiec \\
\hline 2014 Feb. 23.1 & 171.5 & 7.2 & 3.8 & 0.008 & K. Sobkowiak & Borowiec \\
\hline 2014 Feb. 23.8 & 171.4 & 7.0 & 5.5 & 0.014 & P. Kankiewicz & Kielce \\
\hline 2014 Mar. 09.1 & 168.3 & 4.4 & 5.1 & 0.007 & R. Hirsch & Borowiec \\
\hline 2014 Mar. 10.1 & 168.1 & 4.5 & 2.1 & 0.016 & J. Horbowicz & Borowiec \\
\hline 2014 Mar. 30.0 & 163.8 & 9.6 & 6.6 & 0.004 & $\begin{array}{l}\text { W. Ogłoza, } \\
\text { E. Kosturkiewicz }\end{array}$ & Suhora \\
\hline 2014 Apr. 11.9 & 161.9 & 13.6 & 5.5 & 0.003 & $\begin{array}{l}\text { M. Siwak, } \\
\text { E. Kosturkiewicz }\end{array}$ & Suhora \\
\hline 2014 Apr. 12.9 & 161.8 & 13.8 & 4.8 & 0.006 & M. Siwak, E. Kosturkiewicz & Suhora \\
\hline 2014 May 21.9 & 162.6 & 20.2 & 3.2 & 0.006 & R. Hirsch & Borowiec \\
\hline 2015 May 08.0 & 263.6 & 12.5 & 3.2 & 0.005 & W. Ogłoza & Suhora \\
\hline 2015 May 10.4 & 263.3 & 11.8 & 2.3 & 0.004 & K. Kamiński & Winer \\
\hline 2015 May 19.0 & 262.0 & 9.0 & 2.4 & 0.002 & M. Żejmo & Adiyaman \\
\hline 2015 May 31.0 & 259.6 & 5.1 & 4.7 & 0.004 & - & Montsec \\
\hline 2015 Jun. 13.9 & 256.4 & 4.0 & 4.0 & 0.004 & M. Żejmo & Adiyaman \\
\hline 2015 Jun. 15.3 & 256.1 & 4.3 & 6.2 & 0.003 & F. Pilcher & Organ Mesa Obs. \\
\hline 2015 Jun. 17.3 & 255.7 & 4.9 & 3.5 & 0.003 & F. Pilcher & Organ Mesa Obs. \\
\hline 2015 Jun. 18.0 & 255.5 & 5.1 & $\begin{array}{l}5.3 \\
119.3 \text { total }\end{array}$ & 0.005 & - & Montsec \\
\hline
\end{tabular}




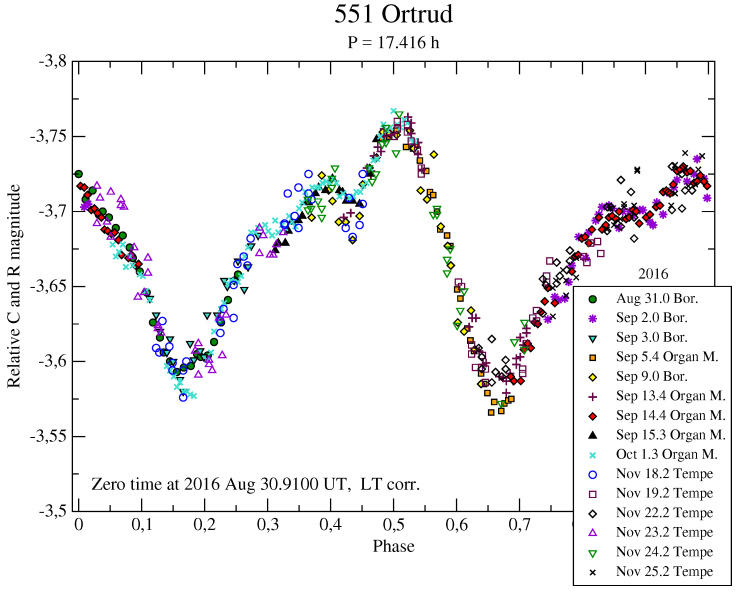

Fig. A.1. Composite lightcurve of (551) Ortrud in the year 2016.

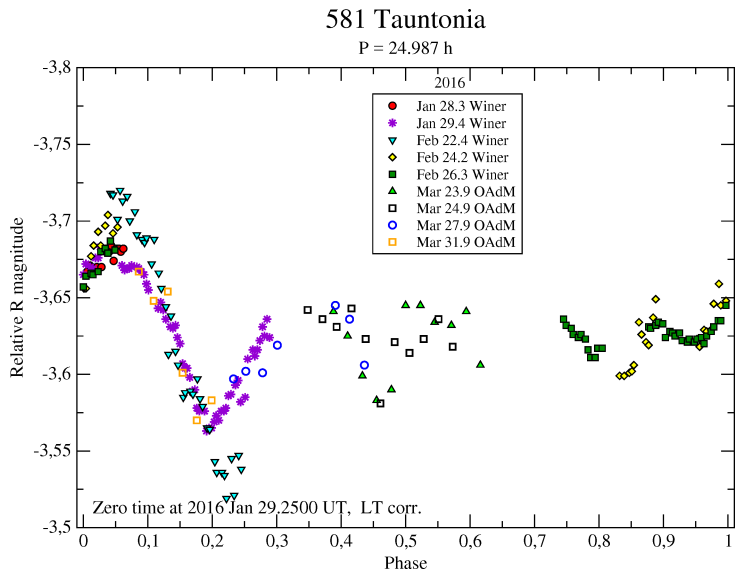

Fig. A.2. Composite lightcurve of (581) Tauntonia in 2016.

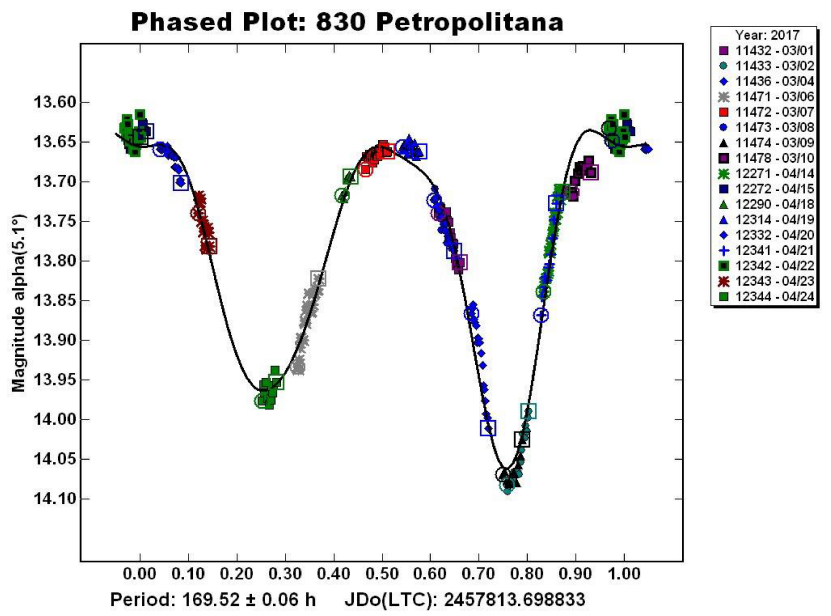

Fig. A.3. Calibrated composite lightcurve of (830) Petropolitana in 2017.

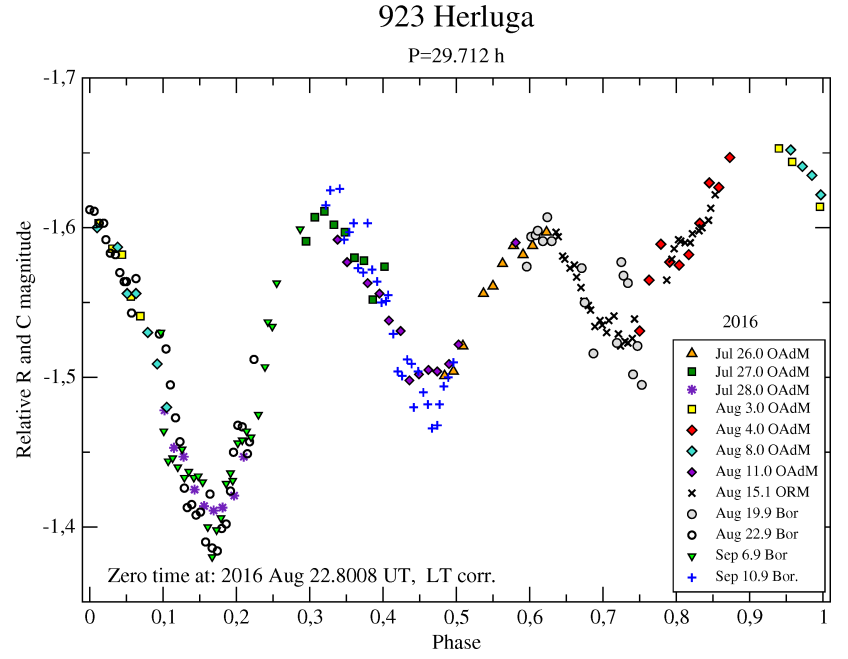

Fig. A.4. Composite lightcurve of (923) Herluga in 2016.
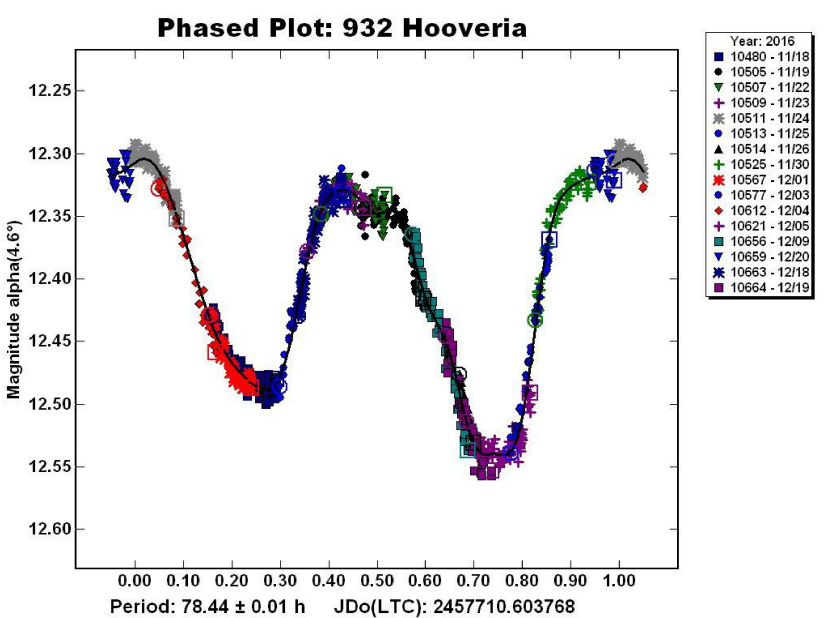

Fig. A.5. Calibrated composite lightcurve of (932) Hooveria in 2016.

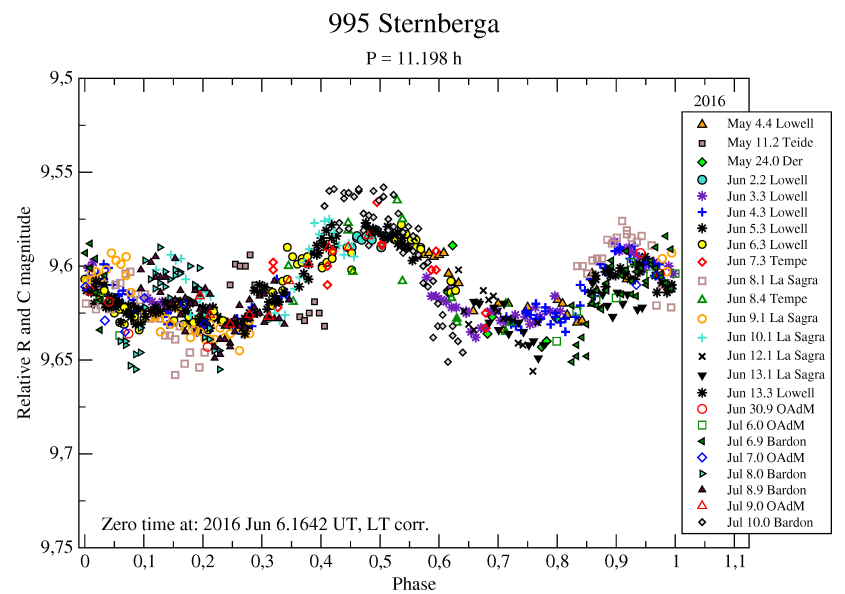

Fig. A.6. Composite lightcurve of (995) Sternberga in 2016. 

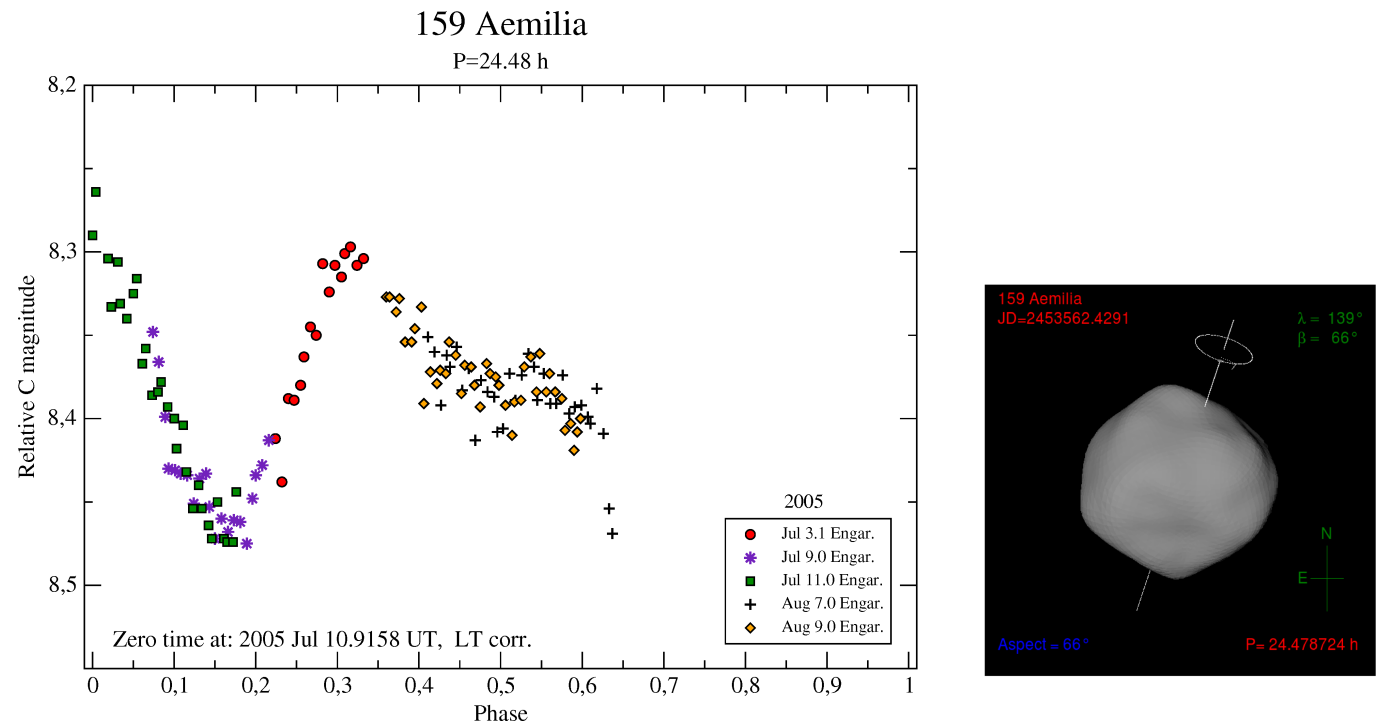

Fig. A.7. Composite lightcurve of (159) Aemilia in the year 2005 with the orientation of SAGE model 1 for the zero phase.
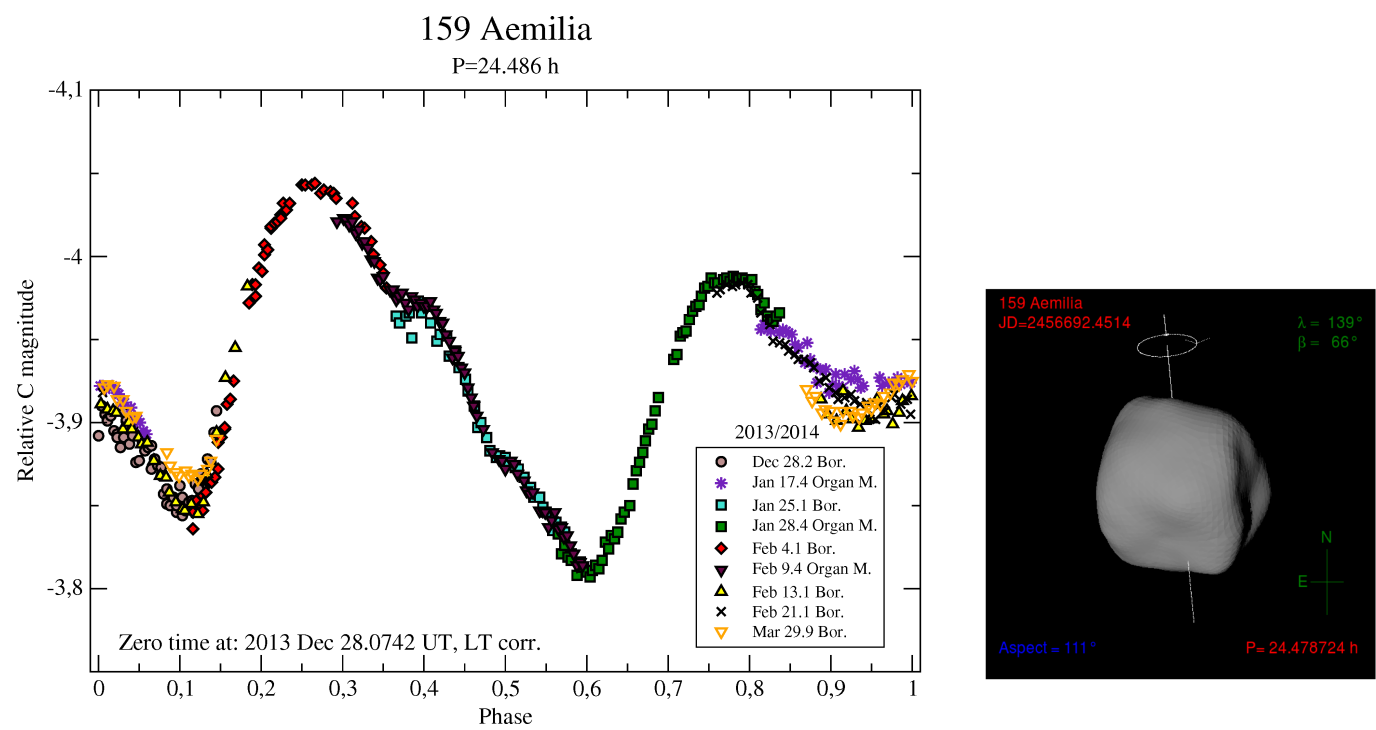

Fig. A.8. Composite lightcurve of (159) Aemilia in the years 2013-2014 with the orientation of SAGE model 1 for the zero phase.
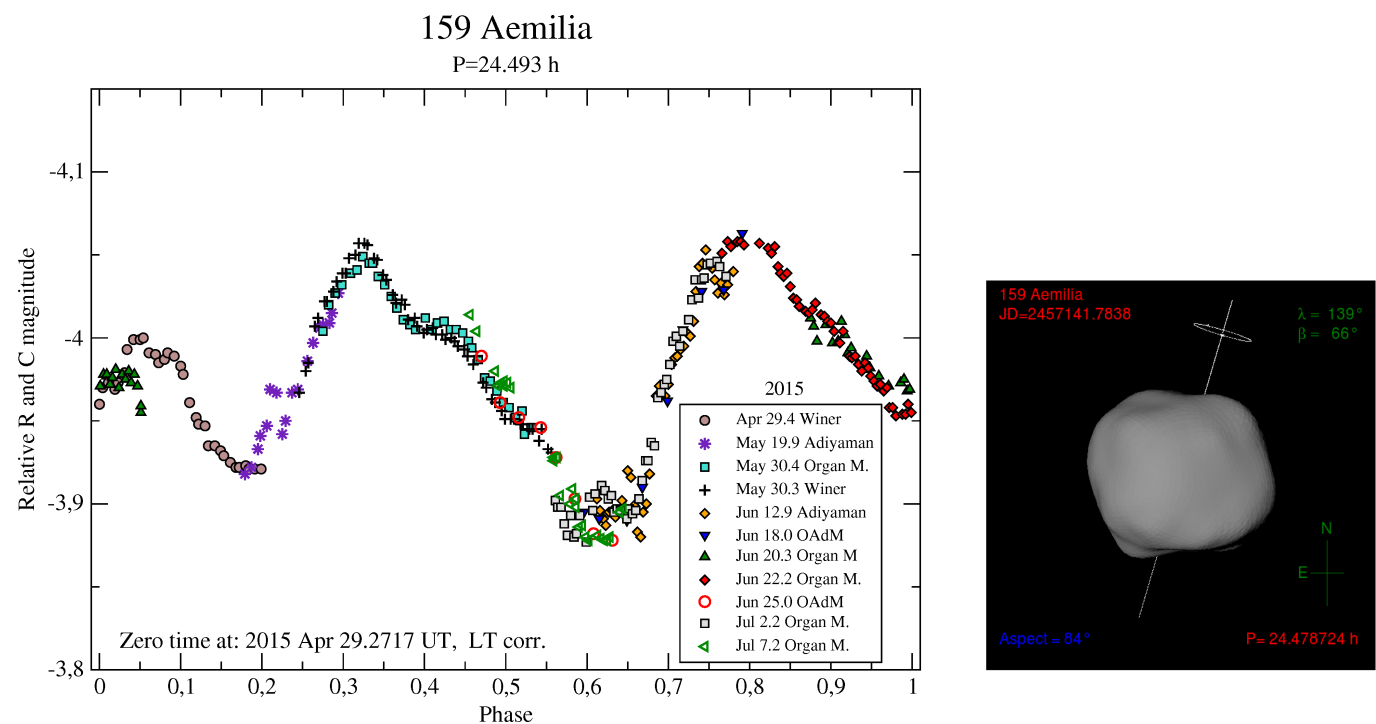

Fig. A.9. Composite lightcurve of (159) Aemilia in the year 2015 with the orientation of SAGE model 1 for the zero phase. 
227 Philosophia
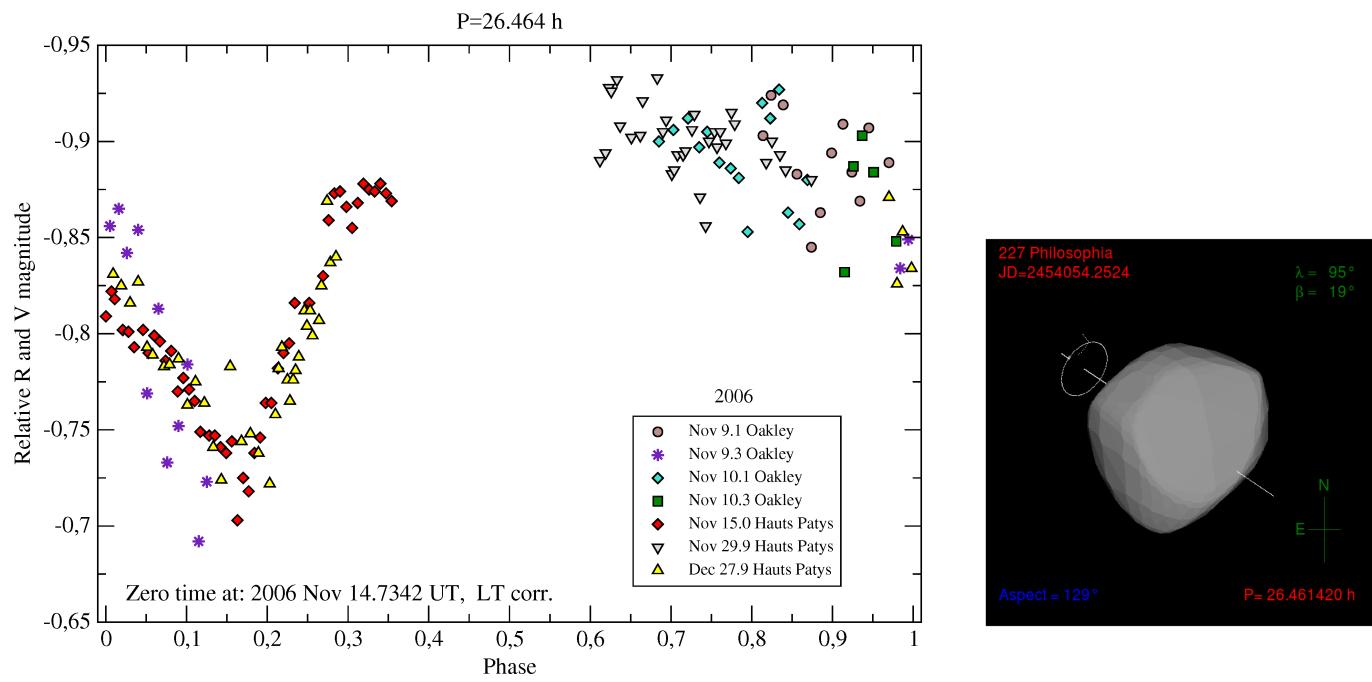

Fig. A.10. Composite lightcurve of (227) Philosophia in the year 2006 with the orientation of convex model 1 for the zero phase.
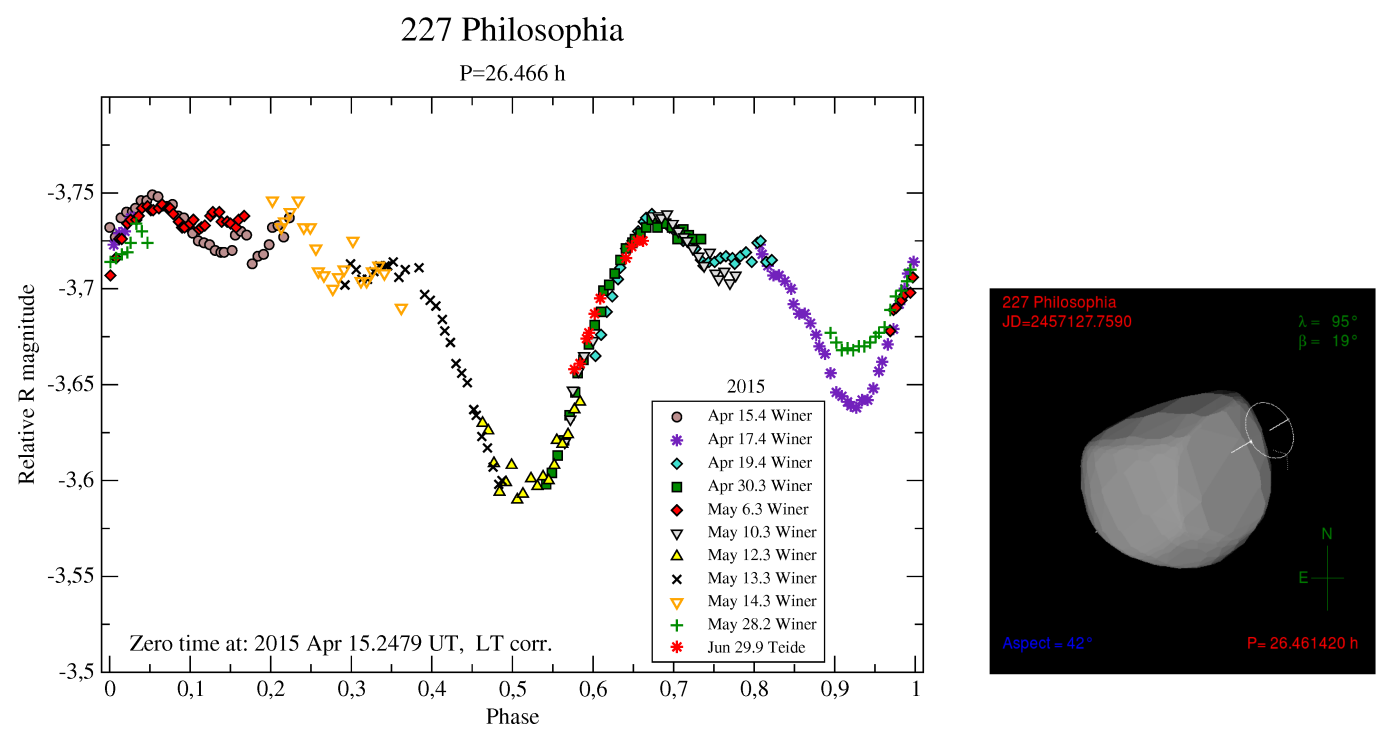

Fig. A.11. Composite lightcurve of (227) Philosophia in the year 2015 with the orientation of convex model 1 for the zero phase.

(227) Philosophia
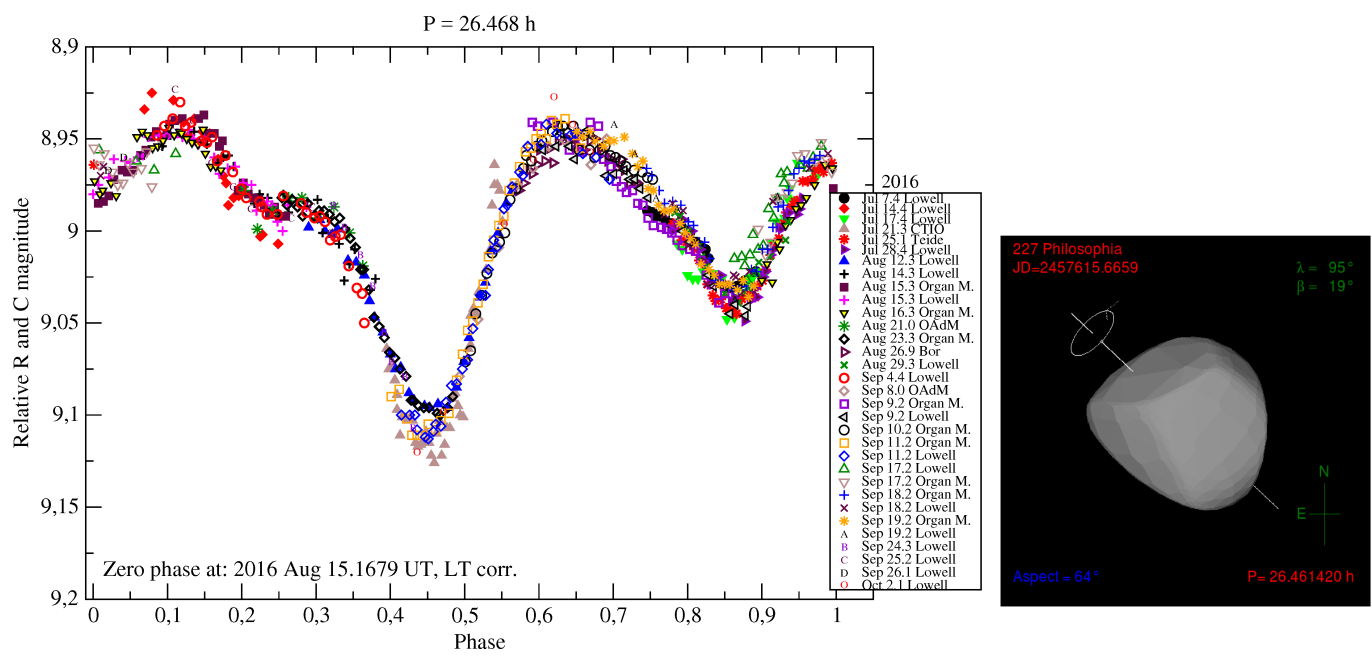

Fig. A.12. Composite lightcurve of (227) Philosophia in the year 2016 with the orientation of convex model 1 for the zero phase. 

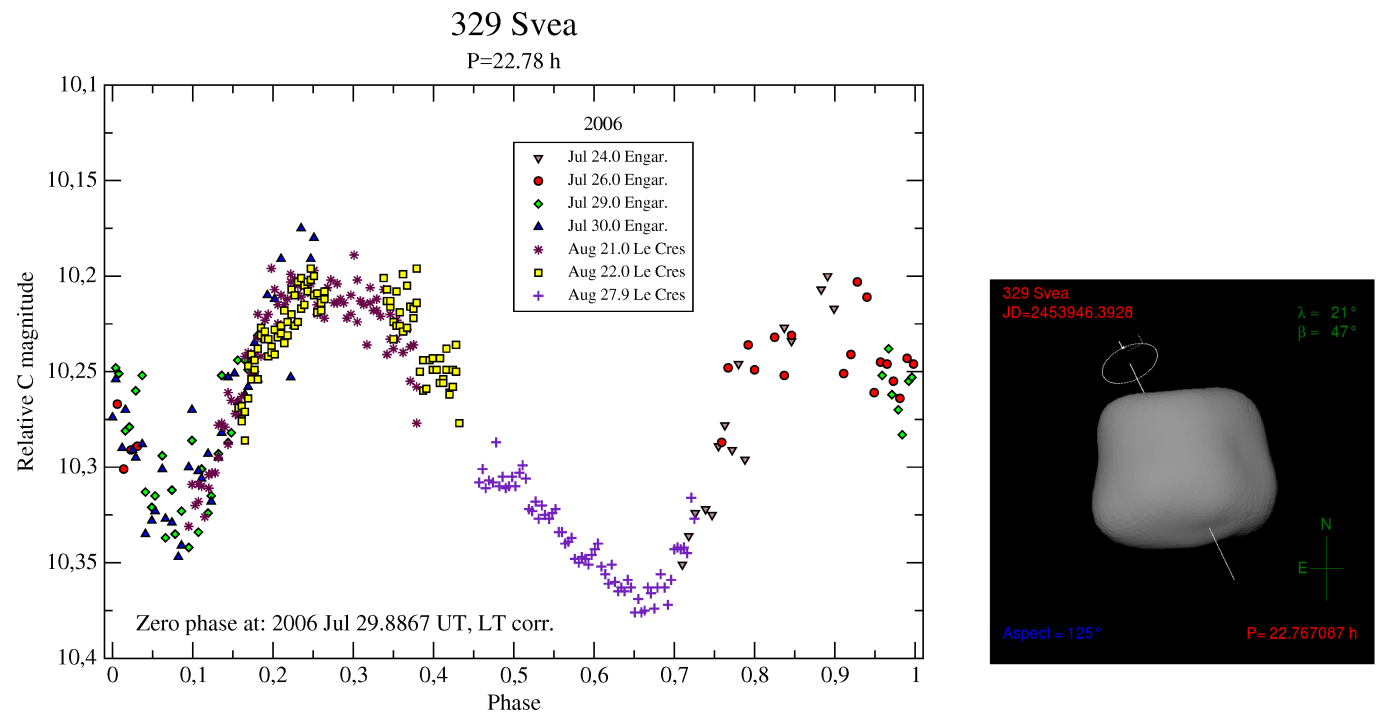

Fig. A.13. Composite lightcurve of (329) Svea in the year 2006 with the orientation of SAGE model 1 for the zero phase.
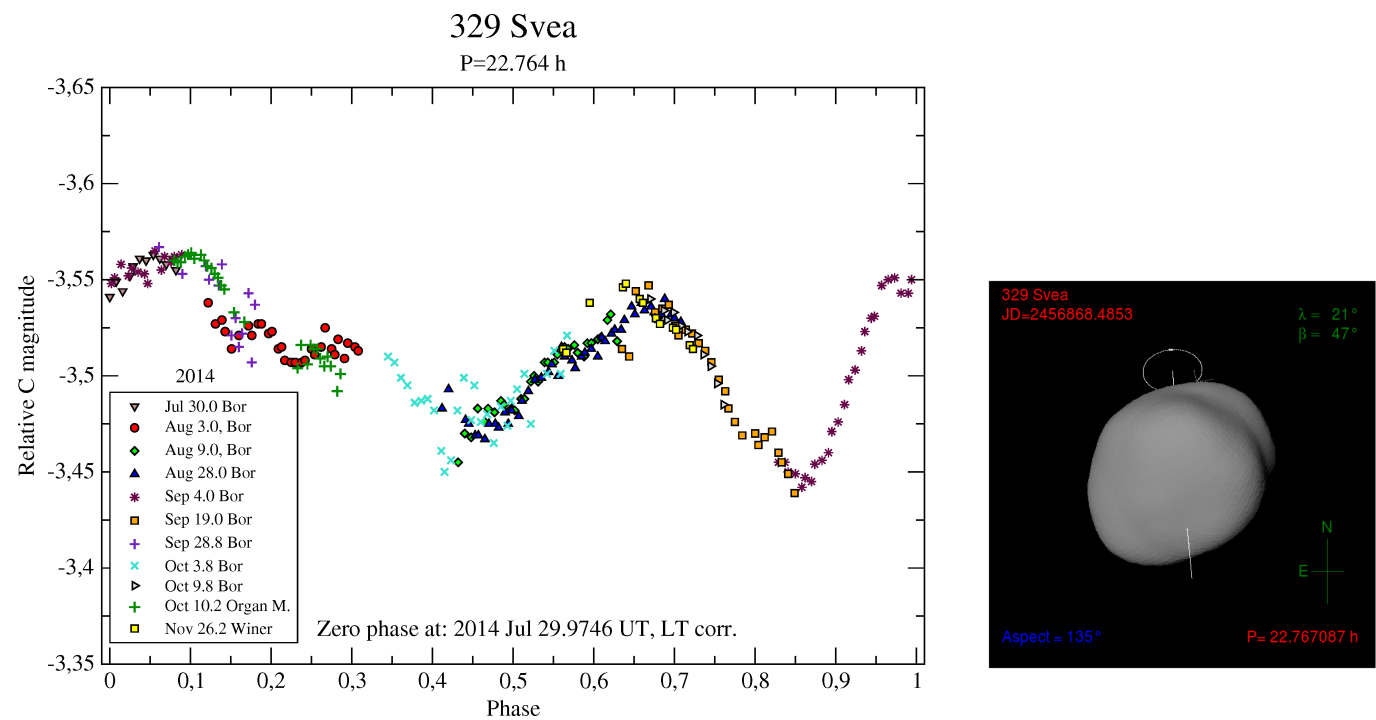

Fig. A.14. Composite lightcurve of (329) Svea in the year 2014 with the orientation of SAGE model 1 for the zero phase.
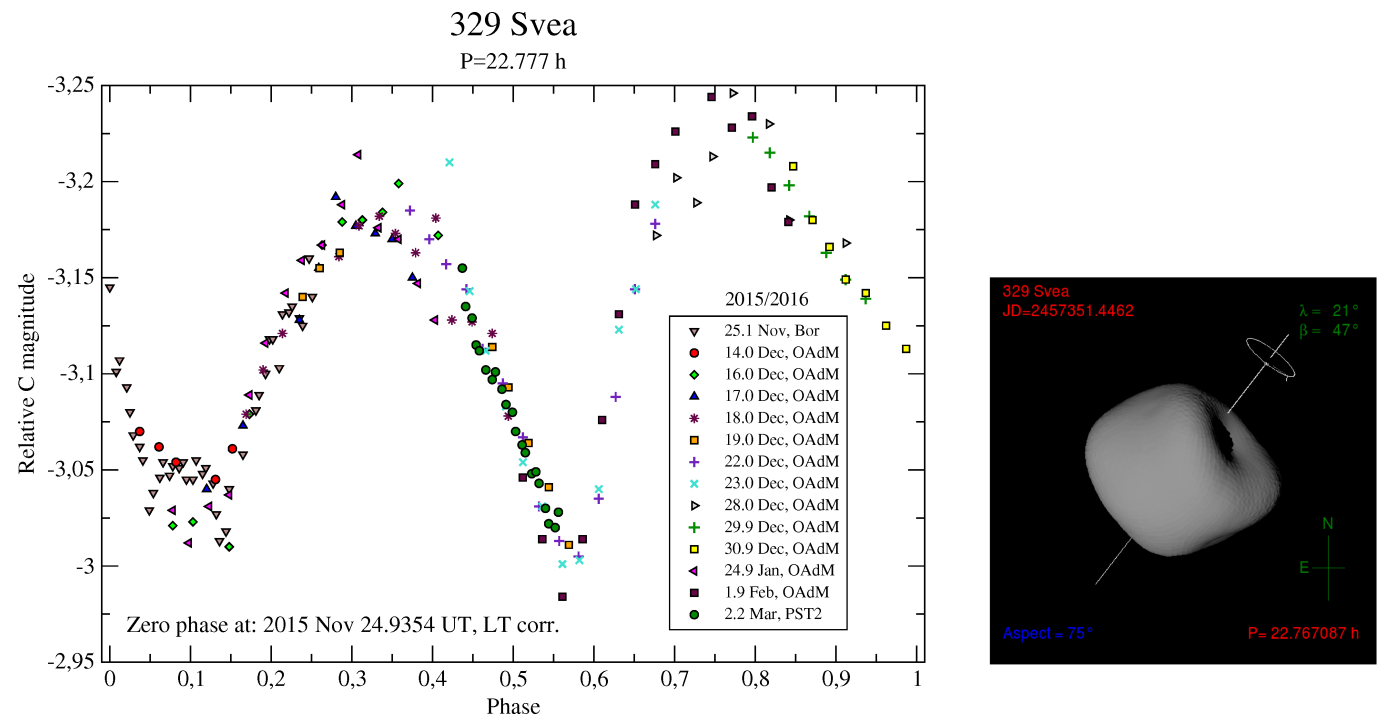

Fig. A.15. Composite lightcurve of (329) Svea in the years 2015-2016 with the orientation of SAGE model 1 for the zero phase. 

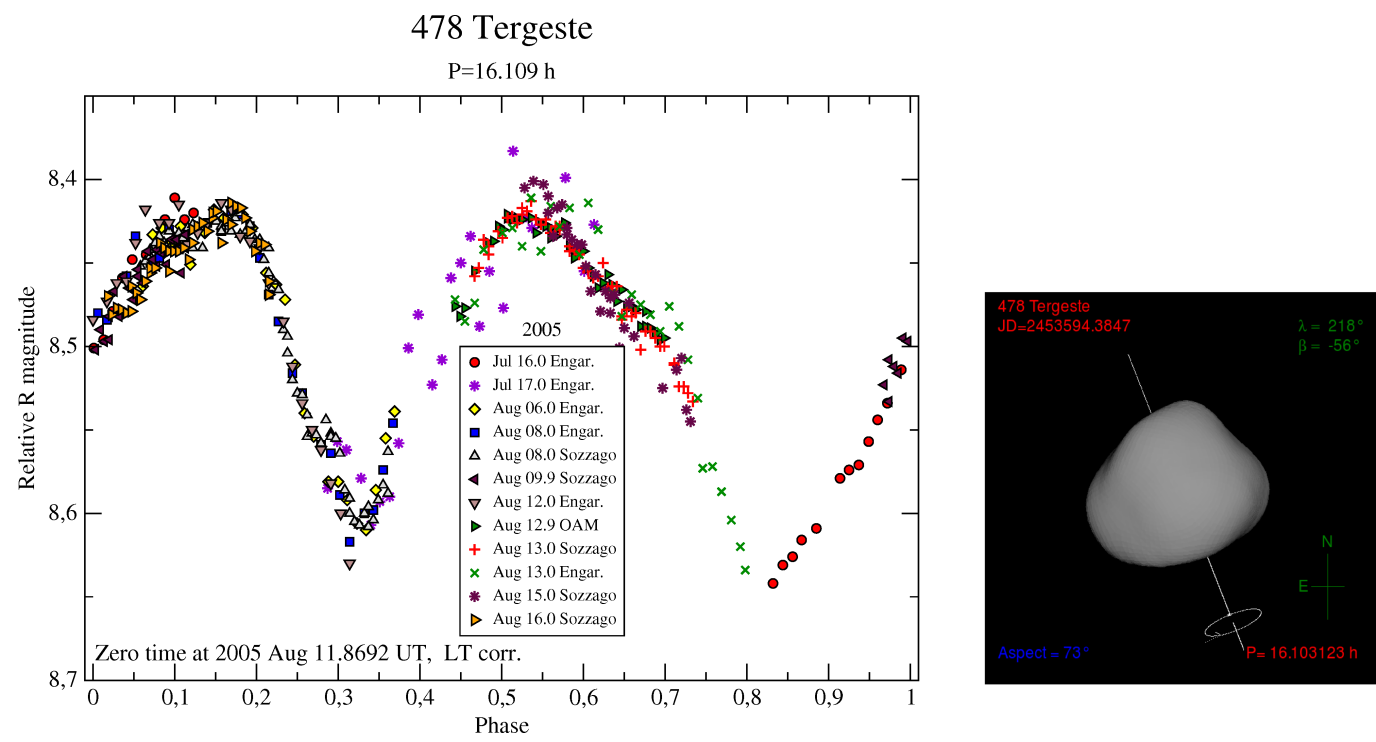

Fig. A.16. Composite lightcurve of (478) Tergeste in the year 2005 with the orientation of SAGE model 2 for the zero phase.

\section{Tergeste}
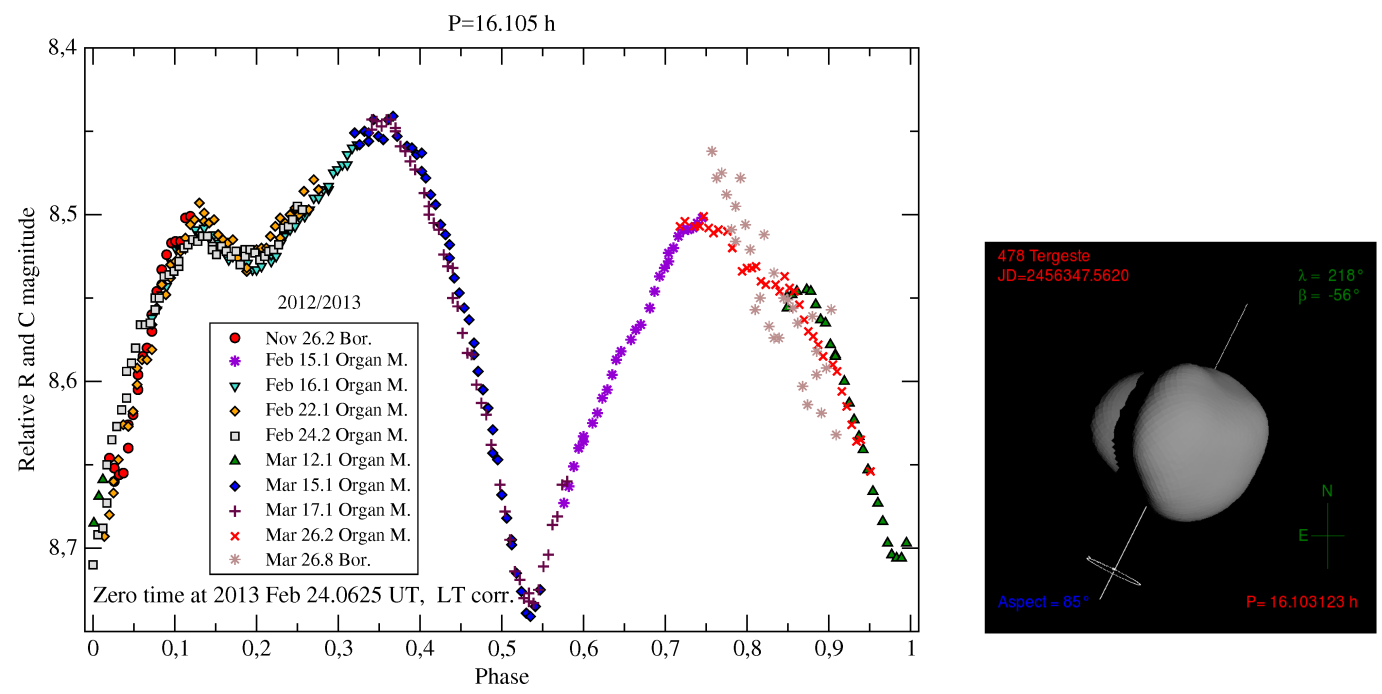

Fig. A.17. Composite lightcurve of (478) Tergeste in the years 2012-2013 with the orientation of SAGE model 2 for the zero phase.
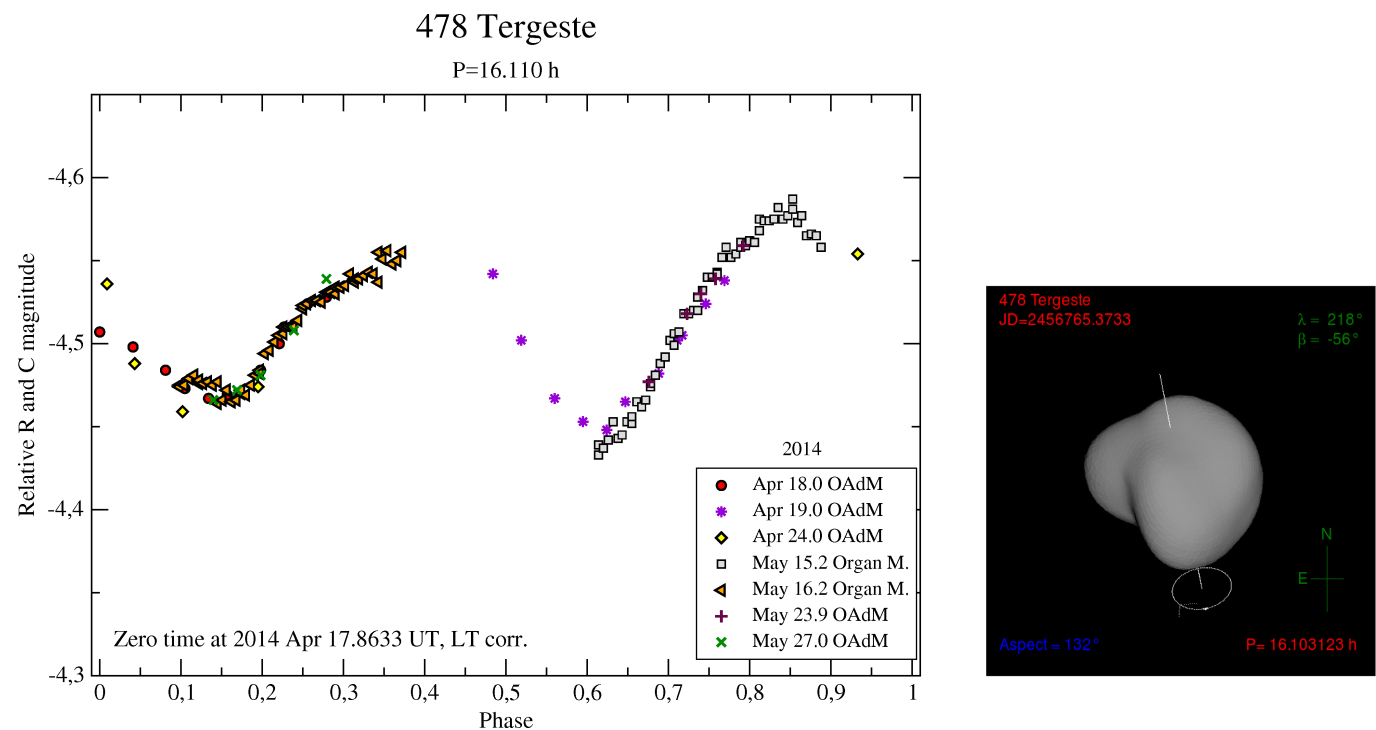

Fig. A.18. Composite lightcurve of (478) Tergeste in the year 2014 with the orientation of SAGE model 2 for the zero phase. 
A. Marciniak et al.: Long-period and low-amplitude asteroids
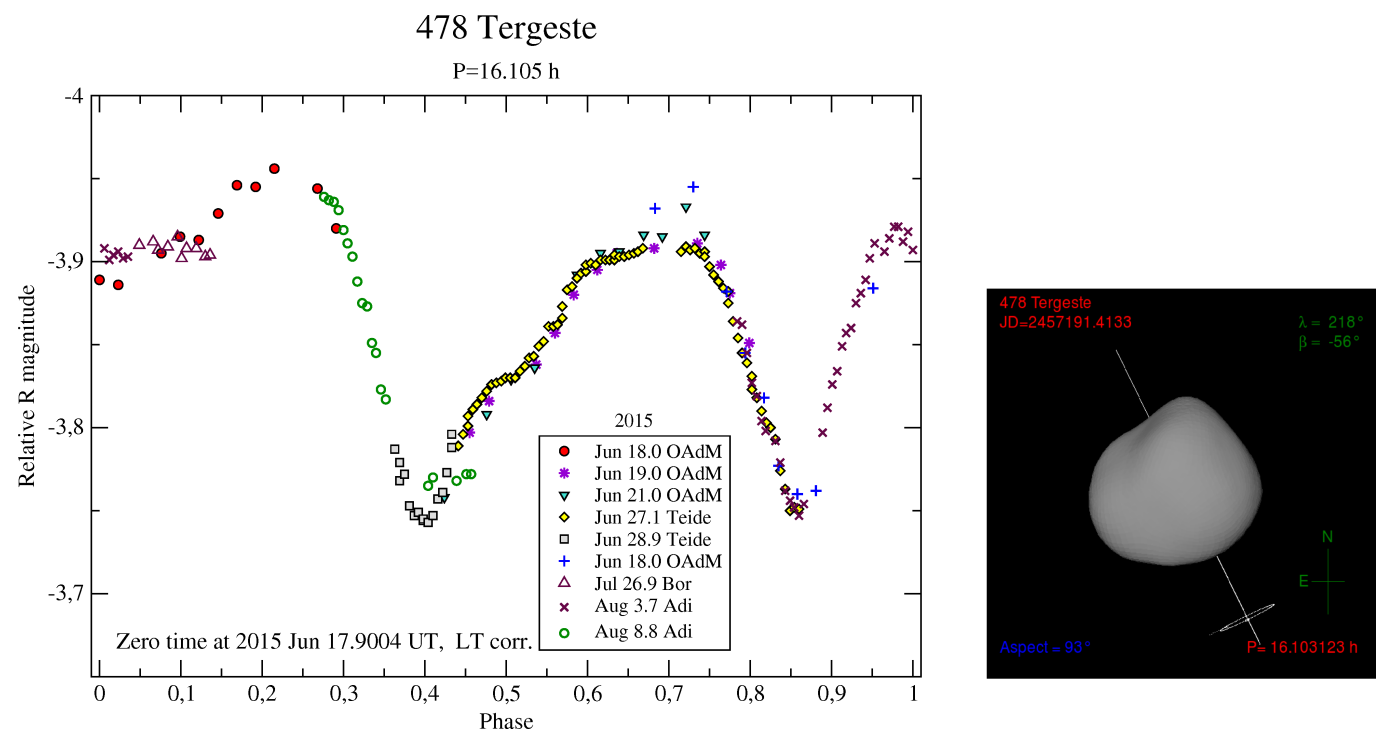

Fig. A.19. Composite lightcurve of (478) Tergeste in the year 2015 with the orientation of SAGE model 2 for the zero phase.
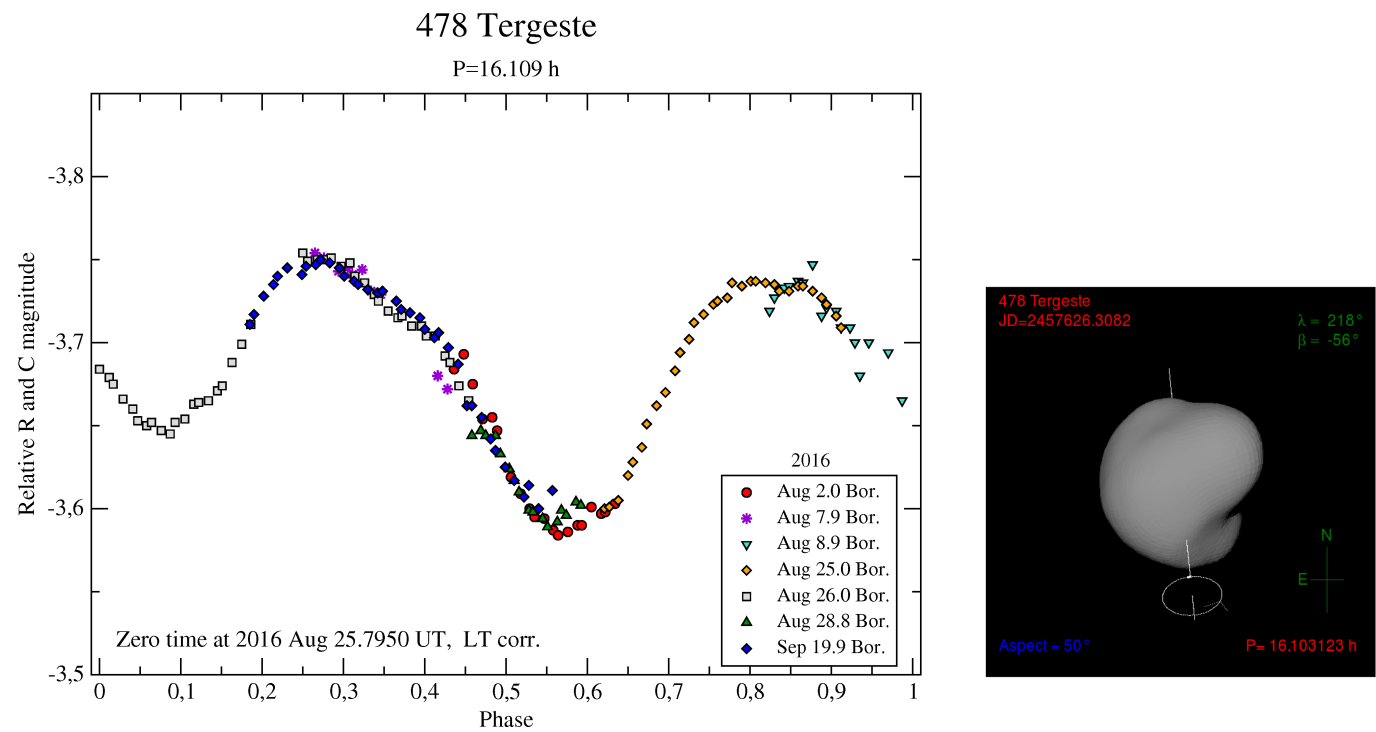

Fig. A.20. Composite lightcurve of (478) Tergeste in the year 2016 with the orientation of SAGE model 2 for the zero phase.
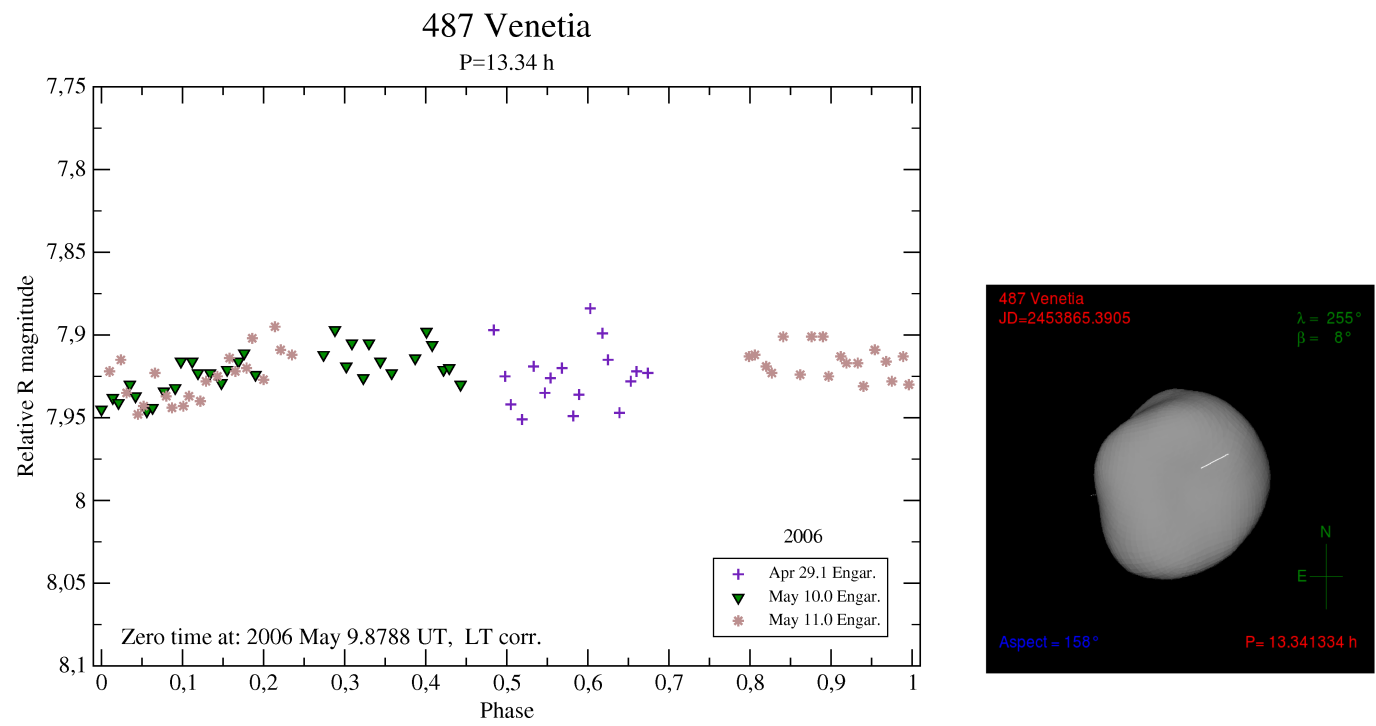

Fig. A.21. Composite lightcurve of (487) Venetia in the year 2006 with the orientation of SAGE model 2 for the zero phase. 


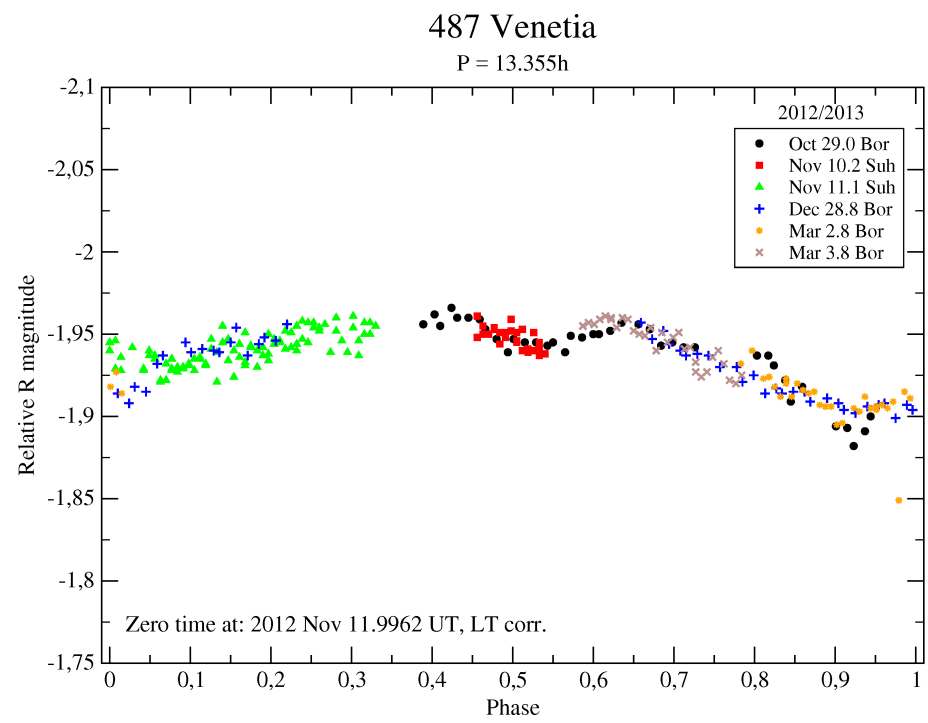

Fig. A.22. Composite lightcurve of (487) Venetia in the years 2012-2013 with the orientation of SAGE model 2 for the zero phase.
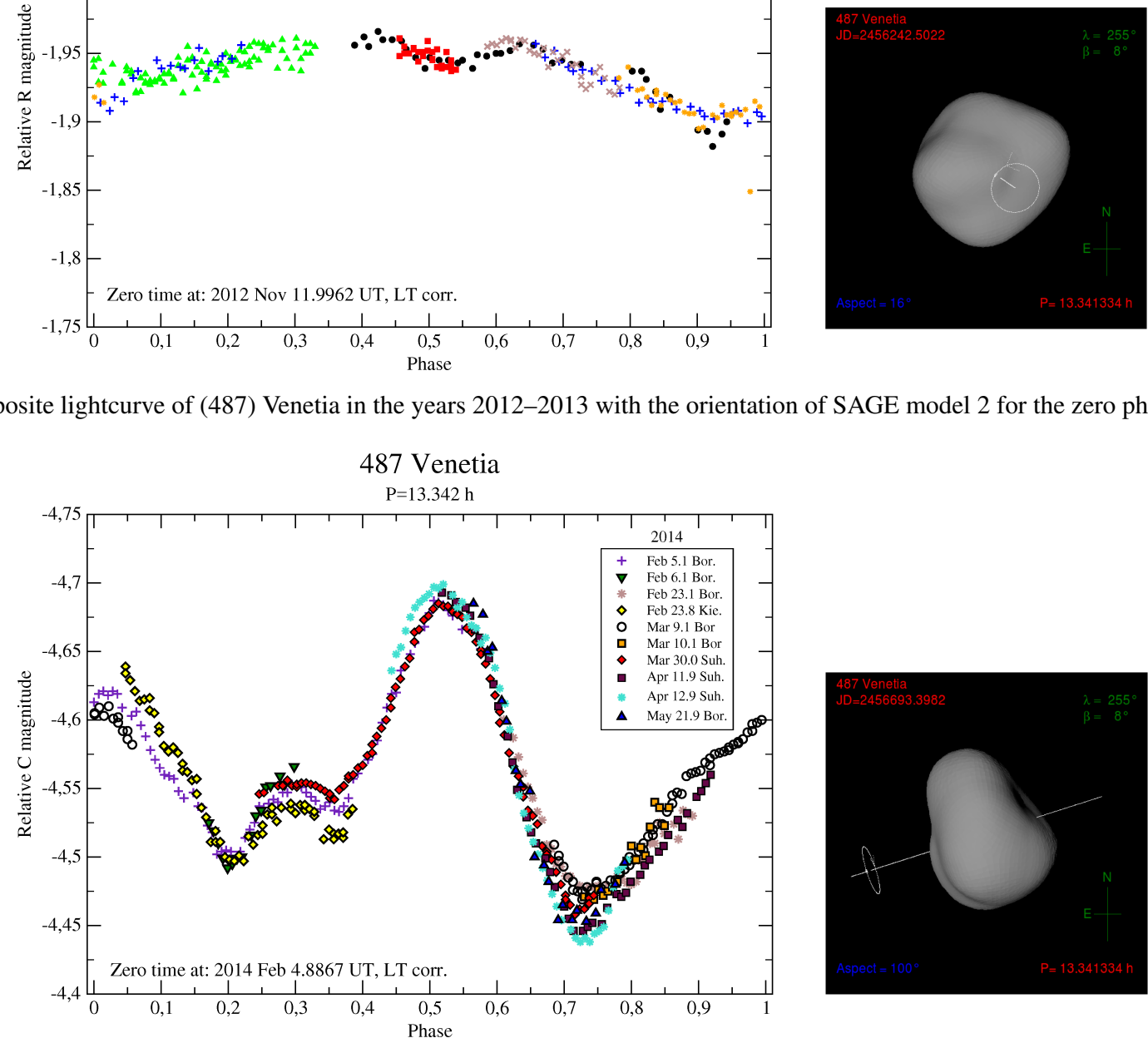

Fig. A.23. Composite lightcurve of (487) Venetia in the year 2014 with the orientation of SAGE model 2 for the zero phase.
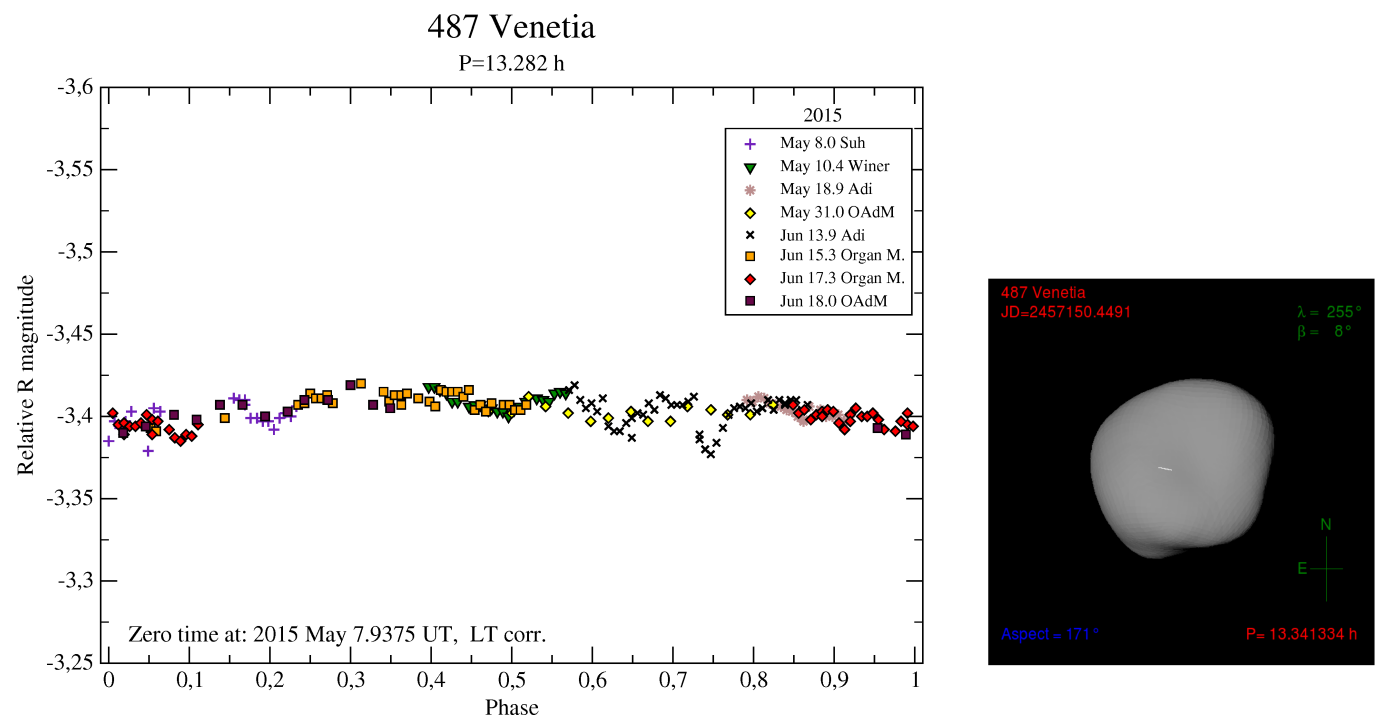

Fig. A.24. Composite lightcurve of (487) Venetia in the year 2015 with the orientation of SAGE model 2 for the zero phase. 


\section{Appendix B}

List of stellar occultation observers.

(159) Aemilia (2009-05-02), USA

S. Meesner (Northfield, Minnesota)

S. Conard (Gamber, Maryland)

B. Koch (Faribault, Minnesota)

A. Scheck (Laurel, Maryland)

(329) Svea (2011-12-28), Japan

H. Tomioka (Hitachi city, Ibaraki Prefecture)

H. Takashima (Kashiwa, Chiba)

K. Kitazato (Musashino, Tokyo)

Y. Watanabe (Inabe, Mie)

S. Ida (Higashiomi, Shiga)

M. Ishida (Moriyama, Shiga)

M. Owada (Hamamatsu, Shizuoka)

K. Kasazumi (Takatsuki, Osaka)

S. Okamoto (Tsuyama, Okayama)

N. Tatsumi (Akaiwa, Okayama)

Hironaka and Miyamaoto (Hiroshima University Observatory,

Hiroshima)

(329) Svea (2013-03-07), USA

P. Maley (5 sites, Forida)

D. Liles (Florida)

A. Cruz (Glen St. Mary, Florida)

E. Gray (Macclenny, Florida)

J. Brueggemann (Florida)

C. McDougal (Tampa, Florida)

T. Campbel (3 sites, Florida) 